\title{
The Pierre Auger Cosmic Ray Observatory
}

\author{
The Pierre Auger Collaboration ${ }^{1}$ \\ Pierre Auger Collaboration, Av. San Martín Norte 306, 5613 Malargüe, Mendoza, Argentina
}

\section{A R T I C L E I N F O}

\section{Article history:}

Received 30 January 2015

Received in revised form

29 June 2015

Accepted 30 June 2015

Available online 8 July 2015

Keywords:

Pierre Auger Observatory

High energy cosmic rays

Hybrid observatory

Water Cherenkov detectors

Air fluorescence detectors

\begin{abstract}
A B S T R A C T
The Pierre Auger Observatory, located on a vast, high plain in western Argentina, is the world's largest cosmic ray observatory. The objectives of the Observatory are to probe the origin and characteristics of cosmic rays above $10^{17} \mathrm{eV}$ and to study the interactions of these, the most energetic particles observed in nature. The Auger design features an array of 1660 water Cherenkov particle detector stations spread over $3000 \mathrm{~km}^{2}$ overlooked by 24 air fluorescence telescopes. In addition, three high elevation fluorescence telescopes overlook a $23.5 \mathrm{~km}^{2}$, 61-detector infilled array with $750 \mathrm{~m}$ spacing. The Observatory has been in successful operation since completion in 2008 and has recorded data from an exposure exceeding $40,000 \mathrm{~km}^{2} \mathrm{sr} \mathrm{yr}$. This paper describes the design and performance of the detectors, related subsystems and infrastructure that make up the Observatory.
\end{abstract}

(c) 2015 The Authors. Published by Elsevier B.V. This is an open access article under the CC BY-NC-ND license (http://creativecommons.org/licenses/by-nc-nd/4.0/).

\section{Introduction}

The origin of high energy cosmic rays has been a mystery since the discovery of extensive air showers in the late 1930s [1-5]. In 1962, John Linsley recorded a cosmic ray event with an energy of $10^{20} \mathrm{eV}$ [6]. Subsequent work found more events near and above $10^{20} \mathrm{eV}[7-10]$. The Pierre Auger Observatory was proposed to discover and understand the source or sources of cosmic rays with the highest energies.

A unique partnership of 18 countries, the Pierre Auger Collaboration came together to pursue this science. Construction of the Pierre Auger Observatory was started in 2002 and completed in 2008. The purpose of this paper is to review the design and performance of the detector systems and associated infrastructure that constitute the Observatory.

To achieve the scientific goals, the Collaboration designed the Pierre Auger Observatory for a high statistics study of cosmic rays at the highest energies. Measured properties of the air showers determine the energy and arrival direction of each cosmic ray. These properties also provide a statistical determination of the distribution of primary masses (cosmic ray composition). The Pierre Auger Observatory in the Province of Mendoza, Argentina, has been taking data since 2004 , adding detectors as they became active until completion in 2008.

The Observatory is a hybrid detector, a combination of a large surface detector (SD) and a fluorescence detector (FD). The SD is

\footnotetext{
${ }^{1}$ See Appendix A for further details on the collaboration.

E-mail address: auger_spokespersons@fnal.gov

URL: http://www.auger.org
}

composed of a baseline array, comprising 1660 water Cherenkov stations placed in a triangular grid with nearest neighbors separated by $1500 \mathrm{~m}$, and a smaller array (stations separated by $750 \mathrm{~m}$ ). The surface array is spread over an area of $\sim 3000 \mathrm{~km}^{2}$, and is depicted in Fig. 1. Figure 2 shows examples of FD (left) and SD (right) detector elements. This area is generally flat, with detectors located at altitudes between $1340 \mathrm{~m}$ and $1610 \mathrm{~m}$. The mean altitude is $\sim 1400 \mathrm{~m}$, corresponding to an atmospheric overburden of $\sim 875 \mathrm{~g} \mathrm{~cm}^{-2}$. The array is located between latitudes $35.0^{\circ}$ and $35.3^{\circ} \mathrm{S}$ and between longitudes $69.0^{\circ}$ and $69.4^{\circ} \mathrm{W}$.

As an aid to the reader we provide here a list of acronyms used throughout this paper. ${ }^{2}$

\subsection{Highlights of science results}

The Pierre Auger Observatory has made key measurements of the highest energy cosmic rays. Cosmic ray showers with zenith angle $<60^{\circ}$ are defined as vertical showers, while those with $60^{\circ}<$ zenith angle $<80^{\circ}$ are defined as horizontal showers. The energy spectrum is measured with unprecedented precision using

\footnotetext{
${ }^{2}$ AERA, Auger Engineering Radio Array; AMIGA, Auger Muon and Infilled Ground Array; APF, aerosol phase function; ARQ Automatic Repeat Request; BLS, Balloon Launching Station; CDAS, central data acquisition system; CIC, Constant Intensity Cut; CLF, Central Laser Facility; EAS, extensive air shower; ELVES: (defined in Section 14.2); FADC, flash analog-to-digital convertor; FD, fluorescence detector: FRAM, Photometric Robotic Atmospheric Monitor; GDAS, Global Data Assimilation System; HAM, Horizontal Attenuation Monitor; HEAT, High-Elevation Auger Telescopes; LDF, lateral distribution function; MD, muon detector; PLD, programmable logic device; PMT, photomultiplier tube; SCS, slow control system; SD, surface detector; SDP, shower-detector plane; TDMA, time division multiple access; ToT, time over threshold; VEM, vertical equivalent muon; XLF, eXtreme Laser Facility.
} 


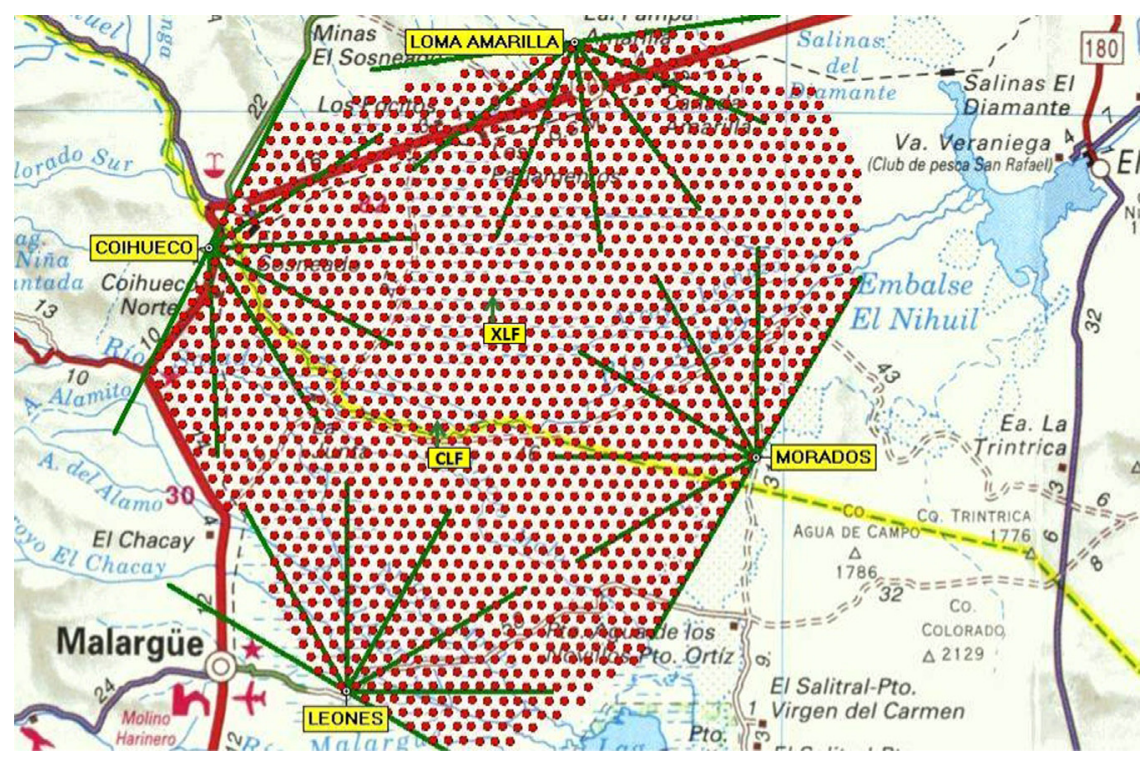

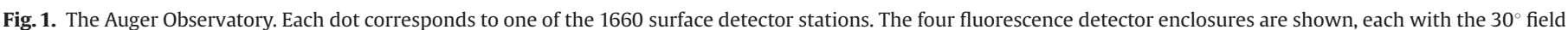
of view of its six telescopes. Also shown are the two laser facilities, CLF and XLF, near the Observatory center.
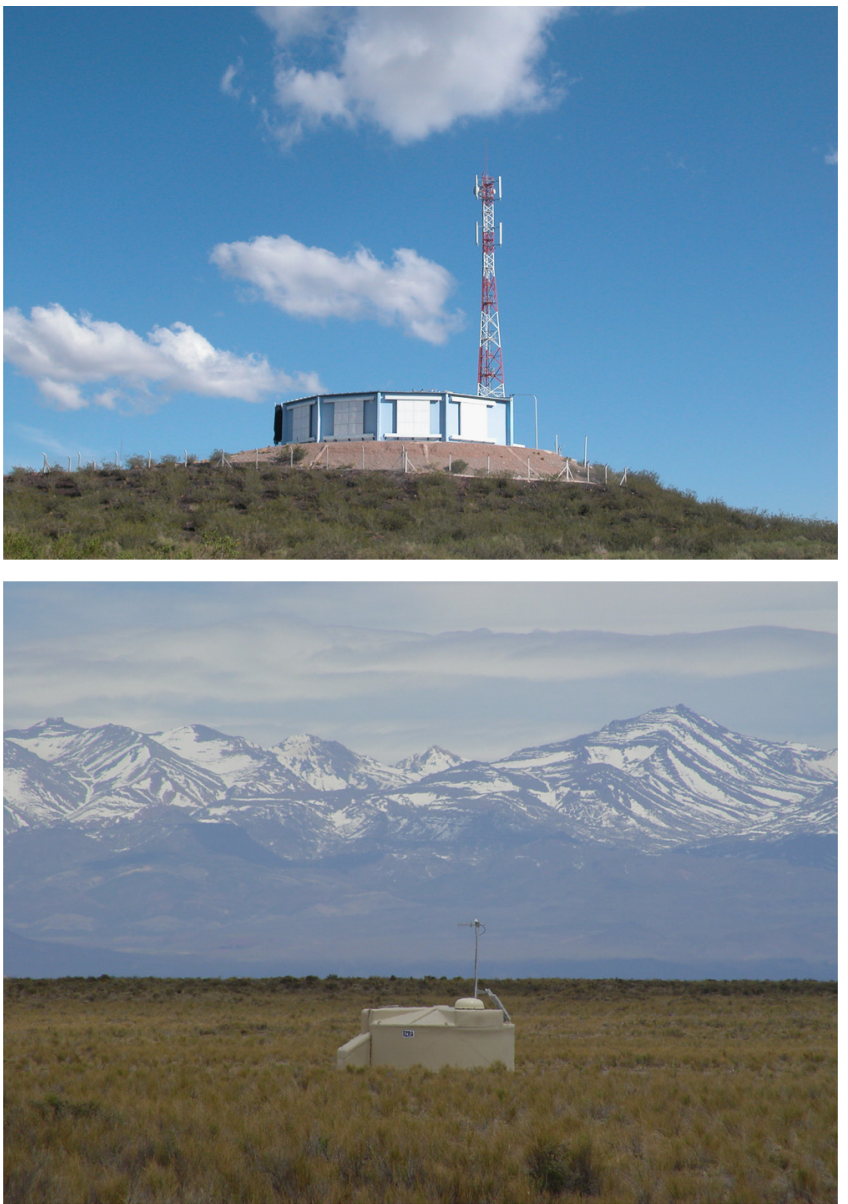

Fig. 2. The fluorescence detector enclosure Los Leones (top) and a surface detector station (bottom)

four datasets: hybrid (events measured simultaneously by the SD and FD), SD $750 \mathrm{~m}$ array, SD baseline vertical, and SD baseline horizontal [11,12]. Thanks to the high statistics of the SD data, a first harmonic analysis was performed in different energy ranges starting from $2.5 \times 10^{17} \mathrm{eV}$ in a search for dipolar modulations in right ascension $[13,14]$. The upper limits in the dipole amplitude impose stringent constraints on astrophysical models $[13,15,16]$.

The Auger data provide evidence for a weak correlation between arrival directions of cosmic rays above $55 \mathrm{EeV}$ and the positions of AGNs with $z<0.018$ in the VCV catalog $[17,18]$. The Collaboration also has performed the measurement of the protonair cross-section at $57 \mathrm{TeV}$ [19] that favors a moderately slow rise of the cross-section towards higher energies, and inferred the proton-proton cross-section, whose value is within one sigma of the best extrapolation from the recent LHC data points [20]. The composition measurements could be interpreted as an evolution from light to heavier nuclei if current hadronic interaction models describe well the air shower physics [21-24].

Upper limits have been obtained on the photon flux integrated above an energy threshold which impose stringent limits for topdown models [25,26]. Also, competitive neutrino limits were published [27-29], as well as searches for Galactic neutron signals $[30,31]$.

\subsection{Observatory design}

Design targets for the surface detector array included 100\% duty cycle, a well-defined aperture independent of energy above $10^{18.5} \mathrm{eV}$, measurement of the time structure of the signals of the shower particles, sensitivity to showers arriving at large zenith angles, selfcontained detector stations and in situ calibration of detector stations by cosmic ray muons. The fluorescence detector design required that every event above $10^{19} \mathrm{eV}$ arriving within the FD on-time should be recorded by at least one fluorescence telescope camera, direct measurement of the longitudinal development profile and timing synchronization for simultaneous measurement of showers with the surface detector array [32].

Each water Cherenkov surface detector is self-powered and communicates with the central data acquisition system using wireless technology. Air fluorescence telescopes record air shower development in the atmosphere above the surface array on dark moonless nights. There are four air fluorescence sites on the perimeter of the array, each with six telescopes.

An essential feature of this Auger hybrid design is the capability of observing air showers simultaneously by two different but complementary techniques. The SD operates continuously, measuring the particle densities as the shower strikes the ground just beyond its 
maximum development. On dark nights, the FD records the development of the air shower via the amount of nitrogen fluorescence produced along its path. Since the intensity of fluorescence light is proportional to the energy dissipated by the shower, integrating the intensity of light produced along the shower axis yields a nearly calorimetric measurement of the cosmic ray energy. Using the observation of hybrid events, this energy calibration can then be transferred to the surface array with its $100 \%$ duty factor and large event gathering power. Moreover, independent measurements by the surface array and the fluorescence detectors alone have limitations that can be overcome by comparing their measurements in the set of showers measured by both. The hybrid dataset provides a thorough understanding of the capabilities and the systematic uncertainties of both components.

The FD always operates in conjunction with the SD. Its primary purpose is to measure the longitudinal profile of showers recorded by the SD whenever it is dark and clear enough to make reliable measurements of atmospheric fluorescence from air showers. The integral of the longitudinal profile is used to determine the shower energy, and the rate of shower development is indicative of the primary particle's mass. The hybrid detector has better angular resolution than the surface array by itself.

Subsequent to the completion of construction, two significant enhancements have been incorporated into the baseline detectors that significantly extend the Observatory's science capability. The HEAT (High Elevation Auger Telescopes) fluorescence detectors together with a $750 \mathrm{~m}$ array, part of AMIGA (Auger Muon and Infilled Ground Array) extend the sensitivity down to $10^{17} \mathrm{eV}$, in keeping with the hybrid detection strategy of the original Observatory.

\subsection{Background}

The Pierre Auger Observatory was conceived during the International Cosmic Ray Conference in Dublin in 1991 by Jim Cronin of the University of Chicago and Alan Watson of the University of Leeds. It had become clear to them that only the construction of a very large air shower array would yield the statistical power and complete sky coverage necessary to address the question of the origin of the highest energy cosmic rays.

A six-month design workshop was held in 1995 that produced a Design Report [32] with a discussion of the science, a conceptual design and cost estimate. The design report became the basis for funding proposals by the collaborating countries. Subsequent to the workshop a team of scientists evaluated numerous prospective sites in both hemispheres. Preferred sites were selected in the southern and northern hemispheres by the collaboration in 1995 and 1996, respectively. At the direction of the funding agencies, the project was to begin by building the Observatory in the southern hemisphere.

After a period of research and development, the Engineering Array, consisting of 32 prototype surface array detectors and two prototype fluorescence telescopes, was built to validate the design [33]. At the end of 2001, before the end of the scheduled two years, the Engineering Array was able to record and reconstruct air shower events simultaneously by the surface array and the fluorescence detectors. The Engineering Array demonstrated the validity of the design and the performance of all of the detector systems, communications equipment and data acquisition as well as the deployment methodology. The detectors performed better than the design requirements, substantially increasing the physics reach of the Observatory.

Installation of production detectors began in 2002. While the Engineering Array was assembled and deployed almost completely by Auger collaborators, production deployment was accomplished by trained Observatory staff. Scientists monitored the quality of the work and carried out the commissioning of completed detectors. The Observatory started collecting data in January 2004 with 154 active detector stations. The first physics results were presented during the 2005 summer conference season.

Many important results have now been published by the Auger Collaboration that have had a major impact on the field of cosmic ray physics. As of this writing, 60 full author list papers have been published or accepted, with another 2 submitted and about 7 more in preparation. The Auger Collaboration is also training a cadre of future scientists, with 238 students granted PhDs based on their work on Auger. Another $161 \mathrm{PhD}$ students are in the pipeline. Publications and other technical reports are available online at http://www.auger.org/technical_info/.

\section{Hybrid design}

As indicated above, a key feature of the Pierre Auger Observatory is its hybrid design, in which ultrahigh energy cosmic rays are detected simultaneously by a surface array and by fluorescence telescopes. The two techniques are used to observe air showers in complementary ways, providing important cross-checks and measurement redundancy $[34,35]$.

The surface detector array views a slice of an air shower at ground level, with robust and sensitive water Cherenkov stations which respond to both the electromagnetic and muonic components of the shower. Well-established methods exist for determining arrival directions and for estimating primary energy (see Section 11). The SD operates $24 \mathrm{~h}$ per day and thus provides uniform coverage in right ascension with a huge $3000 \mathrm{~km}^{2}$ collecting area. The instantaneous aperture of the array is easily calculable, especially for energies above $3 \times 10^{18} \mathrm{eV}$, where a shower falling on any part of the array is detected with $100 \%$ efficiency independently of the mass of the primary particle that initiated the shower. The aperture is found simply by counting the number of hexagons of active surface stations at any time, and multiplying by the aperture, $A \Omega$, of a hexagonal cell, $4.59 \mathrm{~km}^{2} \mathrm{sr}$ (for shower zenith angles $<60^{\circ}$ ) [36]. The SD has the important property that the quality of the measurements improves with the shower energy.

The fluorescence detector is used to image the longitudinal development of the shower cascade in the atmosphere. The fluorescence light is emitted isotropically in the ultraviolet part of the spectrum and is produced predominantly by the electromagnetic component of the shower. Observation periods are limited to dark nights of good weather, representing a duty cycle which has increased from $12 \%$ during early years [37] up to $\sim 15 \%$ at the present time (see Section 12). This disadvantage is balanced by the considerable gain of being able to view the development of the shower profile. Firstly, since fluorescence light production is proportional to the collisional energy deposit in the atmosphere, the technique provides a near-calorimetric method for determining the primary cosmic ray energy. Secondly, the depth at which a shower reaches maximum size, $X_{\max }$, is observable: this is the most direct of all accessible mass composition indicators.

The aim is to use the FD and the SD to measure the same properties of primary cosmic rays (energy, mass composition, and direction) but to do so using different techniques with very different systematic uncertainties. Thus, part of the function of the fluorescence detector is to enable cross-checks to be made and to train the surface array, providing confidence in the SD measurements made during the majority of the time when no fluorescence detector is operating. However, the fluorescence detector is much more than a calibration tool. The data set collected during hybrid observations is of high quality, being especially useful for those studies that require more precise shower directions than are 
available from the surface array alone and for studies where longitudinal profile measurements are vital.

An example of the synergy between the two techniques is illustrated by measurements of the cosmic ray energy spectrum for showers arriving with zenith angles less than $60^{\circ}$ [38]. The exposure achievable with the surface array is much larger than is possible with hybrid measurements so that in principle a greater number of events can be used in the determination of the spectrum. However, with the surface array alone, there is a serious problem in that the relationship between the primary energy and the SD observable chosen to mirror it, namely the signal measured in the water Cherenkov detectors at $1000 \mathrm{~m}$ from the shower axis, $S(1000)$, can only be found using cascade simulations. This method is inherently unreliable as the necessary hadronic physics is unknown at the energies of interest and it is therefore not even practical to assign a reliable systematic uncertainty. Using the hybrid system, it has proved possible to develop an alternative method for estimating the primary energy that is essentially free from simulations.

The first step is to quantify the dependence of $S(1000)$ with zenith angle. This is done using the "constant intensity" method [39], where the attenuation of the typical air shower with increasing atmospheric depth is mapped out using SD data alone. The conversion to primary energy is then achieved using a subset of SD events also observed with the FD. The only simulation input to the determination of primary energy with the FD is in estimating the small fraction $(\sim 10 \%)$ that goes into neutrinos and high energy muons that continue into the ground. Atmospheric variability (mostly changing aerosol properties) complicates the analysis, due to essential corrections for atmospheric attenuation of the fluorescence light and because of the allowance that must be made for scattered Cherenkov light in the FD signal. The event reconstructions utilize extensive atmospheric monitoring that is performed at the Observatory site whenever the FD operates. The final step in measuring the energy spectrum is a precise determination of the exposure $\left(\mathrm{km}^{2} \mathrm{sr} \mathrm{yr}\right)$ for the observations. As already mentioned, the instantaneous aperture of the SD array is straightforward to calculate, even during the period of construction when it was continually growing. This example of an analysis procedure illustrates the complementary strengths of the SD and FD, and how a robust result can be achieved by drawing on them.

A key to the success of the hybrid technique is that it allows a precise determination of the position of a shower axis in space with an accuracy better than could be achieved independently with either the surface array detectors or a single fluorescence telescope [35]. The first step in geometrical reconstruction makes use of the known orientations of the pixels of the fluorescence detector and of the light intensities registered at the pixels. This enables the showerdetector plane (SDP), the plane in space that contains the shower axis and the FD site, to be determined. Timing information is then used to find the orientation of the shower axis within the SDP (see Section 10). With a FD alone, the accuracy of determining the shower geometry may be poor if the angular length of the observed shower track is short, say less than $15^{\circ}$. In this case, the apparent angular speed of the shower image in the telescope is approximately constant, leading to a degeneracy in the geometry solution. This degeneracy is broken if the angular track length is long enough for a change in the angular speed to be detected, or more robustly, with a measurement of the arrival time of the shower at any point on the ground: thus a timing measurement at a single station of the SD suffices. Using this hybrid reconstruction method, a directional resolution of $0.5^{\circ}$ is routinely achieved [40]. Since only the timing information from a single SD station is needed, the hybrid geometry constitutes an independent and sensitive cross-check on the directional and core location assignments made with the SD. The precise geometry of a hybrid event is also the first step towards a high quality measurement of the longitudinal profile of a shower which, in turn, yields the energy of the primary particle and the depth of maximum $X_{\text {max }}$.

Many experimental challenges exist in fully realizing the promise of the hybrid technique to provide high quality measurements of shower observables. The Collaboration employs a series of crosschecks and measurement redundancies to understand the systematic uncertainties in each measurement. These cross-checks include comparisons between SD and FD measured parameters. There are also important redundancies in various calibration measurements and redundancies in measurements of those atmospheric properties that are critical for accurate FD event reconstruction. Of particular importance is the aerosol content of the atmosphere. The concentration of aerosols is variable over timescales of hours and it can vary over the area of the Observatory. The concentration affects the transmission of fluorescence light from the shower to the FD telescopes, and the scattering of Cherenkov light into the fields of view of the telescopes. While the choice of the number and location of fluorescence sites around the surface array was driven by the desire to minimize the effects of atmospheric uncertainties on reconstruction, a great deal of effort is still required in atmospheric monitoring (see Section 9 for details). The aerosol concentration and distribution are monitored on timescales of $15 \mathrm{~min}$ using the two laser facilities (see Fig. 1) within the detection volume and by lidar systems at each FD site. In addition the directional properties of aerosol scattering are measured at two sites, and the wavelength dependence of the scattering is obtained (Section 9).

While the threshold energy for a fully efficient trigger for showers with zenith angles smaller than $60^{\circ}$ is $3 \times 10^{18} \mathrm{eV}$, hybrid observations require only one SD station to have triggered and thus the threshold for hybrid events is significantly lower. For example, a hybrid measurement of the "elongation rate", the energy dependence of $X_{\max }$, has been published for energies above $10^{18} \mathrm{eV}$ [21], with work underway to push the threshold energy lower with the HEAT fluorescence telescopes (Section 13.1). Hybrid showers are collected with full efficiency over the entire SD array at energies above $10^{19} \mathrm{eV}$. Around 6000 quality hybrid events are recorded per year above $10^{18} \mathrm{eV}$, with 300 per year above $10^{19} \mathrm{eV}$. Quality cuts normally applied to these events include the requirement that the depth of shower maximum is in the field of view of one of the FD telescopes.

One final experimental cross-check is worth mentioning. Though the FD was not designed as a "stereo" instrument, a significant number of showers at the higher energies are observed by more than one FD site. At $10^{19} \mathrm{eV}$ over $60 \%$ of FD showers are viewed in stereo, increasing to $90 \%$ at $3 \times 10^{19} \mathrm{eV}$. Stereo observations provide two or more independent hybrid reconstructions of the shower geometry, and of profile parameters such as energy and $X_{\max }$. This allows cross-checks of atmospheric corrections, and of simulations of the detector resolution. This has confirmed the statistical resolutions for a single site to be $\sim 10 \%$ and $20 \mathrm{~g} / \mathrm{cm}^{2}$ for energy and $X_{\max }$ respectively, at around $10^{19} \mathrm{eV}$ [41].

\section{The surface detector}

\subsection{Overview}

A surface detector station consists of a $3.6 \mathrm{~m}$ diameter water tank containing a sealed liner with a reflective inner surface. The liner contains 12,000 liters of ultra-pure water. Three 9-in. diameter Photonis XP1805/D1 photomultiplier tubes (PMTs) are symmetrically distributed on the surface of the liner at a distance of $1.20 \mathrm{~m}$ from the tank center axis and look downward through windows of clear polyethylene into the water. They record Cherenkov light produced by the passage of relativistic charged 


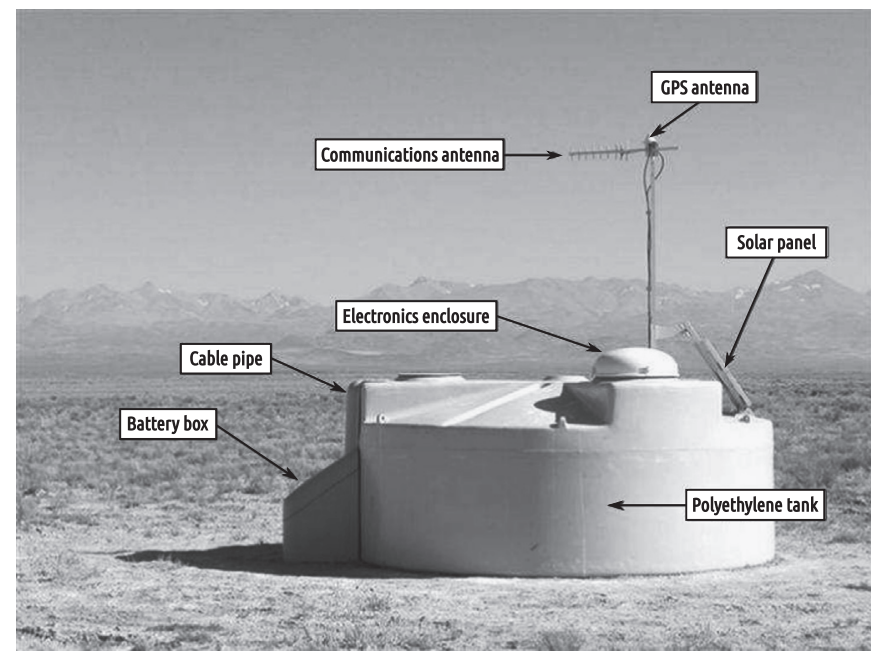

Fig. 3. A schematic view of a surface detector station in the field, showing its main components.

particles through the water. The tank height of $1.2 \mathrm{~m}$ makes it also sensitive to high energy photons, which convert to electronpositron pairs in the water volume.

Each surface detector station is self-contained. A solar power system provides an average of $10 \mathrm{~W}$ for the PMTs and electronics package consisting of a processor, GPS receiver, radio transceiver and power controller. The components of a surface detector station are shown in Fig. 3. Ref. [42] describes the surface detector in detail.

Figure 1 shows the layout of the surface array and the FD buildings at its periphery.

\subsection{The SD station}

The tanks are made of polyethylene using the rotational molding, or rotomolding, process. This process, in simplified form, consists of putting a set amount of polyethylene resin inside a mold, then rotating the mold and heating it until the resin has melted and uniformly coated the interior walls of the mold. The result is a low cost, tough, and uniform tank with robustness against the environmental elements. The carefully selected, custom compounded polyethylene resins contained additives to enhance ultraviolet protection. The interior two-thirds of the wall thickness was compounded with $1 \%$ carbon black to guarantee light-tightness. The outer one-third was colored beige to blend with the landscape. The tanks have an average wall thickness of $1.3 \mathrm{~cm}$ and a nominal weight of $530 \mathrm{~kg}$. The tanks do not exceed $1.6 \mathrm{~m}$ in height so that they can be shipped over the roads within transportation regulations.

Three hatches, located above the PMTs, provide access to the interior of the tank for water filling. They also provide access for installation and servicing of the interior parts. The hatches are covered with light- and water-tight polyethylene hatchcovers. For reasons of cost, durability, and ease of installation, the gaskets sealing the hatchcovers to the tanks may not be perfectly leaktight, so the hatches are elevated to prevent accumulated water from collecting at the gasket. One hatchcover is larger than the other two and accommodates the electronics on its top surface. The electronics is protected by an aluminium dome that keeps out rain and dust. The tanks also possess molded-in lugs, six for lifting and four additional lugs to support the solar panel and antenna mast assembly.

Electrical power is provided by two $55 \mathrm{Wp}$ (watt-peak) solar panels which feed two $12 \mathrm{~V}, 105 \mathrm{Ah}$, lead-acid, low maintenance batteries wired in series to produce a $24 \mathrm{~V}$ system. Power is

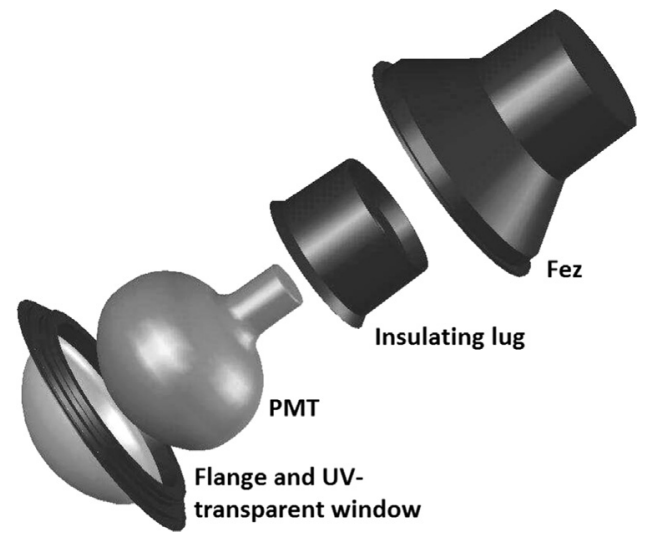

Fig. 4. Mechanical housing for the SD PMT. Top to bottom: outer plastic housing (fez), insulating lug, PMT, flange, and UV-transparent window.

expected to be available over $99 \%$ of the time. Batteries are charged through a commercial charge controller, which is epoxy encapsulated and has robust surge protection. The electronics assembly at each SD station possesses a Tank Power Control Board (TPCB) which monitors the power system operation. The TPCB also has a control function which allows the remote operator to set into hibernation any (or all) of the SD stations if the charge of the batteries falls below a critical level. There is enough reserve in the solar power system that this feature has not yet been employed.

The batteries are accommodated in a rotationally molded polyethylene battery box. Since battery lifetime is reduced with increased temperature, the battery box is protected from direct sunlight by installing it on the shaded side of the tank. It is also insulated with polystyrene foam plates to minimize high temperature excursions during the day. The box is anchored by a plate which extends under the tank. Power cables run through the tank interior top from feedthroughs in the large hatch to the far side of the tank, where they exit the tank to run to the battery box. The cables are protected from the point where they exit the tank to the entry of the battery box by a polyethylene pipe.

The solar panels are mounted on aluminium brackets, which also support a mast. Antennas for radio communication and GPS reception are mounted at the top of this mast. The mast-andbracket system is designed to withstand $160 \mathrm{~km} / \mathrm{h}$ winds.

The tank liners are right circular cylinders made of a flexible plastic material conforming approximately to the inside surface of the tanks up to a height of $1.2 \mathrm{~m}$. They enclose the water volume, provide a light-tight environment and diffusively reflect the Cherenkov light produced in the water volume. The liners are produced from a laminate composed of an opaque three-layer coextruded low-density polyethylene (LDPE) film bonded to a 5.6 mils $(0.14 \mathrm{~mm})$ thick layer of DuPont ${ }^{\mathrm{TM}}$ Tyvek $^{\circledR} 1025-\mathrm{BL}^{3}$ by a layer of titanium dioxide pigmented LDPE of 1.1 mils $(28 \mu \mathrm{m})$ thickness. The three-layer coextruded film consists of a 4.5 mils $(0.11 \mathrm{~mm})$ thick carbon black loaded LDPE formulated to be opaque to single photons, sandwiched between layers of clear LDPE to prevent any carbon black from migrating into the water volume. Custom designing the laminate materials has resulted in a durable, flexible liner.

The liner has three windows through which the PMTs look into the water volume from above. These windows are made of UVtransparent linear low-density polyethylene. Each PMT is optically coupled to a window with optical GE silicone RTV-6196 and shielded above by a light-tight plastic cover, designated as the

${ }^{3}$ E.I. du Pontde Nemours and Co., Wilmington, Delaware, USA. www.dupont. com. 
"fez". Figure 4 shows the PMT enclosure. The fez has four ports, including a light-tight air vent for pressure relief. The other ports are for cable feedthroughs. Two foam insulation rings that fit inside the fez serve to prevent ice buildup near the PMT.

Once deployed in their correct positions in the field, the tanks are filled with ultra-pure water produced at a water plant owned and operated by the Auger Collaboration. Water quality (resistivity) exceeds $15 \mathrm{M} \Omega \mathrm{cm}$ at the output of the water plant, and the water is transported in clean specialized transport tanks. Tests have indicated that ultra-pure water does not support bacterial growth which could lead to reduced water clarity. Because of its high purity, the water is expected to maintain its clarity without significant degradation for the lifetime of the Observatory, estimated at 20 years. Occasional testing of the water in a sampling of detectors has shown no significant bacterial growth.

\subsection{The SD electronics}

To collect the Cherenkov light produced in the water volume of the detectors by the air showers, three PMTs view the water volume from above. The PMTs have a 9 in. diameter photocathode and eight dynodes, with the chemical composition of the dynode surfaces optimized by the manufacturer to maximize linearity. Due to their proximity to water they are operated with a positive anode voltage, the photocathode being grounded. The high voltage is provided locally from a module integrated in the PMT base, and is proportional to a DC control voltage provided by the slow control system. The PMTs are operated at a nominal gain of $2 \times 10^{5}$, and are specified for operation at gains up to $10^{6}$. The PMTs are required to be linear within $5 \%$ up to $50 \mathrm{~mA}$ anode current. To minimize the effect of the geomagnetic field on the PMTs, their orientation is aligned with respect to the azimuth of the Earth's magnetic field at deployment. The base, including the high voltage supply, is attached to the tube by soldering to flying leads and is potted in GE silicone RTV-6136 to protect it from the high humidity present in the tank.

Each PMT has two outputs. An AC coupled anode signal is provided. In addition, the signal at the last dynode is amplified and inverted by the PMT base electronics to provide a signal with 32 times the charge gain of the anode. No shaping of the signal is performed on the PMT base.

Six identical channels of electronics are provided to digitize the anode and amplified dynode signals from each of the PMTs. Each channel consists of a 5-pole Bessel filter with a $-3 \mathrm{~dB}$ cutoff at $20 \mathrm{MHz}$ and a voltage gain of -0.5 . This filter is implemented using a pair of Analog Devices AD8012 current feedback op-amps. The filtered analog signals are fed to Analog Devices AD9203 10 bit $40 \mathrm{MHz}$ semi-flash ADCs. The ADC negative inputs are biased to $-50 \mathrm{mV}$ to bring the input pedestal on scale and allow for amplifier section offsets. The choice of filter cutoff results in 5\% aliasing noise while preserving the time structure of the signals. The use of two 10 bit ADCs with a gain difference of 32 extends the dynamic range of the system to 15 bits with a $3 \%$ precision at the end of the overlap region. The maximum signal recorded before saturation corresponds to about 650 times the peak current from a vertical muon traversing the tank, which corresponds to the signal from a $100 \mathrm{EeV}$ cosmic ray at about $500 \mathrm{~m}$ from the shower core.

An LED flasher is mounted in a test port of the water tank liner. The LED flasher incorporates two LEDs which can be pulsed independently or simultaneously and with variable amplitude. This allows testing of the linearity of the photomultipliers to be conducted remotely.

Each SD station contains a GPS receiver with its corresponding antenna mounted at the top of the communications mast for event timing and communication synchronization. The receiver is a Motorola (OEM) Oncore UT + . This receiver outputs a timed one- pulse-per-second (1 PPS). The GPS 1 PPS signal is offset from the true GPS second by up to $50 \mathrm{~ns}$, and a correction for this offset is provided periodically by the receiver. Event timing is determined using a custom ASIC which references the timing of shower triggers to the GPS 1 PPS clock. The ASIC implements a 27 bit clock operating at $100 \mathrm{MHz}$. This clock is latched on the GPS 1 PPS signal at the time of each shower trigger. A counter operating at the $40 \mathrm{MHz}$ ADC clock is also latched on the GPS 1 PPS clock. These data, together with the timing corrections provided by the GPS receiver, are used to calibrate the frequencies of the $40 \mathrm{MHz}$ and $100 \mathrm{MHz}$ clocks and to synchronize the ADC data to GPS time within 10 ns RMS.

The digital data from the ADCs are clocked into a programmable logic device (PLD). In the first half of the deployment, we employed two ALTERA ACEX PLDs (model EP1K100QI208-2) with $16 \mathrm{~K} \times 36$ bits additional external static RAM. In later stations, an Altera Cyclone FGPA replaced the two ACEX devices and external memory. The PLD implements firmware that monitors the ADC outputs for interesting trigger patterns, stores the data in a buffer memory, and informs the station microcontroller when a trigger occurs. There are two local trigger levels (T1 and T2) and a global third level trigger, T3. Details of the local triggers are described in Section 3.5 .

The front end is interfaced to a unified board which implements the station controller, event timing, and slow control functions, together with a serial interface to the communications system. The slow control system consists of DACs and ADCs used to measure temperatures, voltages, and currents relevant to assessment of the operation of the station.

The station controller consists of an IBM PowerPC 403 GCX$80 \mathrm{MHz}$, with a $32 \mathrm{MB}$ DRAM bank to store data and executable code, and a 2 MB Flash EPROM for the bootstrap and storing of the OS9 operating system. The data acquisition system implemented on the station controller transmits the time stamps of the $\sim 20 \mathrm{~T} 2$ events collected each second to CDAS (central data acquisition system; see Section 6). CDAS returns T3 requests to the station within $\sim 8 \mathrm{~s}$ of the event (including communication delay due to retransmission). The station controller then selects the T1 and T2 data corresponding to the $\mathrm{T} 3$ requests and builds it into an event for transmission to CDAS. Calibration data are included in each transmitted event.

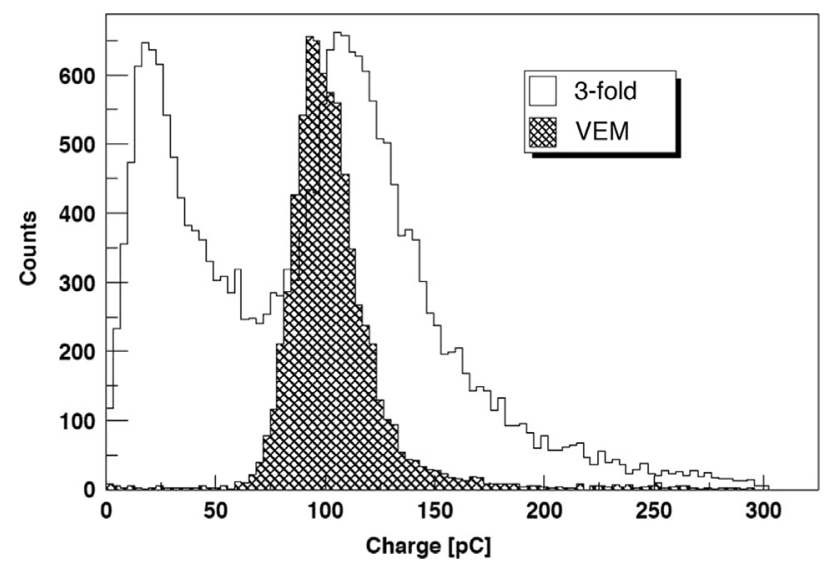

Fig. 5. Charge spectrum obtained when a surface detector is triggered by a 3-fold coincidence among its photomultipliers (open histogram). The hatched histogram shows the spectrum when triggered on central vertically aligned plastic scintillators. The bin containing the peak of the scintillator triggered spectrum is defined as a vertical equivalent muon. The leftmost peak in the open histogram is due to low energy and corner-clipping muons convolved with the 3-fold low threshold coincidence. 


\subsection{Calibration of the surface detector}

The Cherenkov light recorded by a surface detector is measured in units of the signal produced by a muon traversing the tank on a vertical trajectory (Fig. 5). This unit is termed the vertical equivalent muon (VEM). The goal of the surface detector calibration is to measure the value of $1 \mathrm{VEM}$ in hardware units (i.e., in integrated FADC channels). During shower reconstruction, the signal recorded by the tanks is converted into units of VEM, and the total shower energy and arrival direction are fitted using a lateral distribution function and energy conversion based upon hybrid analysis using the FD. The conversion to units of VEM is done both to provide a common reference level between tanks and to calibrate against the detector simulations.

The total bandwidth available from each SD station to the CDAS is $\sim 1200 \mathrm{bit} / \mathrm{s}$, which requires that the calibration be done by the local electronics. Also, the remoteness of the detectors implies that the calibration procedure be robust, allowing for failures of individual PMTs.

We define $Q_{V E M}^{\text {peak }}$ (denoted simply by $Q_{V E M}$ hereafter) as the bin containing the peak in the charge histogram of an individual PMT response, and $I_{\mathrm{VEM}}^{\text {peak }}$ (denoted by $I_{\mathrm{VEM}}$ hereafter) as the bin containing the peak in the pulse height histogram. These quantities are used in the three main steps in the calibration procedure:

1 Set up the end-to-end gains of each of the three PMTs to have $I_{\mathrm{VEM}}$ at 50 channels. The choice of $50 \mathrm{ch} / I_{\mathrm{VEM}}$ results in a mean gain of $\sim 3.4 \times 10^{5}$ for a mean $n_{\mathrm{pe}} / \mathrm{VEM}$ of $\sim 94$ photoelectrons.

2. To compensate for drifts, adjust the electronics level trigger by continually performing a local calibration to determine $I_{\mathrm{VEM}}$ in channels.

3. Determine the value of $Q_{V E M}$ to high accuracy using charge histograms, and use the known conversion from $Q_{V E M}$ to 1.0 VEM to obtain a conversion from the integrated signal of the PMT to VEM units.

The high voltages, and thus the gains of each of the three PMTs, are tuned to match a reference event rate. This tuning implies that the PMTs in the SD stations will not have equivalent gains, even for PMTs in the same tank. If, for example, a particular SD station has more detected photons per vertical muon than the average station, then the PMTs in this station will be operated at a lower gain than average to compensate. Conversely, if a PMT has a worse optical coupling than others in the same tank, resulting in fewer observed photons per vertical muon, the PMT will be run at a higher gain.

In addition to the primary conversion from integrated channels to VEM units, the calibration must also be able to convert the raw FADC traces into integrated channels. The primary parameters needed for this are the baselines of all six FADC inputs, and the gain ratio between the dynode and anode. The dynode/anode, or $D / A$, ratio is determined by averaging large pulses and performing a linear time-shifted fit to obtain both $D / A$ and the phase delay between the dynode and anode. This method determines $D / A$ to $2 \%$.

The calibration parameters are determined every $60 \mathrm{~s}$. The most recently determined parameters are returned to CDAS with each event and stored along with the event data. Each event therefore contains information about the state of each SD station in the minute preceding the trigger, allowing for an accurate calibration of the data. Ref. [43] describes in detail the calibration method of the surface detector.

\subsection{The SD local triggers}

Several independent local trigger functions are implemented in the front-end electronics: the scaler trigger, the calibration trigger, and the main shower trigger.
The scaler trigger records pulses with a very low threshold for auxiliary physics purposes such as space weather. The calibration trigger collects low threshold pulses using a small number of bins (20), which is one bin above $0.1 I_{\mathrm{VEM}}$, thus providing high rate cosmic ray data. Data from the three high gain channels are stored from three samples before the trigger to 20 samples after the trigger. These data are used to build calibration histograms such as the one shown in Fig. 5, and are also used to convert offline the six FADC traces into VEM units. It was lowered during the deployment period for investigating signals from the tails of showers and to measure muon decay in the SD water volume [44].

The main trigger is the shower trigger that results in the recording of 768 samples $(19.2 \mu \mathrm{s})$ of the six FADCs. It has two levels of selection. The first level, called T1, has 2 independent modes. The first one is a simple threshold trigger $(\mathrm{TH})$ requiring the coincidence of all three PMTs being above $1.75 I_{\mathrm{VEM}}$. This trigger is used to select large signals that are not necessarily spread in time. It is particularly effective for the detection of very inclined showers that have penetrated through a large atmospheric depth and are consequently dominantly muonic. The threshold has been adjusted to reduce the rate of atmospheric muon triggers from about $3 \mathrm{kHz}$ to $100 \mathrm{~Hz}$. The second T1 mode is a time-over-threshold trigger (ToT) requiring that at least 13 bins within a $3 \mu$ s window (120 samples) exceed a threshold of $0.2 I_{\mathrm{VEM}}$ in coincidence for two out of the three PMTs. The ToT trigger selects sequences of small signals spread in time, and is thus efficient for the detection of vertical events, and more specifically for stations near the core of lowenergy showers, or stations far from the core of high-energy showers. The rate of the ToT trigger depends on the shape of the muon pulse in the tank and averages to $1.2 \mathrm{~Hz}$ with a rather large spread (about $1 \mathrm{~Hz}$ rms). The second trigger level, called T2, is applied to decrease the global rate of the T1 trigger down to about $23 \mathrm{~Hz}$. While all T1-ToT triggers are promoted to T2-ToT, only T1-TH triggers passing a single threshold of $3.2 I_{\mathrm{VEM}}$ in coincidence for the three PMTs will pass this second level and become T2-TH. All T2s send their timestamp to CDAS for the global trigger (T3) determination. More details on the triggers can be found in Ref. [36] .

In June 2013, the Observatory installed across the entire array two additional SD T1 triggers. These triggers build upon the ToT trigger in two ways, applying more sophisticated analysis to the FADC traces. The time-over-threshold-deconvolved (ToTd) trigger deconvolves the exponential tail of the diffusely reflected Cherenkov light pulses before applying the ToT condition. This has the effect of reducing the influence of muons in the trigger, since the typical signal from a muon, with fast rise time and $\sim 60$ ns decay constant, is compressed into one or two time bins. The multiplicityof-positive-steps trigger (MoPS), on the other hand, counts the number of positive-going signal steps in two of three PMTs within a $3 \mu$ s sliding window. The steps are required to be above a small FADC value $\left(\approx 5 \times\right.$ RMS noise) and below a moderate value $\left(\approx \frac{1}{2}\right.$ vertical muon step). This reduces the influence of muons in the trigger. Both the ToTd and MoPS triggers also require the integrated signal to be above $\approx 0.5 \mathrm{VEM}$. Because these triggers minimize the influence of single muons, they reduce the energy threshold of the array, while keeping random triggers at an acceptable level. Thus they improve the energy reach of the SD, as well as improve the trigger efficiency for photon and neutrino showers.

\subsection{Operation and maintenance}

Currently more than 1660 surface detector stations are operational. Concerning the water Cherenkov detectors themselves, only very few failures have been detected. Only a few liners were observed to leak shortly after installation. In this case, which constitutes the worst failure mode, the tank is 
emptied and brought back to the Assembly Building for replacement of the interior components.

The electronics of the surface detector operates using solar power. A tank power control board incorporates protection circuits, signal conditioning for the monitoring of the solar power system, and a circuit allowing for orderly shutdown and wakeup of the station in the event of an extended cloudy period during winter when there could be inadequate solar power available to operate the station continuously. The solar power system has not yet experienced a dark period long enough to require shutting down the array for battery recharging. The most probable battery lifetime is 4.5-6 years [45], and batteries are changed during regular maintenance trips.

The PMTs and electronic boards are the most critical elements of the SD stations. They are subject to very severe environmental conditions: temperature variations, humidity, salinity and dust. The failure rate of the PMTs is about 20 per year (about $0.5 \%$ ). Some high voltage (HV) module and base problems have been detected as well as some problems due to bad connections. All other failures except those concerning the PMTs (such as a broken photocathode) can be repaired in the field. It is currently estimated that the number of spare PMTs is sufficient for about 10-15 more years of operation. The failure rate of electronic boards is about $1 \%$ per year. Some of the problems are repaired simply by reflashing the software. Most of the electronic problems can also be repaired on site. All the spare parts are stored in Malargüe.

The operation of the array is monitored online and alarms are set on various parameters [46]. The maintenance goal is to have no more than 20 detector stations out of operation at any time. Currently the achieved number is less than 10 detector stations out of operation. It is currently estimated that the long-term maintenance (including the battery change) requires about 3 field trips per week. This maintenance rate is within the original expectations. The maintenance is organized by the Science Operation Coordinator and performed by local technicians. The surface detector does not require a permanent presence of physicists from other Auger institutions on site.

\section{The fluorescence detector}

\subsection{Overview}

The 24 telescopes of the FD overlook the SD array from four sites - Los Leones, Los Morados, Loma Amarilla and Coihueco [47]. Six independent telescopes are located at each FD site in a clean climate controlled building [33], an example of which is seen in Fig. 6. A single telescope has a field of view of $30^{\circ} \times 30^{\circ}$ in azimuth and elevation, with a minimum elevation of $1.5^{\circ}$ above the horizon. The telescopes face towards the interior of the array so that the combination of the six telescopes provides $180^{\circ}$ coverage in azimuth.

\subsection{FD telescopes}

The details of the fluorescence detector telescope are shown in Fig. 7 and an actual view of an installed telescope in Fig. 8. The telescope design is based on Schmidt optics because it reduces the coma aberration of large optical systems. Nitrogen fluorescence light, emitted isotropically by an air shower, enters through a circular diaphragm of $1.1 \mathrm{~m}$ radius covered with a Schott MUG-6 filter glass window. The filter transmission is above $50 \%(80 \%)$ between 310 and $390 \mathrm{~nm}$ (330 and $380 \mathrm{~nm}$ ) in the UV range. The filter reduces the background light flux and thus improves the signal-to-noise ratio of the measured air shower signal. It also serves as a window over the aperture which keeps the space

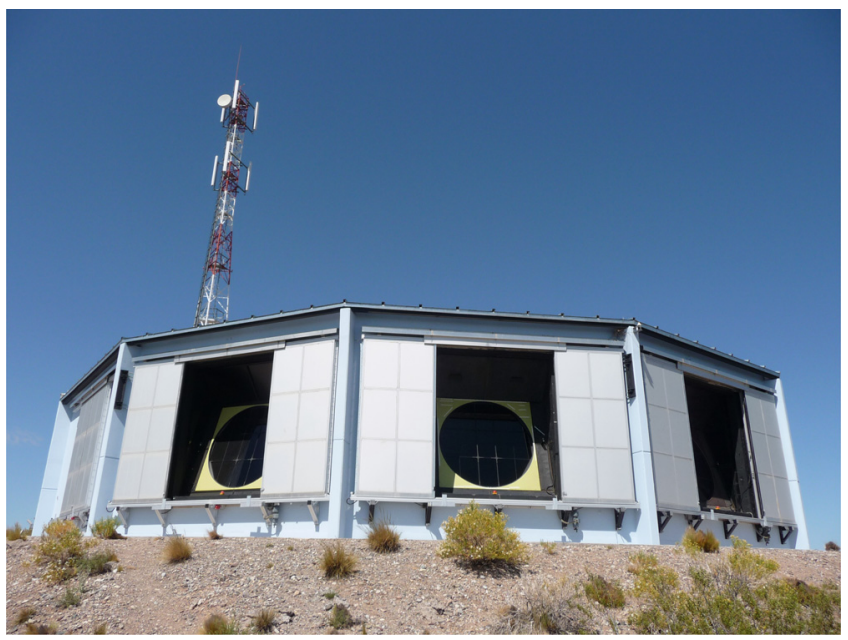

Fig. 6. FD building at Los Leones during the day. Behind the building is a communication tower. This photo was taken during daytime when shutters were opened because of maintenance.

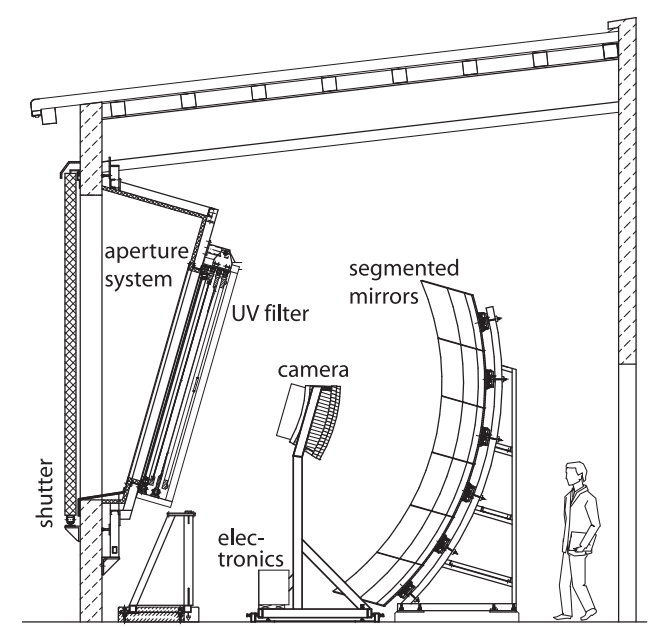

Fig. 7. Schematic view of a fluorescence telescope with a description of its main components.

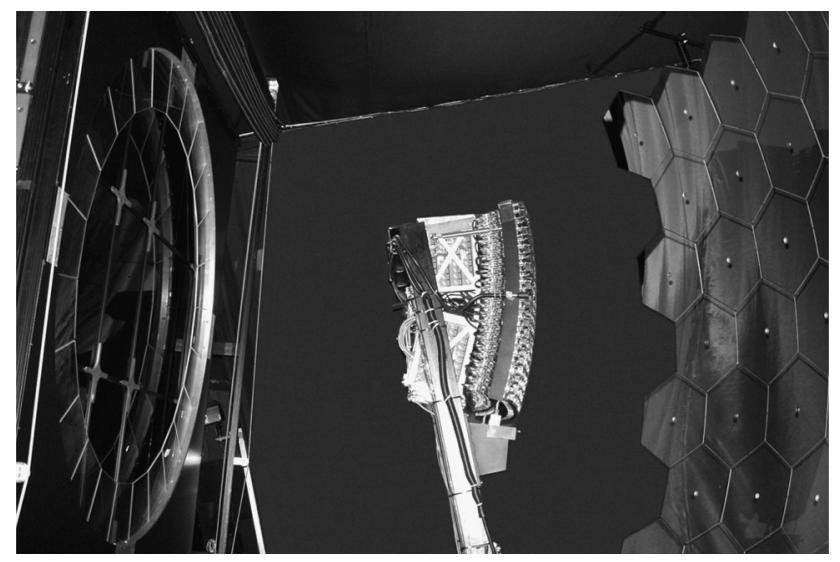

Fig. 8. Photo of a fluorescence telescope at Coihueco.

containing the telescopes and electronics clean and climate controlled. The shutters seen in Fig. 7 are closed during daylight and also close automatically at night when the wind becomes too high 
or rain is detected. In addition, a fail-safe curtain is mounted behind the diaphragm to prevent daylight from illuminating a camera in case of a malfunction of the shutter or a failure of the slow control system.

A simplified annular lens, which corrects spherical aberration and eliminates coma aberration, is mounted in the outer part of the aperture. The segmented corrector ring has inner and outer radii of 850 and $1100 \mathrm{~mm}$, respectively. Six corrector rings were made from Schott BK7 glass and Borofloat was used for the rest. More details about the corrector ring can be found in $[33,48]$.

The light is focused by a spherical mirror of $\sim 3400 \mathrm{~mm}$ radius of curvature onto a spherical focal surface with radius of curvature $\sim 1700 \mathrm{~mm}$. Due to its large area $\left(\sim 13 \mathrm{~m}^{2}\right)$, the primary mirror is segmented to reduce the cost and weight of the optical system. Two alternative segmentation configurations are used: one is a tessellation of 36 rectangular anodized aluminium mirrors of three different sizes; the other is a structure of 60 hexagonal glass mirrors (of four shapes and sizes) with vacuum deposited reflective coatings [33]. The average reflectivity of cleaned mirror segments at a wavelength $\lambda=370 \mathrm{~nm}$ is more than $90 \%$.

The camera body is machined from a single aluminium block of $60 \mathrm{~mm}$ thickness, with an outer radius of curvature of $1701 \mathrm{~mm}$ and an inner curvature radius of $1641 \mathrm{~mm}$. The hexagonal photomultiplier tubes, model XP3062 manufactured by Photonis, are positioned inside $40 \mathrm{~mm}$ diameter holes drilled through the camera block at the locations of the pixel centers. The pixels are arranged in a matrix of 22 rows by 20 columns.

The PMT boundaries are approximate hexagons with a side to side distance of $45.6 \mathrm{~mm}$. The PMTs are separated by simplified Winston cones secured to the camera body which collect the light to the active cathode of the photomultiplier tube. The light collectors serve to prevent photons from landing in the dead spaces between the PMT cathodes. The upper edge of the light collectors lie on the focal surface of $1743 \mathrm{~mm}$ radius. The pixel field of view defined by the upper edges corresponds to an angular size of $1.5^{\circ}$.

All support structures and cables are distributed so as to minimize any obscuration in the light path. The contribution of reflection and scattering inside the optical system of the telescope has been measured in situ and with an airborne remotely controlled platform carrying an isotropic and stabilized UV light source [49]. The measured point spread function of the light distribution in pixels has been implemented in the software used in the air shower reconstruction.

Cleaning and maintenance work has been required during years of detector operation. The cleaning of the UV filter from outside has been performed several times because of deposited dust layers. Currently, the cleaning of all UV filters from outside is done three times per year. The equipment inside the building (i.e., the inner side of the filter, the corrector ring, and the dust curtain) is cleaned less frequently, because it is not exposed to the outside environment. Dry and wet methods have been adopted for cleaning the mirror segments and they both improve the reflectivity of mirrors. For telescopes, where the first cleaning took place six years after their installation, the reflectivity increased by $\leq 1 \%$ in the case of mirror segments in the upper rows and $\sim 5 \%$ for mirror segments in the bottom rows, where the segments are turned slightly upward (see, e.g., Fig. 8). The reflectivity of a few selected mirror segments is measured once or twice each year and it changes less than $1 \%$ per year.

Alignment of individual mirror segments was cross-checked with a laser on site. Moreover, additional methods using data measured by telescopes were used, such as star tracking, Central Laser Facility (CLF) and eXtreme Laser Facility (XLF) shots (Section 4.4.2), or a comparison of FD and SD geometry reconstruction. Only in two cases were a realignment of a telescope and a readjustment of camera position needed.

\subsection{FD electronics}

The FD electronics must provide a large dynamic range and strong background rejection, while accepting any physically plausible air shower. Moreover, the electronics is responsible for antialias filtering, digitizing, and storing signals from the PMTs.

The XP3062 photomultiplier tube is an 8-stage unit with a bialkaline photocathode with quantum efficiency of about $25 \%$ in the wavelength range $350-400 \mathrm{~nm}$. The PMT high voltage is provided by a HV divider chain which forms a single physical unit together with the signal driver circuitry. This head electronics unit is soldered to the flying leads of the PMT [50].

The nominal gain for standard operation of the FD is set to $5 \times 10^{4}$. Stabilization of the HV potential for large pulses, and in the presence of the low but not negligible light intensity of the dark sky background, is realized by employing an active network that uses bipolar transistors in the last three stages of the PMT. The active divider ensures that the gain shift due to the divider chain is less than $1 \%$ for anode currents up to about $10 \mathrm{~mA}$. The normal dark sky background on moonless nights induces an anode current of about $0.8 \mu \mathrm{A}$ on each PMT.

The head electronics for each PMT is connected to a distribution board located just behind the camera body. Each board serves 44 PMTs, providing high and low voltage and receiving the output signals. The signal is then shaped and digitized in the front-end electronics (FE) unit, where threshold and geometry triggers are also generated. Analog boards in the FE unit are designed to handle the large dynamic range required for air fluorescence measurements; this means a range of 15 bits and $100 \mathrm{~ns}$ timing.

As the PMT data are processed, they are passed through a flexible three-stage trigger system implemented in firmware and software. The trigger rate of each pixel in a camera (first level trigger) is kept around $100 \mathrm{~Hz}$ by adjusting the pixel threshold level. The algorithm of the second level trigger searches for track segments at least five pixels in length within a camera. The typical trigger rate per camera fluctuates between 0.1 and $10 \mathrm{~Hz}$. The third level trigger is a software algorithm designed to clean the air shower data stream of noise events that survive the low-level hardware triggers. It is optimized for the fast rejection of triggers caused by lightning, triggers caused by cosmic ray muon impacts on the camera and randomly triggered pixels.

A rugged commercial computer (MPC) is associated with each telescope and serves to readout the event data from the front-end electronics through a FireWire interface. The MPCs at each FD site are connected through a 100 Mbit Ethernet LAN switch to the site's central readout computer, called an "EyePC". This PC provides a connection between the communications network and the MPCs. The MPCs are diskless, thus they boot their Linux system through the network and store their data on the EyePC's hard drive.

The events surviving all trigger levels are sent through the MPC to the EyePC, which builds an event from the coincident data in all telescopes at a given site and generates a hybrid trigger (FD-T3) for the surface array. The event rate is about $0.012 \mathrm{~Hz}$ per site for the 24 baseline telescopes (see Section 12).

\subsection{FD test and calibration}

\subsubsection{Laser test beams}

Throughout each night of FD operation, thousands of collimated UV laser pulses are directed into the atmosphere from two facilities located near the center of the SD (see Fig. 1 and Fig. 29 in Section 9). Light scattered out of the laser pulses generates tracks in the same FD telescopes that also record the tracks generated by air showers. In contrast to high energy air showers, the direction, rate, and energy of the laser pulses can be preprogrammed as desired. Laser pulses can be fired at specific directions relative to 
the ground, for example vertically, or in specific directions relative to the sky, for example aimed at the galactic center, Cen A or other potential sources of cosmic rays. An optical fiber at each laser directs a small amount of light into an adjacent SD station to provide hybrid laser events.

Laser data recorded by the FD telescopes are used to measure FD performance, measure SD-FD time offsets, check FD pointing, and make the hourly measurements of aerosol optical depth vertical profiles for the atmospheric database. In addition, a low power roving system is available for use on a campaign basis.

\subsubsection{CLF and XLF}

Laser test pulses are provided by the CLF [51] and XLF, each of which features a Q-switched frequency tripled YAG laser. The spectral purity of the $355 \mathrm{~nm}$ light pulses delivered to the sky is better than $99.9 \%$. This wavelength falls near the middle of the nitrogen UV fluorescence spectrum, between the two major $\mathrm{N}_{2}$ fluorescence bands of $337 \mathrm{~nm}$ and $357 \mathrm{~nm}$. The maximum energy per pulse is nominally $7 \mathrm{~mJ}$. Pulses of this energy produce tracks in the FD that have an approximate optical equivalence of $100 \mathrm{EeV}$ air showers. The CLF has been in operation since 2003. The XLF was installed in 2008 and includes an automated calibration system that measures the beam energy and polarization. A major upgrade to the CLF in 2013 added a beam calibration system and a backscatter Raman lidar receiver.

\subsubsection{FD detector calibration}

The precise reconstruction of air shower longitudinal profiles requires the conversion of an $\mathrm{ADC}$ count to a light flux for each pixel that receives a portion of the signal from a shower. To this end, the absolute calibration of the detector response is essential. A calibrated large diameter, drum shaped light source provides an absolute, end-to-end calibration for each pixel of the fluorescence telescopes, with independent verification for some pixels by atmospheric Rayleigh scattering from vertical laser pulses.

For these absolute methods, the flux of photons on the telescope aperture is independently measured. The effects of diaphragm area projection, optical filter transmittance, mirror reflectivity, pixel light collection efficiency and area, cathode quantum efficiency, PMT gain, preamp and amplifier gains, and digital conversion are all included in the end-to-end calibration procedure.

The drum light source consists of a pulsed UV LED, emitting in a narrow band around $365 \mathrm{~nm}$, mounted in a cylindrical shell of Teflon ${ }^{\circledR}$, illuminating the interior of the $2.5 \mathrm{~m}$ diameter cylindrical drum, $1.4 \mathrm{~m}$ deep. The sides and back surfaces of the drum are lined with Tyvek, while the front face is made of a thin sheet of Teflon, which transmits light diffusively. The drum is positioned at the entrance of the telescope under calibration, filling the aperture. Emission from the front face is Lambertian (within 3\%), and provides uniform illumination to each pixel over the full acceptance of the telescope. A schematic of the drum calibration source is shown in Fig. 9.

The drum light source intensity is calibrated [52] to a precision of better than $4 \%$ in a dark room, using a NIST calibrated photodiode as a reference. Absolute calibration constants are obtained from the ratio of the known pulsed flux of photons emitted by the drum and the corresponding ADC pulse integrals of the camera pixels.

Periodically, a Rayleigh calibration system [47] is used as an independent check on the drum light source calibration. The $355 \mathrm{~nm}$ roving laser is positioned a few kilometers from the fluorescence telescope to be calibrated. The laser is directed vertically. The laser beam is depolarized and the pulse-to-pulse intensity monitored to a precision of $5 \%$. The scattered light,
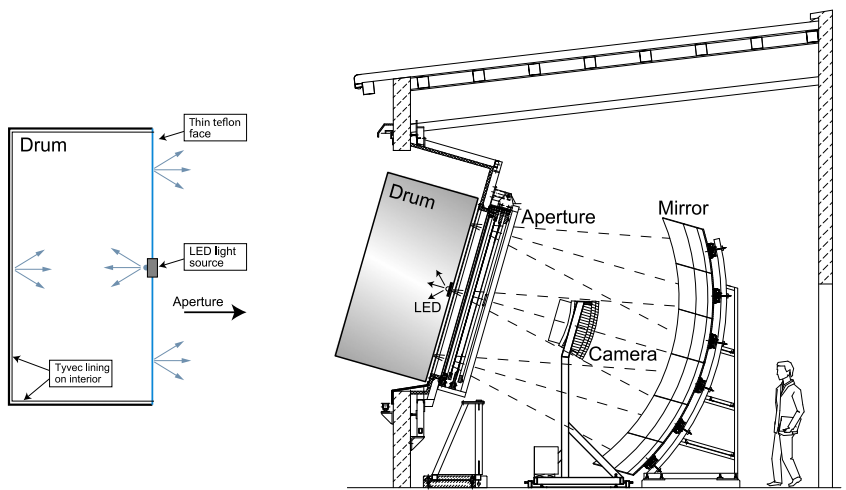

Fig. 9. Drum calibration source for the FD telescopes. Left: Detail of the drum (side view). Right: Sketch of the drum mounted on the telescope window.

mainly from Rayleigh scattering by the molecular atmosphere, is then used to calibrate the fluorescence telescope.

From the end-to-end calibration, the appropriate constants are found to be approximately 4.5 photons/ADC count for each pixel. To derive a flux of photons for observed physics events, the integrated ADC number is multiplied by this constant and divided by the area of the aperture. The flux in photons per $\mathrm{m}^{2}$ perpendicular to the arrival direction is thus obtained.

An optical system for relative calibration [47] is used to monitor the long-term time variations in the calibration of telescopes. In each building, three light sources coupled to optical fibers distribute light signals to three destinations on each telescope. Signals from a pulsed LED light source are brought to a Teflon diffuser at the center of the mirror with the light directed towards the camera. Fibers from a second xenon flash lamp light source end in $1 \mathrm{~mm}$ thick Teflon diffusers at the center of two sides of the camera, with the light directed at the mirror. The signals from the third source, also a xenon flash lamp, are sent to ports on the sides of the entrance aperture where the light is directed toward reflective Tyvek targets mounted on the telescope doors, from which it is reflected back into the telescopes. Drifts of the temporal performance of pixels, mirror and aperture components can be identified by comparing measurements from the three light sources. The sources are also equipped with neutral density filters to permit linearity measurements, or with interference filters to monitor stability at wavelengths in the range of $330-410 \mathrm{~nm}$.

The relative spectral efficiencies, or multi-wavelength calibrations, of FD telescopes were measured using a monochromatorbased drum light source with a xenon flasher. The measurement was done in steps of $5 \mathrm{~nm}$ from $270 \mathrm{~nm}$ to $430 \mathrm{~nm}$. As described in Section 4.2 there are two types of mirrors and two different glass materials used for the corrector rings in the FD telescopes. In total eight telescopes were measured to have a complete coverage of the different components and a redundant measure of each combination. The uncertainty of these measurements is $\sim 3 \%$. An example of measured relative efficiency of an FD telescope is shown in Fig. 10.

\subsection{FD operation}

Beginning in 2005, the FD initially operated with 12 telescopes at two sites, Los Leones and Coihueco (see Fig. 1). In the following years, two additional sites, Los Morados and Loma Amarilla, with six telescopes at each site, were brought into operation. All FD telescopes are operated remotely from the central campus by shift personnel. Their responsibilities include preparation of the FD for a run, making relative calibrations, starting and stopping runs and online checking of the quality of measured data [46]. 
The slow control system (SCS) assures a secure remote operation of the FD system. The SCS works autonomously and continuously monitors detector and weather conditions. Commands from the remote operator are accepted only if they do not violate safety rules that depend on the actual experimental conditions: high voltage, wind speed, rain, light levels inside/outside the buildings, etc. In case of external problems, such as power failures or communication breakdowns, the SCS performs an orderly shutdown, and also a subsequent start up of the fluorescence detector system if the conditions have changed. If parts of the SCS itself fail, the system automatically reverts to a secure mode so that all critical system states (open shutters, high voltage on, etc.) are actively maintained.

The observation of air showers via fluorescence light is possible only at night. Moreover, night sky brightness should be low and thus nights without a significant amount of direct or scattered moonlight are required. We also require that the sun be lower than $18^{\circ}$ below the horizon, the moon remain below the horizon for longer than $3 \mathrm{~h}$, and that the illuminated fraction of the moon be

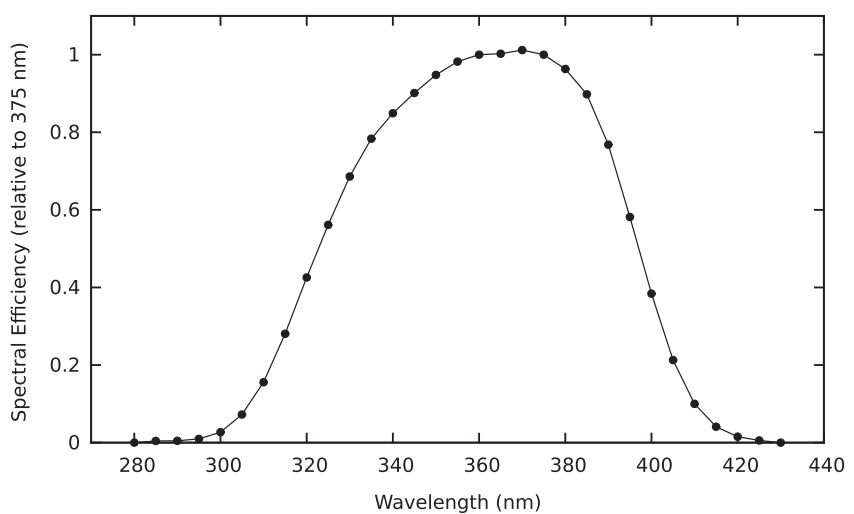

Fig. 10. The relative efficiency between $280 \mathrm{~nm}$ and $430 \mathrm{~nm}$ measured for telescope 3 at Coihueco. The curve is taken relative to the efficiency of the telescope at $375 \mathrm{~nm}$. less than $70 \%$ in the middle of the night. The mean length of the dark observation period is then 17 nights each month.

The on-time of the FD telescopes is currently $\sim 15 \%$. The value varies slightly between telescopes depending on the telescope pointing and various hardware or software factors. The main remaining source of downtime is weather. The telescopes are not operated when the weather conditions become dangerous (high wind speed, rain, snow, etc.) and when the observed sky brightness (caused mainly by scattered moonlight) is too high.

\section{Data communications system}

The detector systems of the Observatory are deployed at widely dispersed positions over a very large area. To send commands and receive data from the four individual FD sites and from 1660 SD stations in the field, a bidirectional radio frequency telecommunication network has been designed and deployed. The reliability of the network is critical to the function of the Observatory, particularly in the context of controlling the experiment, identifying event triggers, and collecting data recorded at each detector for each air shower event.

For Auger, a custom designed system based on a two-layer hierarchy has been implemented. Individual surface detector stations are connected by a custom WLAN which is sectorized and supported by four concentration nodes. The WLAN is serviced by a high capacity microwave backbone network which also supports communications between the four fluorescence detector sites and the main campus data acquisition and control center. Figure 11 shows a conceptual schematic of the overall layout of the data communication system for the Observatory. Table 1 lists the main performance characteristics.

\subsection{The microwave backbone network}

The top layer of the Auger data communications system is a $34 \mathrm{Mbps}$ backbone network made from commercial, point-topoint, dish mounted equipment operating in the $7 \mathrm{GHz}$ band.

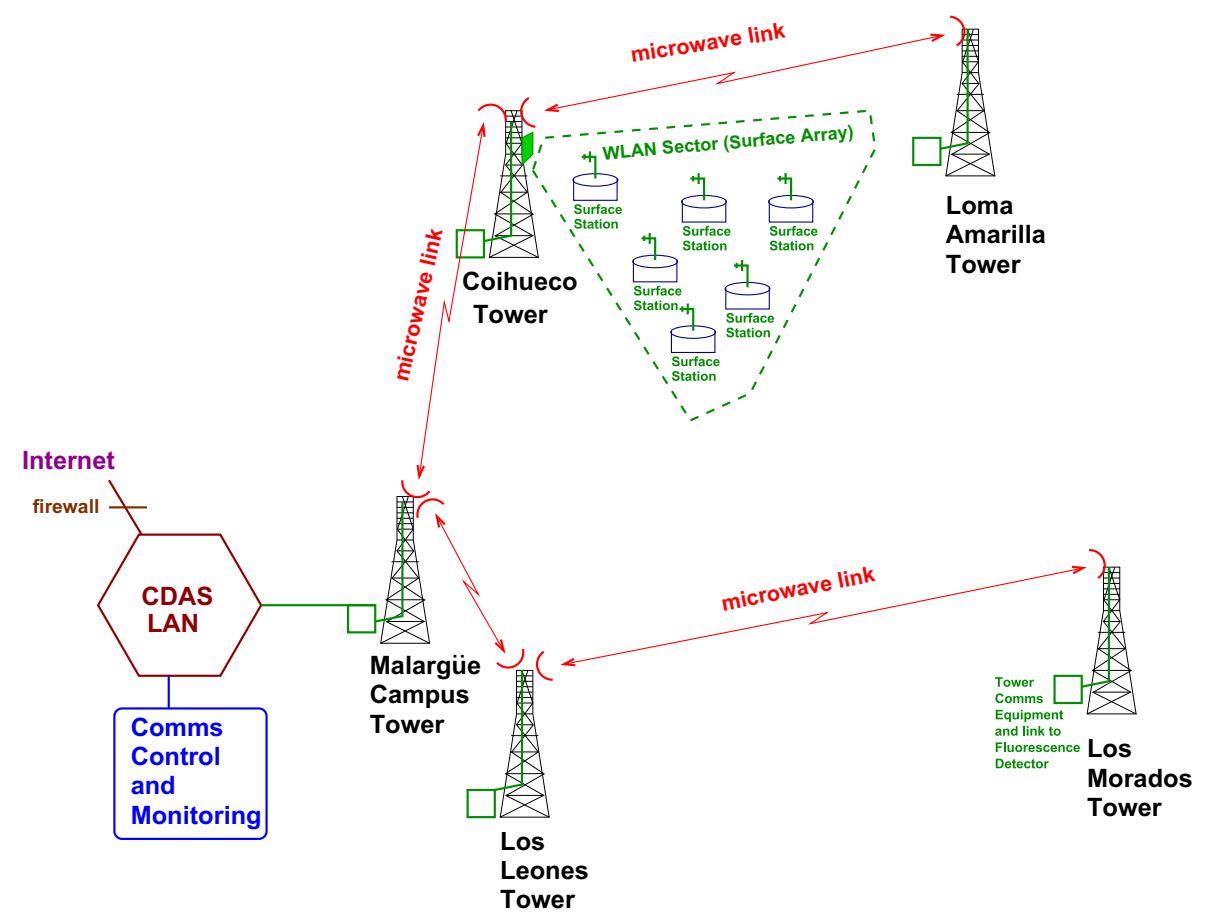

Fig. 11. Conceptual schematic of the overall radio telecommunications system for the Pierre Auger Observatory. 
Table 1

Performance summary for the radio data communications system for the Pierre Auger Observatory.

\begin{tabular}{ll}
\hline Microwave backbone network & \\
\hline Links & 4 \\
Frequency & $7 \mathrm{GHz}$ \\
Data rate & $24 \mathrm{Mbps}$ \\
Wireless LAN & \\
\hline Nodes & 1660 \\
Frequency & $902-928 \mathrm{MHz}$ ISM band \\
Protocol & TDMA, custom \\
Subscriber unit over-air rate & $200 \mathrm{kbps}$ \\
Effective payload rate & 1200 bps uplink \\
Typical daily data packet loss rate & Less than $0.002 \%$ \\
\hline
\end{tabular}

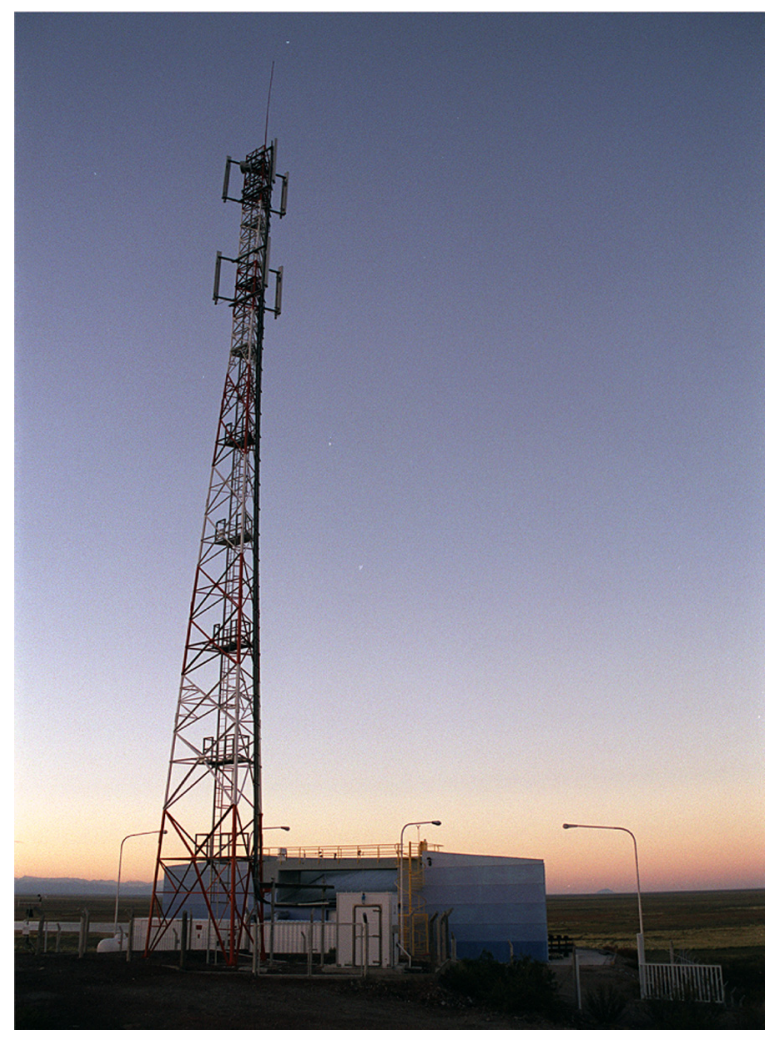

Fig. 12. One of the five communications towers: the one shown is deployed at the Los Leones site; see Fig. 11.

Receivers and transmitters are mounted on five communications towers located at the perimeter of the array as depicted in Fig. 12. The microwave backbone provides high speed network communications to nodes at all four FD sites and with the main campus.

The microwave backbone, depicted schematically in Fig. 13, consists of a set of paired links providing sufficient capacity to stream data to and from each of the FD sites as well as for collecting data from the individual surface stations.

\subsection{Wireless LAN}

The bottom layer of the Auger communication system consists of an extended WLAN comprising custom designed units operating in the 902-928 MHz ISM band. A point-to-point bidirectional communications link is established between each surface detector station and one of four communication concentrator nodes
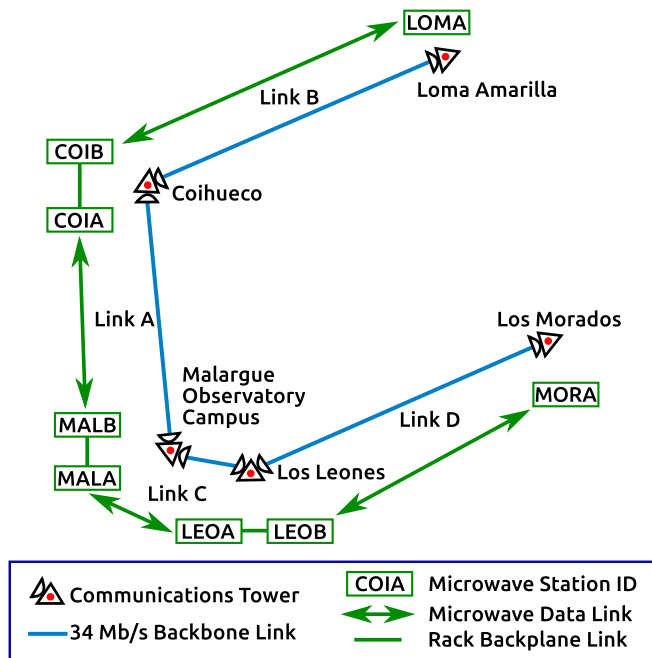

Fig. 13. Configuration of the high capacity microwave backbone network that connects the four FD sites with the main campus control and data acquisition center in Malargüe.

\begin{tabular}{|c|c|c|c|c|c|c|c|}
\hline $\begin{array}{c}\text { Downlink } \\
\text { Network } \\
\text { Control } \\
\text { Slot }\end{array}$ & $\begin{array}{c}\text { Network } \\
\text { Maintenance } \\
\text { Slot }\end{array}$ & $\begin{array}{c}\text { Uplink } \\
\text { Slots }\end{array}$ & $\begin{array}{c}\text { Downlink } \\
\text { Slots }\end{array}$ & $\begin{array}{c}\text { ARQ } \\
\text { Reply } \\
\text { Slots }\end{array}$ & $\begin{array}{c}\text { Downlink } \\
\text { Slots }\end{array}$ & $\begin{array}{c}\text { ARQ } \\
\text { Reply } \\
\text { Slots }\end{array}$ & $\begin{array}{c}\text { CPS } \\
\text { Oscillator } \\
\text { Tuning } \\
\text { Slots }\end{array}$ \\
(1 slot) & $(1$ slot) & (68 slots) & (3 slots) & (3 slots) & (3 slots) & (3 slots) & (3 slots) \\
\hline
\end{tabular}

85 Timeslots $=1$ Frame (duration 1 second)

Fig. 14. A single GPS synchronized $1 \mathrm{~s}$ TDMA frame is broken in time slots as shown.

mounted on the four towers located at each of the fluorescence detector sites. Communication is achieved in a manner similar to a cellular telephone system by dividing the array into 28 sectors, each of which contains up to 68 stations.

Communications operations at each surface station are governed by a custom-built programmable subscriber unit used to mediate the transmission and reception of digital data between the electronics board of a surface detector and the concentrator node. An analogous custom-built unit, called a base station unit, mediates data transfer between each concentrator node and the backbone network connection at each tower.

\subsection{Time division multiple access}

Transmissions to and from the stations are synchronized by GPS timing so that each station is assigned a particular time slot during which it is available to send and receive data. This time division multiple access (TDMA) scheme provides a contention free communication environment within the array. A one-second data frame includes 68 uplink slots for collecting data from the array and 6 downlink slots for sending trigger requests and other commands to the stations. An additional 11 slots are reserved for network management, monitoring, and packet error control. The assignment of individual time slots within the 1-s TDMA frame is shown in Fig. 14. This makes available an effective bandwidth of at least 1200 bps uplink for each surface station and a 2400 bps for broadcast downlink.

\subsection{Error handling}

A central requirement of the Auger WLAN system for collecting data from the surface detector stations is very high reliability. In the wake of a typical trigger, digitized data from PMT traces and other detector information must be relayed promptly to the CDAS 
so that the event can be built and recorded. Data from a single event trigger will typically be broken into several dozen packets transmitted by each station on request, a process that can continue for as long as $2 \mathrm{~min}$. If even a single data packet is missing or corrupt, the entire trace from the station is lost. A custom packetization protocol that includes Cyclic Redundancy Checking to detect transmission errors is used at every level. An advanced retransmit-on-error scheme, commonly called an Automatic Repeat Request (ARQ), is also employed. The ARQ scheme is especially designed to prevent data loss in the case of variable signal fluctuations, external sources of interference or any other episodic environmental influences. If a packet is flagged as missing or corrupt at the monitoring concentrator of the central network, a request to retransmit the packet is automatically initiated and collected via the subsequent data frame reserved TDMA time slots. The ARQ request will be sent once per frame and will be repeated so that at least six attempts are made to retrieve each missing or corrupt packet.

\section{Central data acquisition system}

\subsection{Overview}

The CDAS has been running since March 2001. The system was designed to assemble the triggers from the surface array detectors, to allow control of these detectors and to organize the storage of data. It is constructed using a combination of commercial hardware and custom made, high level, software components. The system is designed to run continuously, with minimum intervention, with the full 1660 detector array, and can manage many more. Data from the FD are recorded separately at the FD locations and transferred daily to the Computer Center at Malargüe, although hybrid coincidences are identified online within the SD data stream.

The primary role for the CDAS is to combine local trigger information from the SD stations in order to identify potential physical events generating an SD higher level trigger (T3). These triggers combined with the T3 from FD sites (FD T3) are used to generate a request for the relevant data from SD stations for these events. The CDAS is then used to combine and store these data to form a shower event. The CDAS also contains configuration and control mechanisms, the means to monitor system performance, and the tools to access and download SD monitoring, calibration, control and configuration data.

Except for triggering information (see Section 6.3), the CDAS and the FD data acquisition systems are completely independent. The merging of FD and SD data is made offline during the daytime following an FD run. Data are synchronized on the central storage hardware after each night of observation. The newly acquired data within the central storage are mirrored at the primary data mirror located at the Lyon HEP Computer Center (France) every $3 \mathrm{~h}$; later these data can be transferred to secondary mirror sites. The data may then be transferred from a convenient mirror site to over 50 participating institutions.

The communication between applications within the CDAS is controlled using a central message routine manager called the "Information Kernel". This manager allows formatted messages to be broadcast by producer applications (applications that need to advertise their status), and for consumer applications (applications that need to know about the status of others) to receive them on demand. All data, with one exception, are exchanged between the CDAS applications in human readable formatted ASCII and go through the "Information Kernel" manager. The exception is the large binary block of raw data coming from the SD stations. Data exchanged in raw format are calibration blocks and FADC traces (these comprise the event data), data from local triggers as well as the monitoring data.

Since the beginning of 2013, the CDAS runs on a virtual machine using resources within a private Cloud installation. The Cloud is composed of 6 servers, summing up 42 CPUs and 112 GB of RAM. CPUs are $2 \mathrm{GHz}$ or faster. This scheme allows the live migration and automatic failover of virtual machines, to minimize the impact of critical failures. The disk storage system is comprised of redundant disk arrays installed in each server plus some standalone devices, making a total storage space near 8.5 TB, using a shared, replicated and distributed scheme. A Network Time Protocol (NTP) GPS clock is used to synchronize the system times. We have adopted the GNU/Linux Debian latest stable distribution as the operating system, currently v6.0r4. Only a very small fraction of the CPU power is used by the CDAS application processes. Most of the software was developed in $\mathrm{C}$ or $\mathrm{C}++$. The whole system is installed in a Computer Center at the Observatory campus, with controlled temperature and redundant uninterruptible power supply.

\subsection{SD data collection}

The data flow over the radio network, from individual SD stations to the central campus, is controlled by a dedicated application called the "Post Master". The Post Master is the end point of the communication backbone at the Observatory Campus, and is designed to dispatch information extracted from the different data streams of a local station to the other applications of the CDAS. As its name suggests, the Post Master application is used to read the data type contained in a radio frame and to forward it to the proper application within the CDAS so that specific data can be handled. When the data received from individual SD stations are split into several radio frames, they are reassembled and forwarded to clients by the Post Master after all the frames have been received.

The Post Master is used also to route data between the applications of the CDAS and the SD. Commands and configuration parameters can be transmitted, along with event requests, such as the level 3 trigger identified by the "Central Trigger" processor Software downloads over the communications link are also possible, thus enabling upgrades of the local DAQ software at the stations without the need to travel many kilometers to each one.

Data received from each SD station belong to different data streams:

1. Local triggers, T2: the highest priority stream, with a list of time stamps and the type of trigger (threshold or time over threshold), is forwarded to the "Central Trigger" application.

2. Shower data with its calibration data: data in this high priority stream are sent only when a request is received from the CDAS at an SD station. Shower events are split into small pieces and sent together with the $\mathrm{T} 2$ packets so that the available bandwidth is fully used. These data are forwarded to the "Event Builder" application.

3. Control: this is a medium priority stream that describes the state of the detector. It is forwarded to the central "Information Kernel" of the CDAS.

4. Calibration and monitoring information: this is a low priority data stream. It is forwarded to the "Monitoring Recorder" application.

\subsection{The event triggering system}

The triggering system of the Observatory fulfills two conditions. First, it detects showers with high efficiency across the SD array, that is more than $99 \%$ for vertical showers with energy 
above $3 \times 10^{18} \mathrm{eV}$. Second, it allows and identifies cross-triggers (hybrid events) between the FD and SD systems. Triggers from the FD use separate algorithms but are forwarded to the SD system to construct the hybrid data set (see below).

The local DAQ system of each SD station is designed to generate low level triggers (T2) as described in Section 3.5. The time stamps of these triggers are sent every second to the CDAS. The T2 requirements are such that the average rate is always around $20-25 \mathrm{~Hz}$ so that at least $50 \%$ of the bandwidth is free for data transmission. This limitation does not reduce the global trigger efficiency (see below). At the CDAS, the T2s received from all stations are stored in a data block stamped according to the second to which they correspond. This information also allows us to acquire the status of all stations of the array at each second. Once a block has existed for a time greater than the maximum transit time across the radio network ( $5 \mathrm{~s})$, it is transmitted to the "Central Trigger" processor and discarded.

The Central Trigger, or third level trigger T3, initiates the central data acquisition from the array in the three following conditions:

1. A main trigger condition, corresponding to shower candidates, is based on both the time and the spatial clustering of the local triggers received from stations, and is described in detail below.

2. A random trigger is generated every $N$ minutes by selecting one of the T2s in an arbitrary manner, and promoting it to a T3. Currently, $N=30$ but values of 3 and 15 have also been used. The purpose of this trigger is to randomly monitor the FADC traces that satisfy the local trigger conditions and thus to verify the efficiency of the global trigger processor.

3. A 2-fold coincidence within $1 \mu$ s of one of the two doublet stations, two couples of neighboring stations (10 m distant). These occur at $0.8 \mathrm{~Hz}$ and are scaled to $0.0017 \mathrm{~Hz}$ for transmission.

To apply the main trigger condition, the system defines concentric hexagons centered on each station. The "Central Trigger" processor is used to identify groups of stations that are clustered in time and space as SD events. First, time clusters are sought by centering a window of $\pm 25 \mu$ s on each T2. Clusters, with multiplicity of three or more, are then examined for spatial coincidences.

The main trigger condition is satisfied in two modes depending on the local shower trigger conditions. A block diagram illustrating the logic chain and approximate rates of these trigger modes is shown in Fig. 15. The first mode requires the coincidence of at least three detectors that have passed the T2-ToT trigger condition (described in Section 3.5) and that meet the requirement of a minimum of compactness, namely, one of the fired detectors must have one of the other fired detectors in the first hexagon of neighbors while another one is no further than the second hexagon. The second mode is more permissive. It requires a 4-fold coincidence of any type of T2 (T2-ToT or T2-TH) with moderate compactness. Namely, among the four fired detectors, one station may be as far away as the fourth hexagon, if a station is within the first hexagon and another station is no further than the second hexagon. Figure 16 illustrates the geometric requirements of the two trigger modes.

Once the spatial coincidence is verified, final timing criteria are imposed: each T2 must be within $(6+5 n) \mu$ s of the central station, where $n$ represents the hexagon number.

Once a trigger has been identified, a T3 message requesting that all FADC trace information recorded within $30 \mu \mathrm{s}$ of the central T2 is built by the "Central Trigger" processor and forwarded to the "Event Builder" and to the stations by the "Post Master". The trigger message is also stored locally by the "Information Kernel". To select which stations are asked for their traces the system takes each station within six hexagons of each of the stations whose T2 participated in the T3 construction. Additionally, the "Central Trigger" process also stores the number of T2s for each station recorded for monitoring purposes.

With the arrangements described above, the total T3 trigger rate of the Observatory is presently of the order of $0.1 \mathrm{~Hz}$ and about 3 million SD events are recorded yearly.

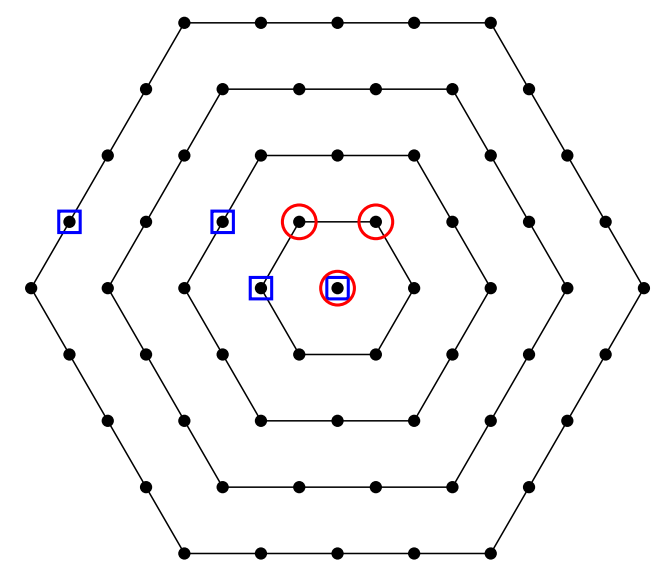

Fig. 16. Four hexagons, containing stations, are illustrated around a central surface station, for a portion of an ideal array. For a 3 -fold coincidence, a T3 is issued if the 3 T2s are ToT, and if one of them is found in the first hexagon of the central station, and the other one no further than the second hexagon. A 4-fold coincidence applies to any kind of T2 and the additional station may be as distant as in the 4th hexagon. Two examples of the topology of triggers are shown: a 4-fold coincidence in which the triggered stations are identified by open blue squares, and a 3-fold coincidence identified by open red circles.



Fig. 15. Schematics of the hierarchy of the trigger system of the Auger surface detector. 
The DAQ system of the fluorescence detector is completely independent of the CDAS. Local triggers are generated at each FD site and those identified as T3 FD event triggers are logged by a local processor if a shower track can be found. T3 FD event triggers are transmitted online (within $1 \mathrm{~s}$ ) from the local FD site to the CDAS system at the central site. The trigger information sent describes the geometry of the shower candidate. This includes the estimated time of arrival of the light front of the shower at the camera as well as the geometry of the SDP (see Section 10.2). From this information, the time of impact of the shower at a ground position in the region of the SD stations is computed and a corresponding SD event T3 is constructed. All FADC traces recorded within $20 \mu \mathrm{s}$ of the computed time are assembled as a normal "SD only" event, but with the addition of the identification of the corresponding T3 FD trigger.

After each night of operation, details of events recorded the FD telescopes for each T3 FD event triggers are transferred to the CDAS. Data from these triggers are then merged with the data collected by the SD DAQ and form the hybrid data set. A hybrid event is therefore an "FD only" event together with a special SD event that contains all the information from the surface stations that were in space and time coincidence with the FD event.

Cross-calibration of the SD and FD clocks is achieved by firing a laser into the sky and, at the same time, injecting a portion of the laser signal into one of the SD stations via an optical fiber. The time of laser pulse emission and the local time stamp recorded in the station are then compared. The former is reconstructed from the laser track recorded in the telescopes and the latter from the local trigger generated by the light going through the tank.

\subsection{Operation and control}

The CDAS has been designed to provide the means to monitor both its own operation as well as the slow control system at SD stations and various environmental parameters. The operation of the CDAS is monitored using a low level application that routinely checks that all software components are running correctly. This "watch dog" system is used to reinitialize and relaunch any application that may have failed. Over the Observatory's life, the CDAS has been operational more than $99.9 \%$ of the time. Most of the downtime is due to system tests, upgrades and debugging with a minimal impact of critical failures.

The manager for the "Information Kernel" system, used to route messages, can also serve as an offline monitoring tool. Its architecture is based upon one central daemon, the message dispatcher, to which all messages are sent automatically and transparently. Any application wishing to distribute its status information, or wishing to know about the state of other applications in the system, connects to the "Information Kernel" and issues a monitoring request defining the class of message it wants to hear.

The "message listener" applications range from monitoring applications to system oriented ones, such as the message logger that is used to make all messages persistent. Applications have been developed that allow these messages to be browsed. Thus, the behavior of the system can be monitored both online and offline. The storage capacity is sufficient to keep the complete history of all messages exchanged in the system (around 3 GB per year of uncompressed ASCII files) for several years.

The "Monitoring Recorder" application of the CDAS is the core of all subsequent monitoring applications that will be described in the next section.

An event display program (part of which can be seen in Fig. 17) has also been developed. This allows the selection, viewing and reconstruction of SD events that are stored on disk. This program, and the input/output and reconstruction libraries that compose it, have also been used extensively for preliminary data analysis.

\section{Monitoring}

\subsection{Overview}

For the optimal scientific output of the Observatory the status of the detector array as well as its measured data have to be monitored. In normal operating conditions, shift personnel or "shifters" monitor the performance of the Auger detector systems. The Auger Monitoring tool has been developed to support the shifter in judging and supervising the status of the detector components, the electronics, communications, and the data acquisition. It is also useful to study "offline" the behavior of the different components of our detectors and to define quality cuts on our data when necessary.

The two hybrid detector systems, SD and FD, are operated differently and therefore the monitoring of their status has different requirements. The stations of the SD array operate continuously in semi-automated mode. Data acquisition must be monitored and failures of any station component (power system, PMT, and communication device) must be detected. The data taking of the FD can only take place under specific environmental conditions and is organized in shifts. The sensitive cameras can only be operated on dark nights without strong winds or rain. This makes the operation a busy task for the shifters who have to judge the operating mode on the basis of the information given by the monitoring system.

The technical description of the implementation is given separately for the services of the central server and the subcomponents. The subcomponents show the different ways the data flow to the central database are organized.

\subsection{Server techniques}

The basis of the monitoring system is a database. We have chosen the widespread and publicly available MySQL database system. It includes all necessary features, e.g., replication and stored functions/procedures. The front end is based on a web server running Apache. The web site uses mainly PHP, CSS and JavaScript. An interface has been developed for generating visualizations. Currently the usage of Gnuplot is implemented via an internal system call. The second option used for generation of visualizations is the JPGraph package which is implemented with direct PHP calls on an object oriented basis with the interface defined in the inherited classes.

Alarms, occurrences of states that require immediate action, are first entered into a specified table of the database. The web front end checks this table for new entries and displays them on the web page. The shifter is trained to observe and acknowledge the alarm; when the problem is solved, he/she can declare the corresponding alarm as resolved.

In addition to the detector performance and data quality monitoring, the functionality of the monitoring server itself has to be guaranteed. Therefore, the central services of the computer have to be monitored to assure that an alarm will definitely be noticed by the shifter.

\subsection{Surface detector}

With large ambient temperature variations, high salinity, dusty air, high humidity inside the tank and remoteness of access, monitoring the performance and reliability of the SD array is a challenge. The temperature ranges from $-15^{\circ} \mathrm{C}$ in winter up to $60{ }^{\circ} \mathrm{C}$ inside the electronics box in summer, with typical daily cycling of about $20^{\circ} \mathrm{C}$. Inside the water tank the relative humidity can reach about $90 \%$. In addition, thunderstorms are rather strong, with lightning that can damage electronic components. 

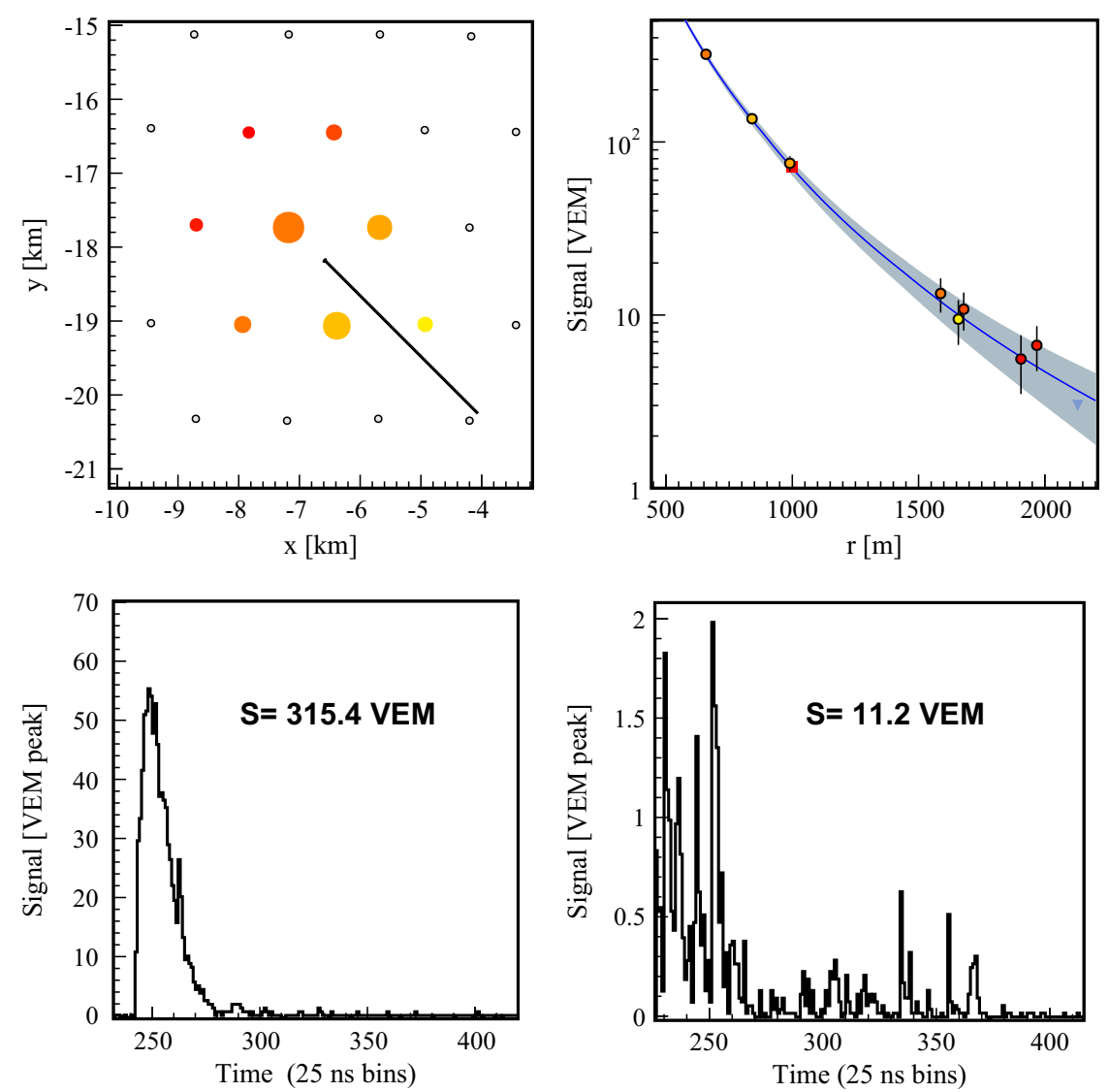

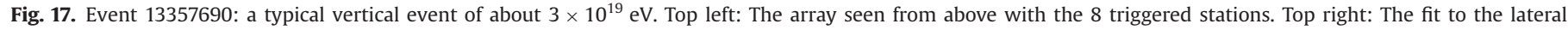

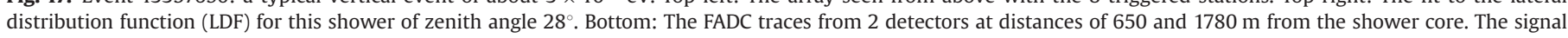
sizes are in units of VEM.

To monitor the whole array accurately, various sensors are installed in every station. Temperature is measured on each PMT base, on the electronics board, and on each battery. PMT voltage and current are also monitored, as well as solar panel voltages, individual battery voltage and charge current. The calibration described in Section 3.4 is performed online every minute. A number of quantities are computed to check the behavior of each water Cherenkov detector: baseline values, single muon peak signal, single muon average charge, dynode/anode ratio and PMT stability. The monitoring and calibration data are sent to the CDAS every $6 \mathrm{~min}$. Dedicated software constantly parses the information sent to the CDAS, independently of the acquisition processes, and exports the data to the MySQL server.

As an example, we show in Fig. 18 the monitoring of the daily average of the voltages of the two batteries of a tank. While the value for Battery 1 is stable above $12 \mathrm{~V}$, it can be seen that since 22 December 2013, the value of Battery 2 has been decaying and is always below $12 \mathrm{~V}$. When the voltage drops below $12 \mathrm{~V}$ for the first time, an alarm is triggered for shifters, so that they can make further checks on the history of the battery to understand the origin of the decay. Once the value becomes lower than $11.5 \mathrm{~V}$, a more severe alarm is triggered to indicate that the battery should be repaired or replaced.

A second example shows how the monitoring system is used to clean the data. Figure 19 displays the evolution of the fraction of PMTs that are rejected by the data analysis due to troubles detected by studying the monitoring data. This number itself is controlled within the monitoring system. The number of lowquality PMTs increased after 2008 because the full array of nearly 5000 PMTs was now deployed, and the Observatory staff needed to carefully balance maintenance priorities. Since 2008 the rate of

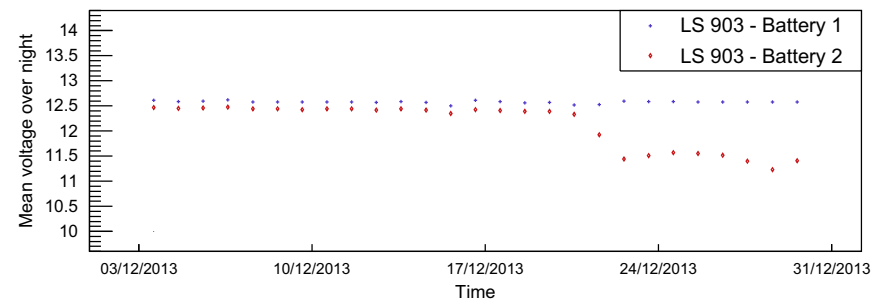

Fig. 18. Evolution with time of the battery average night-time voltage of station 903 in December 2013.

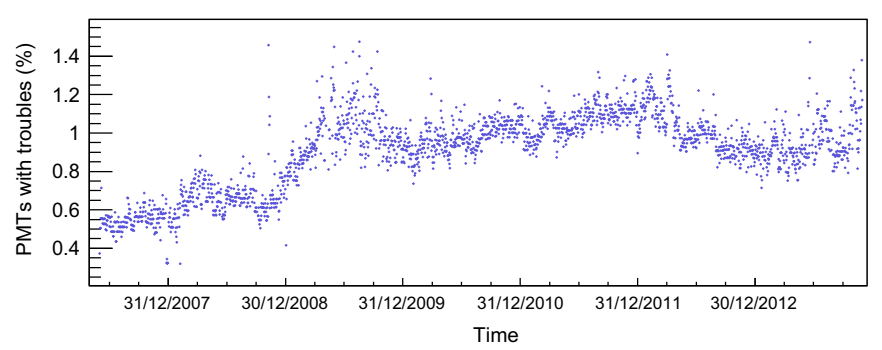

Fig. 19. Percentage of PMTs which do not satisfy the quality criteria among the functioning ones, as a function of time.

low-quality PMTs has been rather stable around 1\%, except for a specific period in 2009 when communication problems did not allow reliable monitoring of the array. We chose to be conservative and disregard doubtful data during this period (see Section 12). 
The monitoring tool also allows a general control of the behavior of the array. Figure 20 shows the muon peak current (IVEM $)$ values for 4802 PMTs. The mean value of the muon peak $\left(I_{\mathrm{VEM}}\right)$ is at channel 45.6 with an RMS of 6.8 showing good uniformity of the detector response. The typical day/night variations are of the order of two ADC channels. This is mainly due to the sensitivity of the PMTs to temperature. The muon calibration is made online every minute, allowing the correction for temperature effects.

The ratio area/peak $(A / P)$, i.e., the ratio between the integrated charge and maximum of the atmospheric muon signals recorded with the calibration trigger, is also a monitored quantity directly available from the local station software. It is related to the water transparency and the reflectivity of Cherenkov light on the inner liner of the SD station. These properties control the absorption length of the light and thus the signal decay constant. Figure 21 shows the decay of the $A / P$ ratio of a typical station in the first seven years following deployment, coupled to a seasonal modulation. After 10 years of deployment, the $A / P$ tends to be stable. This behavior is described in detail in Ref. [45].

The two contributions of the T2 shower triggers (see Section 3.5) are also monitored. While the T2-TH mode has a mean value of $22 \mathrm{~Hz}$ with a low dispersion of less than $2 \%$, the T2-ToT mode contributes only $1 \mathrm{~Hz}$ but with a much larger spread. Indeed, the ToT mode is directly related to the $A / P$, since it is by construction sensitive to the signal shape and thus to the characteristics of the detector. Therefore the T2-ToT rate also decreases with time. It has been observed that, even if the rates of the different stations show a large initial spread across the array, most of them stabilize after a few years to about $1 \mathrm{~Hz}$. Temperature variations also slightly affect the ToT trigger. Fortunately, these variations do not affect the uniformity and the stability of the data, since the event rate above the threshold energy of the experiment has not been affected, as will be shown in Section 12 .

The monitoring tool also includes performance metrics to control the overall performance of the surface detector array. One of the metrics is the number of stations sending a signal

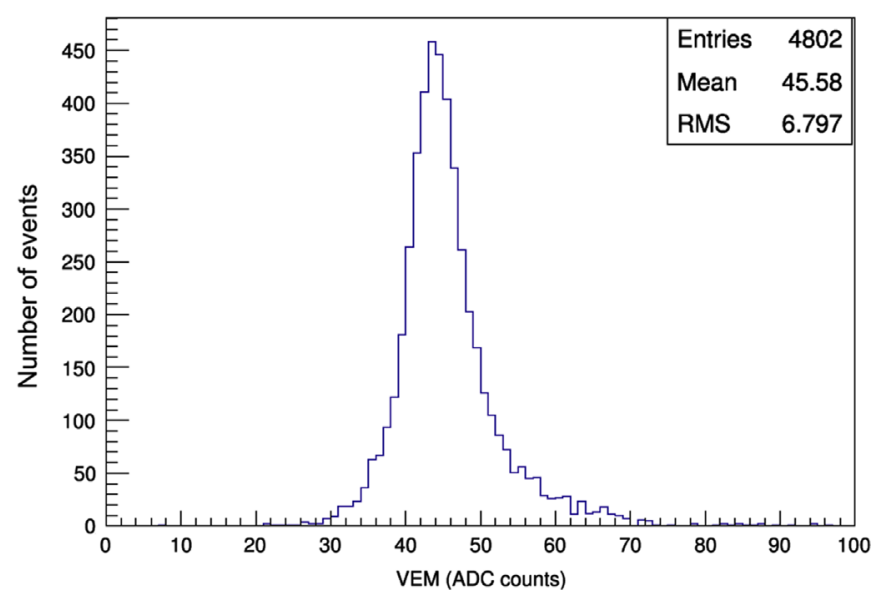

Fig. 20. VEM measured for 4802 PMTs.

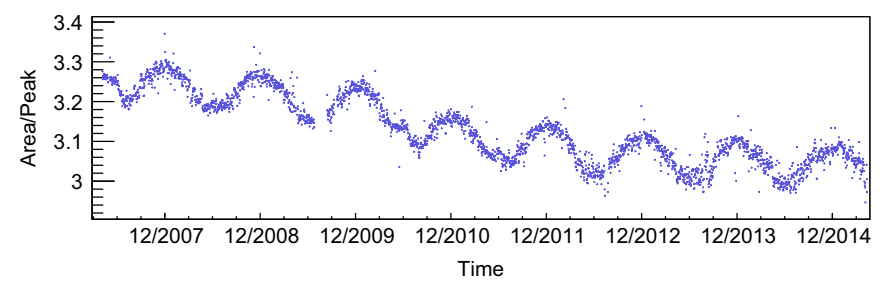

Fig. 21. $A / P$ as a function of time for station 116 . The dots are the average of the $A / P$ value over one day.

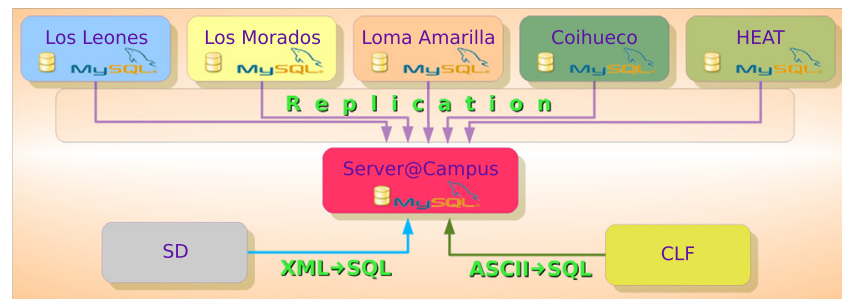

Fig. 22. Organization of the monitoring system databases: The single databases at each FD site are replicated to the database server at the central campus, while other sources like SD fill directly into the database.

divided by the number of deployed tanks as a function of time, indicating the efficiency of data collection with the SD array, which is typically better than $98 \%$.

\subsection{Fluorescence detector}

The data acquisition for the FD telescopes is organized by site to insure against disruption of data collection due to possible communication losses between the CDAS and the remote detectors. The data transport for FD monitoring is organized via a database internal replication mechanism. This mechanism recognizes communication problems and recovers submitted database changes when the connection is reestablished. This guarantees completeness of the dataset on the central server, even if the information is not immediately available online during network failures. Figure 22 shows a schematic layout of the databases.

The information collected for the supervision of the FD operation is organized into five sections. The calibration section contains the information from the different levels of calibration as described in Section 4.4.3, with an example representation given in Fig. 23. The background data section contains the information obtained from each $30 \mathrm{~s}$ readout of the full camera, which is valuable as an unbiased observation of background. The section on DAQ and trigger shows the frequency of fired triggers that indicates the status of the telescopes at an advanced stage. Information from the slow control system such as rain, wind, and outside and inside temperatures is displayed in the fourth section. The lidars [53] monitor the atmosphere close to the telescopes. Their information helps judging the atmospheric conditions at the site, which is vital for the operation of the telescopes.

The data collected in the database can be used to derive higher level quantities such as the on-time of the FD telescopes. This quantity is of major importance since it is a necessary ingredient of flux measurements. The dead time of each telescope is also recorded in the database. Together with the run information and other corrections retrieved from the database, the total on-time for each telescope can be determined individually. The on-time is calculated only for time intervals of $10 \mathrm{~min}$, balancing the statistical precision of the calculated on-time due to statistics with the information frequency. A program to execute the calculation runs on the database server and is used to fill the appropriate tables in the database continuously. The web interface displays the stored quantities. The on-time is available in near real time for the shifter as a diagnostic and figure of merit.

\subsection{Communications}

All aspects of the Auger data communications system control, operations, and performance housekeeping information are coordinated and reported via a central data concentrator node called NetMon, which also serves as the relay for all data transferred between the detectors and the array control center. NetMon enables 


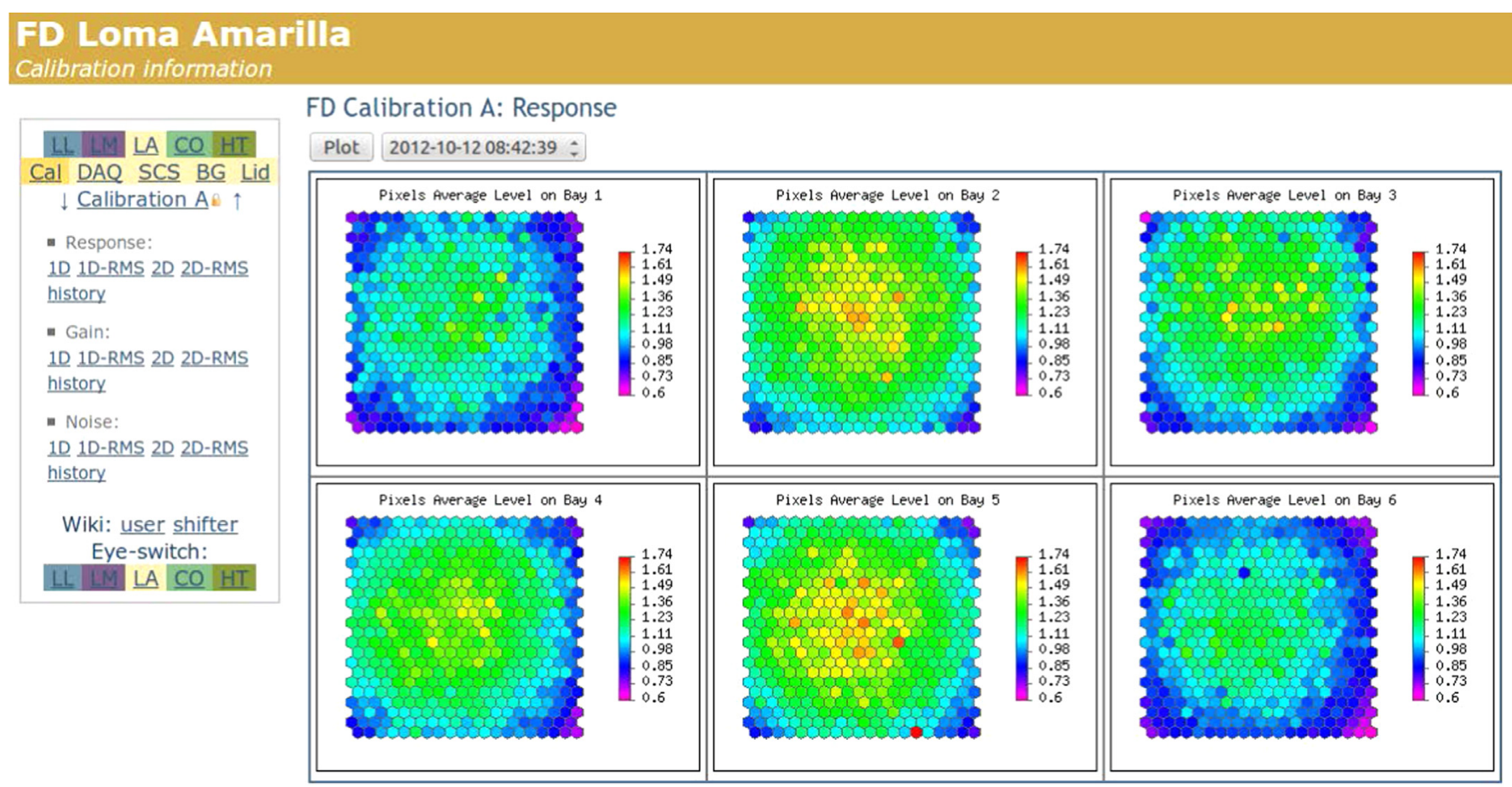

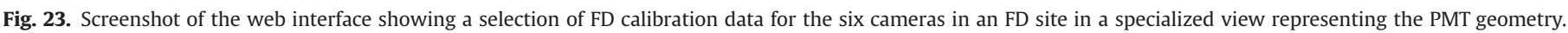

regular monitoring of the system performance, including critical details on the status of data links, the status of sectors and packet error rates. All of this information is integrated into the main monitoring system so that the on site operator can be made aware of any difficulties that may arise in near real time. For example, Fig. 24 shows a monitoring event display of the radio signal strength (in $\mathrm{dB}$ ) of the uplink receiver as reported to the operator for each surface detector station in the array. Long term performance benchmarks for the data communications system are also recorded and monitored. Figure 25 shows the daily number of ARQs over the course of four months of data collected during 2010 and 2011. Here, the ARQ rate serves as a global diagnostic for the overall health of the system indicating the extent to which packets are missing or corrupt. Shown are the rates for single ARQ generating packet errors and also the rates for "ARQ7" errors corresponding to those packets for which at least six retransmission attempts were made before abandoning the packet; the ARQ7 rate thus represents the rate at which data are irretrievably lost. We had for a period in 2009 a large error rate leading to event loss. It has been fixed and since then, the typical loss rate has been approximately 1000-2000 packets out of over 140 million packets per day, corresponding to a data loss rate of less than $0.002 \%$. The data from a typical event involving several stations corresponds to approximately 1000 packets.

Therefore, the overall event loss rate due to communication failures is less than $2 \%$.

\section{Data processing and $\overline{\mathrm{Off}}$ line framework}

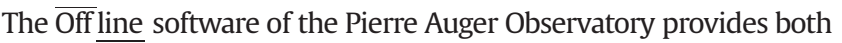
an implementation of simulation and reconstruction algorithms, discussed later, and an infrastructure to support the development of such algorithms leading ultimately to a complete simulation, reconstruction and analysis pipeline. Indeed, when the $\overline{\text { Off line code was }}$ originally devised, the only existing systems were the SD and FD. It has since been extended to handle the radio and AMIGA extensions without requiring dramatic framework changes. The software has been designed to accommodate contributions from a large number of physicists developing $\mathrm{C}++$ applications over the long lifetime of the experiment. The essential features include a "plug-in" mechanism for physics algorithms together with machinery which assists users in retrieving event data and detector conditions from various data sources, as well as a reasonably straightforward way of configuring the abundance of different applications and logging all configuration data. A detailed description of the $\overline{\text { Off }}$ line software design, including some example applications, is available in [54].

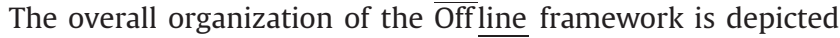

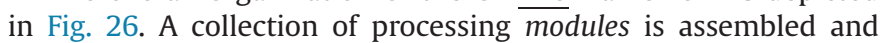
sequenced through instructions contained in an XML file [55] or in a Python [56] script. An event data model allows modules to relay data to one another, accumulates all simulation and reconstruction information, and converts between various formats used to store data on file. Finally, a detector description provides a gateway to data on the detector conditions, including calibration constants and atmospheric properties as a function of time.

\subsection{Physics modules}

Most simulation and reconstruction tasks can be factorized into sequences of processing steps which can simply be pipelined. Physicists prepare processing algorithms in modules, which they register with the Off line framework via a one line macro. This modular design allows collaborators to exchange code, compare algorithms and build up a variety of applications by combining modules in various sequences. Run time control over module sequences is implemented with a Run Controller, which invokes the various processing steps within the modules according to a set of user provided instructions. We devised an XML-based language as one option for specifying sequencing instructions; this approach has proved sufficiently flexible for the majority of our applications, and it is quite simple to use. An example of the structure of a sequence file is shown in Fig. 27. 


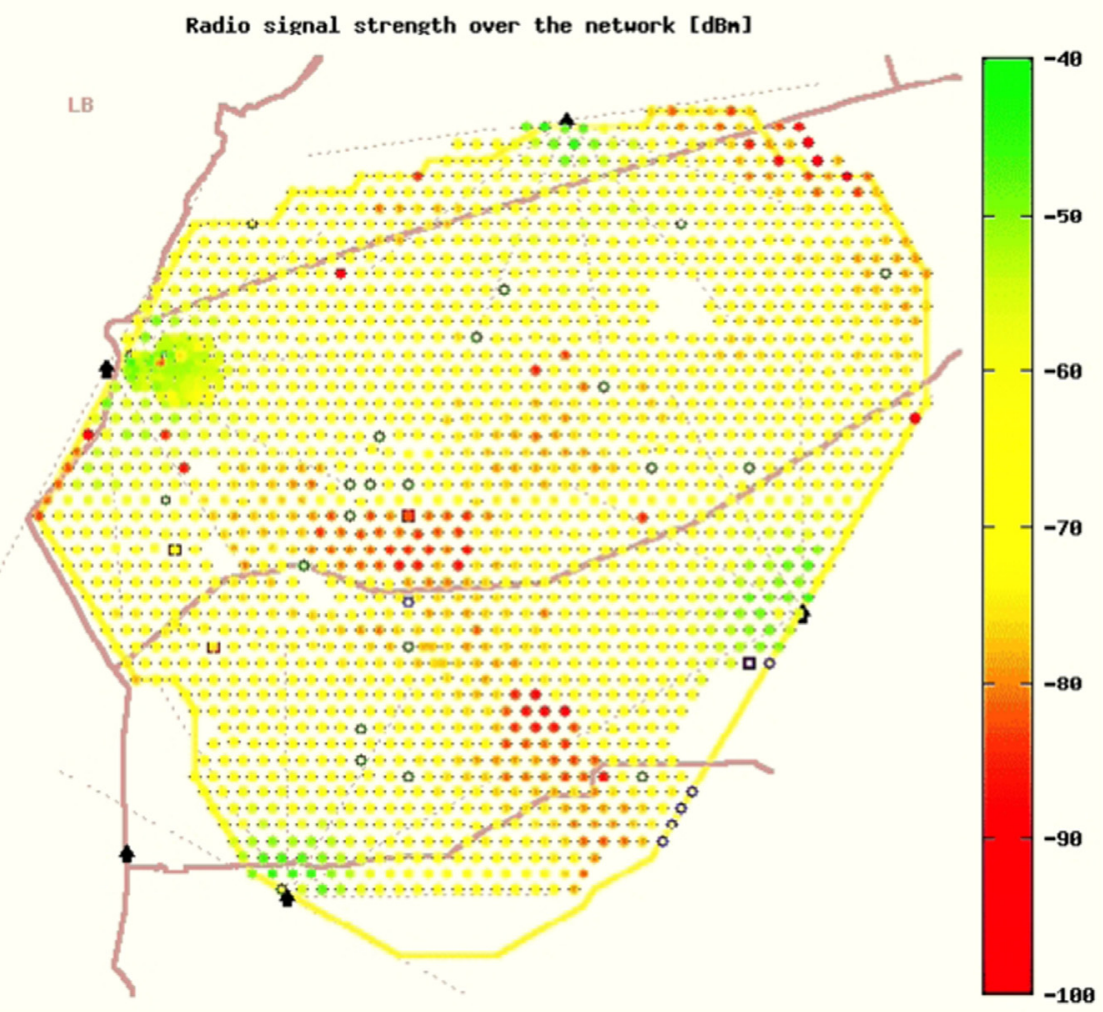

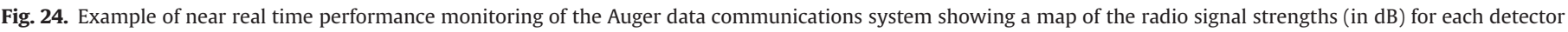
in the array.

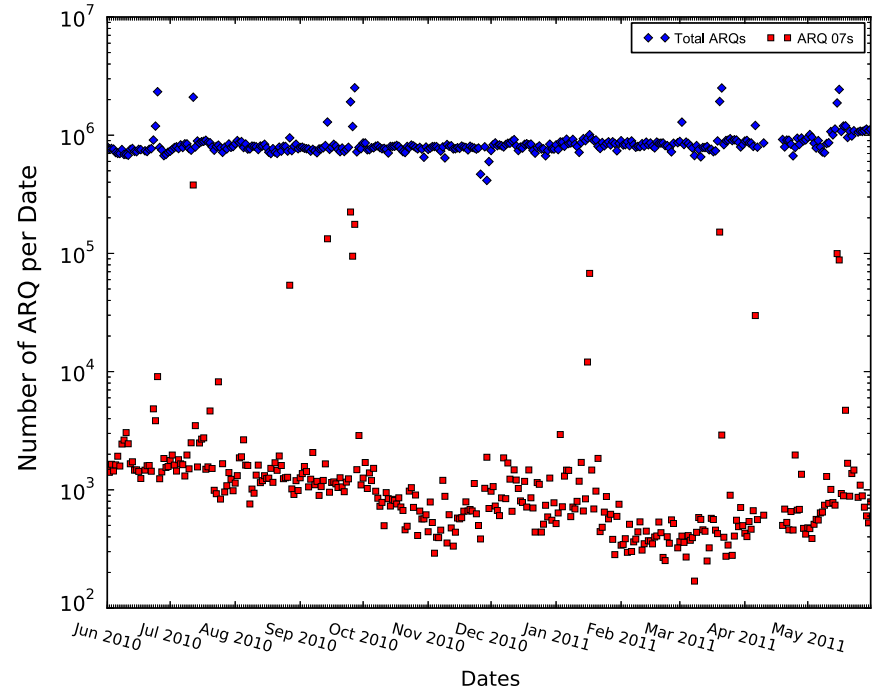

Fig. 25. Example of benchmark long term performance monitoring of the Auger data communications system showing the daily rate of ARQs over a period of 12 months from June 2010 to May 2011. Upper points indicate the rate of any ARQ; almost all of these result in successful retrieval of a lost or corrupt data packet. Lower points (ARQ7) correspond to packets which were lost after at least six attempts to retransmit.

\subsection{Data access}

The $\overline{\text { Off }}$ line framework includes two parallel hierarchies for accessing data: the detector description for retrieving data on conditions, including detector geometry, calibration constants, and atmospheric conditions, and an event data model for reading and writing information that changes for each event.

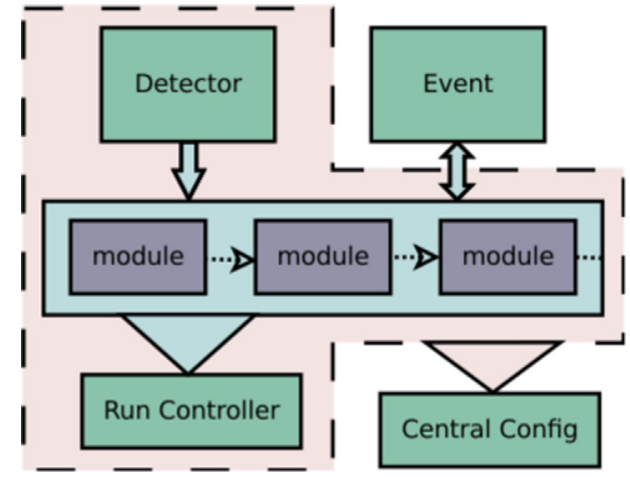

Fig. 26. General structure of the $\overline{\mathrm{Off}}$ line framework. Simulation and reconstruction tasks are encased in modules. Each module is able to read information from the detector description and/or the event, process the information, and write the results back into the event under command of a Run Controller. A Central Config object is responsible for handing modules and framework components their configuration data and for tracking provenance.

The detector description provides a unified interface from which module authors can retrieve conditions data. Data requests are passed by this interface to a back end comprising a registry of the so-called managers, each of which is capable of extracting a particular sort of information from a given data source. The manager mechanism allows for a simple interface to a potentially complex collection of different data sources and formats. The general structure of the detector description machinery is illustrated in Fig. 28.

The event data model contains the raw, calibrated, reconstructed and Monte Carlo information and serves as the backbone for communication between modules. The event is instrumented with a protocol allowing modules to discover its constituents at 


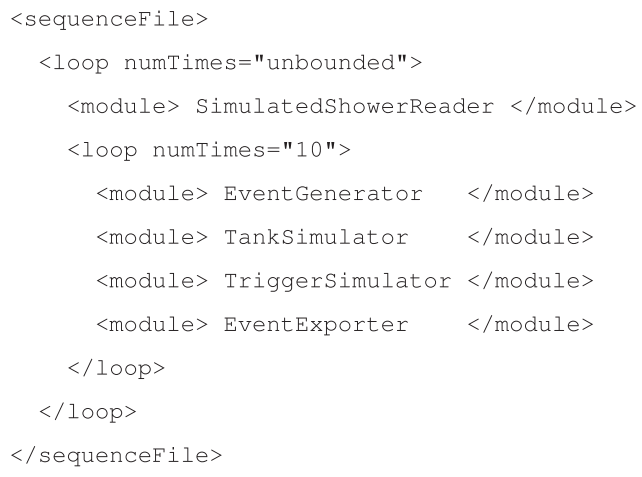

Fig. 27. A simplified example in which an XML file sets a sequence of modules to conduct a simulation of the surface array. <loop $>$ and $<$ module $>$ tags are interpreted by the run controller, which invokes the modules in the proper sequence. In this example, simulated showers are read from a file, and each shower is thrown onto the array in 10 random positions by an EventGenerator. Subsequent modules simulate the response of the surface detectors and trigger, and export the simulated data to file. Note that XML naturally accommodates common sequencing requirements such as nested loops.

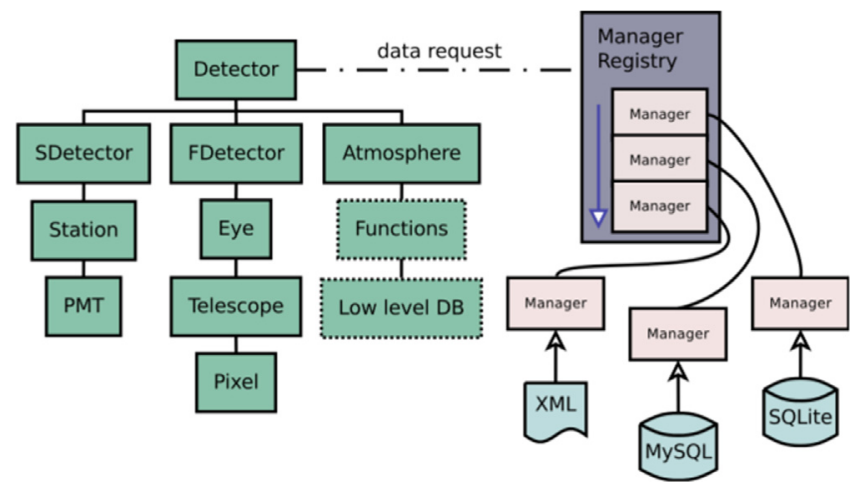

Fig. 28. Machinery of the detector description. The user interface (left) comprises a hierarchy of objects describing the various components of the Observatory. These objects relay requests for data to a registry of managers (right) which handle multiple data sources and formats.

any point in processing, and thereby determine whether the input data required to carry out the desired processing are available.

The transient (in memory) and persistent (on disk) event models are decoupled in order to avoid locking to a single provider solution for serialization, the process by which $\mathrm{C}++$ objects are converted to a form that can be written to disk. When a request is made to write event contents to file, the data are transferred from the transient event through a file interface to the persistent event, which is instrumented with serialization machinery. We currently use the input/output portion of the ROOT [57] toolkit to implement serialization. Various file formats are interpreted using the file interface, including numerous raw event and monitoring formats as well as the different formats employed by the AIRES [58], CORSIKA [59], CONEX [60] and SENECA [61] air shower simulation packages.

\subsection{Configuration}

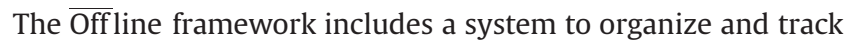
data used to configure the software for different applications as well as parameters used in the physics modules. A Central Config configuration tool (Fig. 26) points modules and framework components to the location of their configuration data, and creates Xerces-based [62] XML parsers to assist in reading information from these locations. We have wrapped Xerces with our own interface which provides ease of use at the cost of somewhat reduced flexibility, and which also adds functionality such as automatic units conversion and casting to various types, including commonly used containers.

The Central Config keeps track of all configuration data accessed during a run and stores them in an XML log file, which can subsequently be used to reproduce a run with an identical configuration. This allows collaborators to easily exchange and use configuration data for result comparisons. The logging mechanism is also used to record the versions of modules and external libraries which are used for each run.

Syntax and content checking of the configuration files is afforded through W3C XML Schema [63] standard validation. Schema validation is used not only for internal configuration prepared by framework developers, but also to check the contents of physics module configuration files. This approach reduces the amount of code users and developers must prepare and supports very robust checking.

\subsection{Utilities, testing and quality control, and build system}

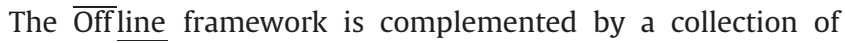
utilities, including an XML parser, an error logger and various mathematics and physics services. We have also developed a novel geometry package which allows the manipulation of abstract geometrical objects independent of coordinate system choice. This is particularly helpful for our applications since the Observatory comprises many instruments spread over a large area and oriented in different directions, and hence possesses no naturally preferred coordinate system. Furthermore, the geometry package supports conversions to and from geodetic coordinates based on a reference ellipsoid.

As in many large scale software development efforts, each low level component of the framework is verified with a small test program, known as a unit test. We have adopted the CppUnit [64] testing framework as an aid in implementing these tests. In addition to unit tests, a set of higher level acceptance tests has been developed which is used to verify that complete applications continue to function as expected, within some tolerance, during ongoing development. We employ a BuildBot system [65] to automatically compile

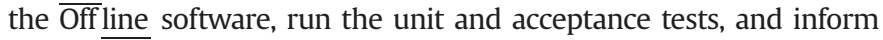
developers of any problems each time the code is modified.

The Off line build system is based on the CMake [66] crossplatform build tool, which has proven sufficient for this project. In

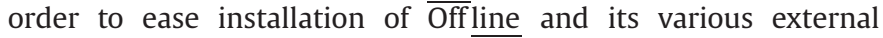
dependencies, we have prepared a tool known as APE (Auger Package and Environment) [67]. APE is a python-based dependency resolution engine, which downloads the external packages required for a particular application, builds them in whatever native build system applies for each package, and sets up the user's environment accordingly. APE is freely available, and has been adopted by other experiments, including HAWC, NA61/SHINE and JEM-EUSO.

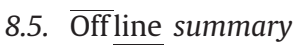

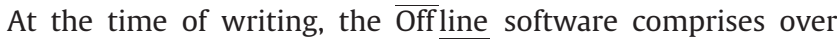
350,000 lines of code, corresponding to some 95 person years of effort, according to the Constructive Cost Model [68]. The framework has been enhanced for simulation and reconstruction of the Observatory extensions discussed in Sections 13.1 and 13.2 and for the radio research program described in Section 16.1, for which

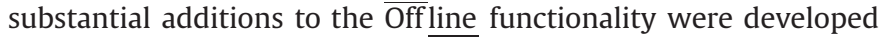
[69]. The code is available under an open source BSD license upon request. Other experiments have adopted portions of the $\overline{\text { Off line }}$ code, including Tunka-Rex [70], HAWC [71], JEM-EUSO [72], CODALEMA [73] and NA61/SHINE [74,75]. 


\section{Atmospheric monitoring}

The Observatory makes use of the atmosphere as a giant calorimeter. This motivated the selection of a site with generally good viewing conditions and the implementation of an extensive program to monitor the troposphere above the site. A detailed knowledge of the atmosphere is required for the accurate reconstruction of air showers observed by the FD [76-79] and for the accurate estimation of the exposure of the detectors [37].

The atmospheric state variables, including temperature, pressure and humidity, are needed to assess the longitudinal development of extensive air showers $[77,80]$ as well as the amount of the isotropically emitted fluorescence light induced by the air showers [81-84]. The SD observations are altered by different atmospheric conditions [85]. Varying air densities close to the ground modify the Molière radius affecting the lateral distribution of the electromagnetic component of the extensive air shower (EAS). Varying air pressure affects the trigger probability and the rate of events detected above a fixed energy. Furthermore, the atmospheric state variables are used to determine the Rayleigh (pure molecular) scattering of the fluorescence and Cherenkov light. Installations for recording local conditions of the state variables are described in Section 9.1.

Aerosols and clouds represent the most dynamic monitoring and calibration challenges at the Observatory. The optical transmission properties of the atmosphere, including the vertical aerosol optical depth profile $\tau_{\text {aer }}(h)$, have to be measured across the Observatory during FD data taking. In the air shower reconstruction, the atmospheric transmission between the FD and an air shower must be taken into account to properly reconstruct the light generated along the shower axis from the light recorded at the telescope(s) $[76,79]$. Moreover, Cherenkov light induced by the air showers is also detected with the FD and needs to be reconstructed as a function of atmospheric conditions at the time of the event. Installations dedicated for determining the optical scattering and absorption behavior of the atmosphere in the field of view are described in Section 9.2 and those for identifying and determining clouds and the general extinction above the Observatory in Section 9.3 .

An extensive system of atmospheric monitoring devices has been installed (Fig. 29). The types of measurements possible with these instruments are listed in Table 2.

\subsection{Installations for atmospheric state variables}

\subsubsection{Ground-based weather stations}

The Auger Collaboration operates several weather stations, as indicated in Fig. 29. Some of these stations are used for operational control of the nearby installations. The data from the weather stations at each FD site and at the CLF additionally serve as atmospheric ground information in several parts of the air shower reconstruction. Typically, those data are transferred via the central campus in Malargüe, processed and stored in our databases for atmospheric monitoring information (cf. Section 9.5) within a couple of days.

The weather stations are commercial products ${ }^{4}$ equipped with temperature, pressure, humidity, and wind speed sensors recording data every $5 \mathrm{~min}$. The stations at FD buildings Los Leones and Coihueco and at the laser facilities are additionally equipped with a sensor for wind direction. Formerly at the Balloon Launching Station (BLS) site and now at the AERA site (cf. Section 16.1), the weather station serves as a base unit for an electric field meter. The values of the electric field are recorded every second for

\footnotetext{
${ }^{4}$ Campbell Scientific, http://www.campbellsci.com.
}

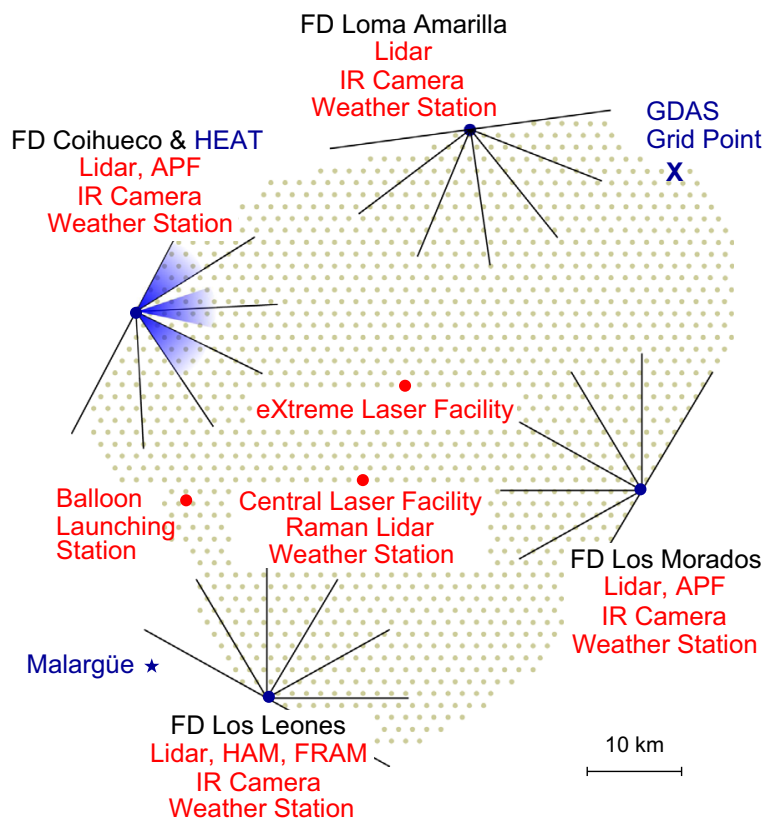

Fig. 29. Schematic overview of the atmospheric monitoring devices installed at the Pierre Auger Observatory. At each FD site, there is a lidar station, a ground-based weather station, and an infrared camera for cloud cover detection. In addition, there are devices for measuring the Aerosol Phase Function (APF) at FD Coihueco and Los Morados, a Horizontal Attenuation Monitor (HAM) at FD Los Leones, and a ph(F)otometric Robotic Atmospheric Monitor (FRAM) also at Los Leones. A steerable backscatter elastic lidar system is installed at each of the 4 FD sites to measure aerosols and the positions of clouds near each site. At central positions within the surface detector array, two laser facilities are installed (CLF and XLF). These instruments, together with the FD, are used to measure $\tau_{\text {aer }}(h)$ in the line of sight of each FD telescope 4 times per hour. In April of 2013 the CLF was upgraded with a Raman lidar receiver. Near the western boundary of the array, the Balloon Launching Station (BLS) was assembled together with a weather station as a base unit for an electric field meter. From this launch station, the weather balloons were typically carried across the entire array by westerly winds.

lightning and thunderstorm detection which is particularly important for the radio detection technique.

\subsubsection{Balloon Launching Station}

For a proper reconstruction of the fluorescence telescope signals, not only are ground-based atmospheric data needed, but also atmospheric profiles of the state variables temperature, pressure, and humidity up to about $20-25 \mathrm{~km}$ a.s.l. [77,80-82,86]. From these directly measured values, the derived quantities air density and atmospheric depth are calculated. The program of launching meteorological radiosondes attached to helium filled weather balloons was started at the Observatory site in August 2002. After 331 successfully measured profiles, the routine operation was terminated in December 2010 [78] and then replaced by the meanwhile validated GDAS data. During the first years, campaigns of about three weeks with an average of nine launches per campaign were done roughly three times a year. The starts of the soundings were usually placed at some FD buildings, mostly at Los Leones and Coihueco. In 2005, a dedicated BLS, cf. Fig. 29, was installed at a suitable position to optimally cover the large area above the surface detector array and in the field of view of the FD telescopes by the weather balloons. From this fully equipped station, more regular launches could be managed, in particular during the night. Between July 2005 and March 2009, roughly one launch was performed about every five days. Between 2009 and 2011, the program was part of the rapid atmospheric monitoring system of the Pierre Auger Observatory (see Section 9.4). A radiosonde launch was triggered shortly after the detection of 
Table 2

Atmospheric measurements performed and the instruments that are used.

\begin{tabular}{llll}
\hline Category & Variable & Frequency & Instrument(s) \\
\hline \multirow{2}{*}{ State } & $\begin{array}{l}\text { At ground: pressure, temp., wind, humidity } \\
\text { Profile: pressure, temp., humidity }\end{array}$ & $\begin{array}{l}5 \mathrm{~min} \\
3 \mathrm{~h}\end{array}$ & $\begin{array}{l}\text { Weather stations } \\
\text { GDAS }\end{array}$ \\
& & & \\
\multirow{2}{*}{ Aerosols } & Vert. optical depth $(z)$ & Hourly & CLF, XLF + FD \\
& Phase function & Hourly & 2 APF units \\
& Ångström coefficient & Hourly & FRAM (HAM) \\
Clouds & Presence in FD pixels & 15 min & 4 cloud cameras \\
& Behind FD sites & 15 min & 4 lidar stations \\
& Along select tracks & Avg. 1/night & FRAM, lidar \\
& Above CLF/XLF & Hourly & CLF, XLF + FD \\
\hline
\end{tabular}

a Atmospheric model developed at the National Centers for Environmental Prediction, operated by NOAA; provided via READY - Real time Environmental Applications and Display sYstem.

particularly interesting air showers such as very energetic events. Since 2011, the BLS is used for dedicated measurement campaigns.

The radiosondes and the receiver station are standard meteorological products ${ }^{5}$ providing data on the temperature, pressure, humidity, and GPS position including altitude. Typically, a set of measurement values is recorded every $2-8 \mathrm{~s}$. The upper limit of the profile is given by the height of the burst of the weather balloon, typically at about $23 \mathrm{~km}$, with a few balloons reaching a maximum altitude of $27 \mathrm{~km}$.

Based on the locally measured atmospheric profiles, monthly models of atmospheric conditions at the Pampa Amarilla were derived in December 2008 [79,78]. The monthly models are also compiled for application in air shower simulations like CORSIKA [59]. Finally, these measurements were used to validate the utility of data from the Global Data Assimilation System (GDAS) for the purpose of air shower reconstruction at the Pierre Auger Observatory [77]. GDAS is the result of forecasts which are run several times per day and are based on meteorological measurements from all around the world. The data are available in 3-hourly, global, $1^{\circ}$ latitude-longitude $\left(360^{\circ}\right.$ by $180^{\circ}$ ) datasets. The position of the chosen GDAS grid point is marked in Fig. 29. Each GDAS dataset consists of surface data together with data for 23 constant pressure levels reaching up to about $26 \mathrm{~km}$. For the purpose of the Auger Observatory, mainly the information for temperature, pressure and humidity are used. These GDAS data have been compared with local radio soundings and the records from the ground-based weather stations. The agreement of the locally recorded data with the GDAS data for the given grid point is good enough for the application in air shower physics, cf. Fig. 30. The variation for temperature is below $\pm 1 \mathrm{~K}$ for altitudes between ground and about $20 \mathrm{~km}$ a.s.l., for pressure, the variation is below $0.5 \mathrm{hPa}$ at worst, but for most of the altitude range well below $0.3 \mathrm{hPa}$. Even the vapor pressure is well below $0.3 \mathrm{hPa}$ except for the data point closest to ground where the difference goes up to $1.0 \mathrm{hPa}$. After validating the utility of the GDAS data for the site of the Pierre Auger Observatory [77], advanced monthly models were compiled from GDAS data for this location for air shower simulations and reconstructions $[78,87]$.

\subsection{Installations for atmospheric transmission}

The transition of fluorescence light, induced by extensive air showers, in the atmosphere is reduced by absorption and scattering of the UV photons. For a correct reconstruction of the energy

\footnotetext{
5 http://www.graw.de
}

deposit of an extensive air shower in the atmosphere, the attenuation of the fluorescence photons has to be known for the time of the air shower.

The attenuation of light in the wavelength range of interest here is dominated by scattering rather than by absorption. The scattering of photons in air can be described analytically from molecular scattering theory. Once the vertical profiles of atmospheric temperature, pressure, and humidity are known, the molecular transmission factor $T_{m o l}(\lambda, s)$ is a function of the total wavelength-dependent Rayleigh scattering cross-section along the line of sight $s$. The scattering of photons by aerosols can be described by Mie scattering theory, but for real conditions with strongly varying shapes and amounts of aerosols, local measurements are needed. The knowledge of the aerosol transmission factor $T_{a e r}(\lambda, s)$ depends on frequent field measurements of the vertical aerosol optical depth $\tau_{a e r}(h)$, the integral of the aerosol extinction $\alpha_{a e r}(z)$ from the ground to a point at altitude $h$ observed at a given elevation angle.

\subsubsection{Aerosol optical depth profiles and clouds: $C L F$ and XLF}

Laser tracks from the CLF and XLF (Section 4.4.2) are recorded by the $4 \mathrm{FD}$ sites. They are used to obtain hourly measurements of the aerosol optical depth profiles [88] that are used in the reconstruction of each FD air shower event. Sets of 50 vertical shots are measured every $15 \mathrm{~min}$ by the FD telescopes throughout each night. The polarization of the CLF beam is randomized so that the amount of light scattered out of the beam is azimuthally symmetric about the beam axis. The CLF data sample began in 2004 and that for the XLF in 2009. These samples contain more than 1.5 million laser shots corresponding to more than 4 million tracks in the FD telescopes. To obtain the aerosol optical depth profile for each FD site, two techniques are used: the 50-shot averages are compared to averages collected under clear conditions (Data Normalized Analysis [88]), and to simulations generated with different aerosol attenuation conditions (Laser Simulation Analysis [88]). Data from the Aerosol Phase Function monitors (next paragraph) can be used to cross-check the clear reference periods. The technique is independent of the absolute photometric calibrations of the lasers and of the FD, and provides essential information in the reconstruction of hybrid and FD data. In Fig. 31, data from the vertical aerosol optical depth at $3.5 \mathrm{~km}$ above the fluorescence telescopes are shown.

The analysis of the light profile of laser shots, as detected by the FD, also provides measurements of the heights of clouds directly above the CLF or XLF [88]. 

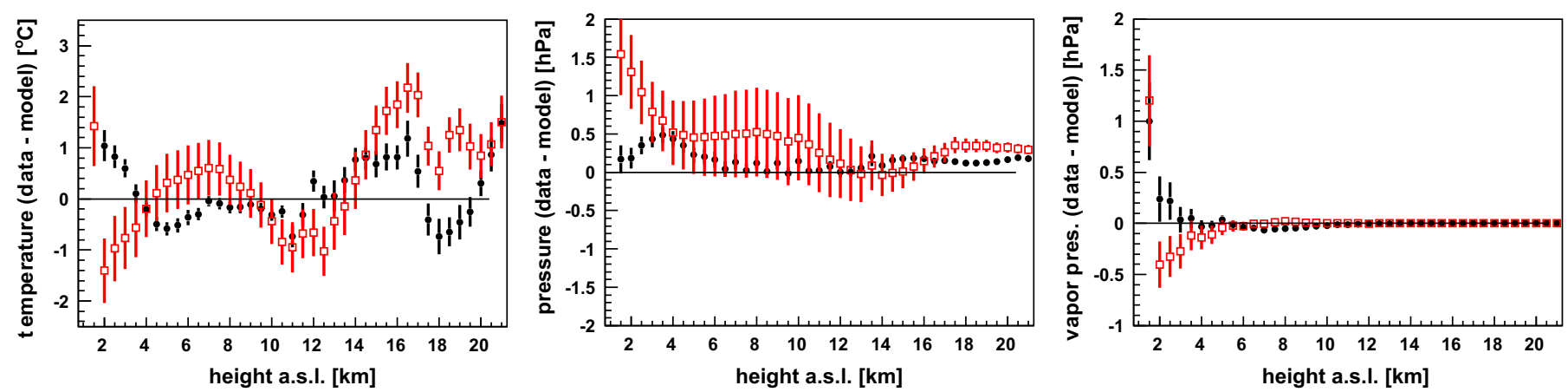

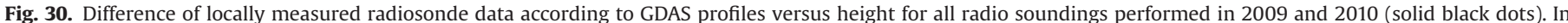

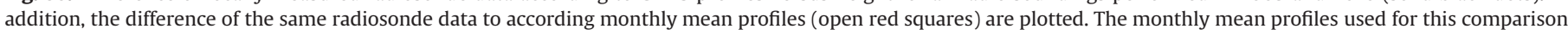
are those developed at the end of 2008, representing a totally independent dataset of local records than the plotted radiosonde data [77].
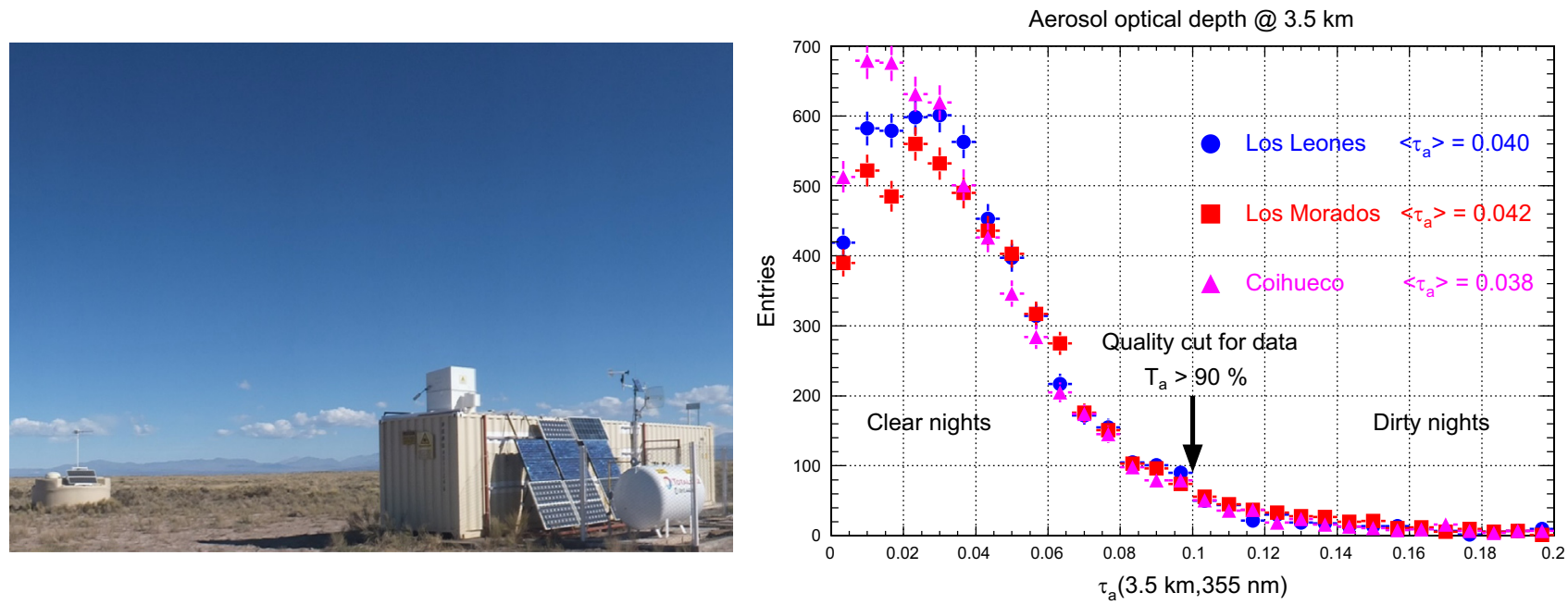

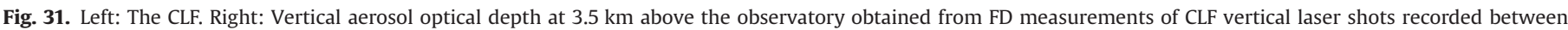
January 2004 and December 2010. Data from 3 fluorescence stations are shown and the quality cut between clear and dirty nights is indicated [89].

\subsubsection{Aerosol Phase Function Monitors}

The atmospheric scattering of both fluorescence and Cherenkov light from extensive air showers occurs over a range of scattering angles. The scattering angular distribution (phase function) can be estimated analytically for the atmospheric molecular component. For the aerosol component this function depends on the size and shape of the aerosols [76]. The scattering function is characterized in situ to implement a suitable parametrization of the scattering behavior by the air shower reconstruction.

The Aerosol Phase Function Monitors use a collimated xenon flash lamp to direct light between 350 and $390 \mathrm{~nm}$ horizontally across the FD field of view at Coihueco and Los Morados. The FD measures the light as a function of scattering angle (30-150 $)$. An analysis including data over several years revealed that a parameterization as

$P_{a}(\theta)=\frac{1-g^{2}}{4 \pi} \cdot\left(\frac{1}{\left(1+g^{2}-2 g \cos \theta\right)^{3 / 2}}+f \frac{3 \cos ^{2} \theta-1}{2\left(1+g^{2}\right)^{3 / 2}}\right)$

describes the aerosol scattering at the Observatory site reasonably well. The first term is a Henyey-Greenstein function [90], corresponding to forward scattering, and the second term accounts for the peak at large $\theta$ typically found in the angular distribution of aerosol-scattered light [91]. The quantity $g$ describes the asymmetry of scattering, and $f$ determines the relative strength of the forward and backward scattering peaks. An average value of the phase function asymmetry parameter $g$ of $0.56 \pm 0.10$ is used in the Auger air shower analysis for nights with Mie scattering. For clear nights without any aerosols, $g$ is set to zero. To also allow for very small aerosol content during almost clear nights, causing only small asymmetries in the phase function, an uncertainty of 0.2 is estimated and attached to the value of $g$ equals zero.

\subsubsection{Horizontal Attenuation Monitor}

The wavelength dependence of the aerosol attenuation is modeled by a falling power law with an exponential parameter $\gamma$. The value of $\gamma$ varies inversely with the typical size of the aerosol particles. In the limit of clean air, $\gamma \approx 4$. At the Observatory, $\gamma$ is obtained by the Horizontal Attenuation Monitor (HAM). The HAM consists of a high intensity discharge lamp installed close to the FD site at Coihueco. Light from this lamp is measured by a filtered CCD camera at the Los Leones FD site, about $45 \mathrm{~km}$ away [76]. Total horizontal atmospheric attenuation is measured over this path at five wavelengths between 350 and $550 \mathrm{~nm}$. The data indicate that the atmosphere of the Observatory is quite desert-like with weak wavelength dependence. An average $\gamma$ of about 0.7 with an RMS of 0.5 is used as a parameter in the air shower reconstruction.

\subsection{Installations for clouds and extinction}

\subsubsection{Cloud identification}

The presence of clouds can alter the observed optical signatures of an EAS and reduce the aperture of the FD. Clouds can attenuate 
or block light from an air shower, producing a dip in the longitudinal profile observed by the FD. Conversely, if a shower passes through a cloud layer, the cloud can enhance the scattering of the intense Cherenkov light beam, producing a bump [92].

The Observatory uses measurements from infrared cameras and lidar systems and FD measurements of CLF and XLF tracks to detect clouds. A cloud is warmer than the surrounding atmosphere and produces an infrared signal that depends on the cloud temperature and emissivity (or optical depth). An infrared camera (Raytheon ControlIR 2000B) mounted on a pan-and-tilt scanning platform, operates at each FD building. The cameras are sensitive in the $7-13 \mu \mathrm{m}$ band, appropriate for the peak of the blackbody radiation from thick clouds, but unfortunately also sensitive to water emission bands even in clear sky. Every 5 min each camera scans the field of view (FOV) of the telescopes, and every 15 min the entire hemisphere is imaged. The raw FOV images are converted into a binary image (cloudy/clear) after a series of image processing steps designed to remove camera artefacts and account for the expected elevation angle dependence of the clear sky intensity [93]. These data are then mapped onto FD pixel directions to indicate the presence or absence of clouds in each FD pixel. At the time of writing, the Raytheon IR cameras are being replaced by Xenics Gobi 384 radiometric IR cameras to improve image processing and reduce cable maintenance.

Data from the Geostationary Operational Environmental Satellites (GOES) are also being analyzed [94]. GOES instruments provide radiance data in one visible and four infrared bands from which brightness temperatures are derived. The GOES-12 imager instrument covers the area of the Pierre Auger Observatory every $30 \mathrm{~min}$. Cloud probability maps with a grid of $2.4 \mathrm{~km}$ by $5.5 \mathrm{~km}$ pixel size are derived for the area of the Observatory. Cross-checks of cloud identification as derived from GOES measurements for the pixels viewing the CLF and as derived from FD measurements of CLF vertical tracks show a reliable correlation.

\subsubsection{Clouds and aerosols: FD lidar stations}

Four elastic lidars, installed next to each FD station, are used to measure cloud cover, cloud height and aerosols [53,95]. Each lidar has a Nd:YLF laser that produces $0.1 \mathrm{~mJ}$ pulses at a wavelength of $351 \mathrm{~nm}$. Three $80 \mathrm{~cm}$ mirrors and a $20 \mathrm{~cm}$ mirror collect the backscattered light which is measured with Hamamatsu R7400U photomultipliers. A UG-1 optical filter reduces background light. The lasers are operated remotely at a repetition rate of $333 \mathrm{~Hz}$. Thousands of pulses are averaged by analog and photon counting readout systems. The two traces from these parallel readout paths are then combined to cover from $200 \mathrm{~m}$ up to $25-30 \mathrm{~km}$.

The lidars are steerable and perform discrete and continuous scanning patterns automatically during the FD operation. To avoid interference with FD data collection, most of the scanning shots are aimed outside the FD field of view. Two exceptions are horizontal shots fired in the direction of the CLF to measure ground level aerosol horizontal attenuation length and shootthe-shower scans to probe the detector shower plane shortly after especially interesting cosmic ray candidates have been observed.

Long term measurements from these lidars find a mean aerosol extinction length at ground level of $0.028 \mathrm{~km}$, which indicates that the atmosphere at the site is quite clear. Furthermore, it can be derived that about $62 \%$ of the FD data taking time is quite clear with a mean cloud cover of less than $20 \%$.

\subsubsection{FRAM}

The ph(F)otometric Robotic Atmospheric Monitor (FRAM) is an optical telescope ( $0.3 \mathrm{~m}$ diameter mirror) that measures starlight to determine the wavelength dependence of Rayleigh and Mie scattering. It is also used to make automatic observations of light curves of optical transients associated with gamma ray bursts [96].

FRAM was installed at the site of the Los Leones fluorescence detector in 2005. Since the end of 2009, it has been part of the rapid atmospheric monitoring program, cf. Section 9.4. Because the FRAM is a passive instrument, it can operate in the field of view of the FD. A photometric observation of selected standard (i.e., non-variable) stars has been supplemented with a photometric analysis of CCD images since 2011 [97,79]. A wide field camera is used to measure the atmospheric extinction along the shower detector plane. Its field of view is $240^{\prime}\left(4^{\circ}\right)$ in azimuth (aligned with right ascension) and $160^{\prime}\left(2.67^{\circ}\right)$ in elevation (aligned with declination). This instrument is a Finger Lake Instrumentation (FLI) MaxCam CM8 with Carl Zeiss Sonnar $200 \mathrm{~mm} f / 2.8$ telephoto lens. A second, narrow field camera is used to calibrate the images of the wide field camera. Before June 2010, this camera was also a FLI instrument, but was exchanged for a Moravian Instruments CCD camera G2.

\subsection{Rapid monitoring}

In 2009, the atmospheric monitoring program was upgraded [79] to probe the shower FD detector plane with the FRAM and lidar systems a few minutes after the FD detected any extremely high energy EAS or an EAS with an unusual longitudinal profile. All atmospheric subsystems involved use individual and modifiable trigger settings to identify these kind of FD detected air showers. The motivation was to check for clouds or aerosol layers that might distort the observed profile. Between March 2009 and December 2010, a weather balloon was also released from the BLS site to measure the pressure, temperature and humidity profiles within a few hours of the EAS detection above the Auger array with an energy above $10^{19} \mathrm{eV}$. During this period, $100 \mathrm{FD}$ events were triggered for a weather balloon launch. Some of the triggers were received while a radiosonde was already in flight, due to the tendency of high-quality, high-energy observations to cluster during very clear, cloudless nights. Thus, 62 triggers were covered by 52 flights. The remaining triggers were lost due to technical issues such as hardware failures at the BLS, problems with the transmission of the text message to the technician who needed to launch the weather balloon, or other failures in the radiosonde flights.

\subsection{Atmospheric databases}

The atmospheric monitoring data are organized into MySQL databases that are accessed by the Auger offline analysis package for air shower reconstruction, condition assessment, and aperture estimates.

The cloud information includes the IR cloud camera distributions mapped onto the FD pixels and the hourly measurement of the fraction of the sky covered by cloud measured by the lidars. An hourly cloud coverage below $20 \%$ is required for hybrid events to be used in the analysis of the mass composition and energy spectrum of the cosmic rays $[21,38]$.

The aerosol optical database contains, for each FD site, hourly $\tau_{\text {aer }}(h)$ profiles in $200 \mathrm{~m}$ steps and derived from CLF or XLF laser shots. FD data used for cosmic ray publications is required to be collected during hours with $\tau_{\text {aer }}(3 \mathrm{~km}$ a.g.l. $)<0.1$. FD data recorded during hours without $\tau_{\text {aer }}(h)$ profiles due to extremely poor viewing conditions or technical problems are not used for publications. This database spans nearly 10 years.

The molecular database contains the atmospheric state variables measured on the ground by the weather stations and the vertical profiles derived from BLS weather balloons and more recently from the Global Data Assimilation System, provided from 
NOAA's ${ }^{6}$ National Centers for Environmental Prediction (NCEP) [77]. These atmospheric data are available for a position close to the Auger array in $3 \mathrm{~h}$ intervals. Data updates can be obtained once per week and are filled automatically into the Auger atmospheric monitoring database. Such atmospheric state variables are applied as standard in the air shower reconstruction, mainly during the description of the fluorescence light emission and the Rayleigh scattering of that light on its path between emission point at the air shower and the fluorescence telescopes, since mid-2011.

\subsection{Interdisciplinary atmospheric measurements}

Through its secondary role as an Earth observatory, the interdisciplinary science program of the Observatory is quite extensive [98]. For example, the FD has turned out to be the world's best detector for measuring atmospheric transient luminous events known as ELVES (see Section 14.2) [99].

To characterize local aerosol particles, an Andersen-Graseby 240 dichotomous sampler was installed at the Coihueco FD building for 6 months. The particles collected were studied to determine their sizes and shapes. Low aerosol concentrations were observed during the winter with an increasing concentration in spring. An elemental composition analysis was also performed [100].

The HYbrid Single Particle Lagrangian Integrated Trajectory (HySPLIT) model (developed from NOAA) that estimates the trajectories of air mass displacements was used to study the source of aerosols at the Observatory. A possible correlation was observed between clear conditions at the Observatory, determined from measured $\tau_{\text {aer }}(h)$ profiles, and air mass sources at the Pacific ocean [101].

\section{Hybrid event reconstruction}

The hybrid reconstruction is based on fluorescence detector data with additional timing information from the surface detector. In the following, the individual reconstruction steps will be described.

\subsection{Pulse reconstruction at the FD}

At the beginning of the reconstruction, the baseline is subtracted from the ADC trace of each pixel and the background noise is estimated from the variance of the ADC signals at early time bins that are free from any shower signal. Each ADC count is then converted to photons at the aperture using the calibration constants obtained from the drum and relative calibrations.

Subsequently, each triggered FD pixel is searched for a shower signal by scanning the signal trace for pulse start and stop times that maximize the signal to noise ratio. Only pulses with a signal to noise ratio $\geq 5$ are considered in the geometrical reconstruction.

The pulse time of the $i$ th pixel is given by the centroid time ("signal weighted time") of all trace bins belonging to the pulse:

$t_{i}=\frac{\sum \tau_{k}^{i} s_{k}^{i}}{q_{i}}$

where the sum runs over the time bins defined by the aforementioned signal to noise maximization. $\tau_{k}$ and $s_{k}$ are the time and charge of the kth ADC bin, respectively, and the pixels' integrated signal is given by

$q_{i}=\sum s_{k}^{i}$

\footnotetext{
${ }^{6}$ National Oceanic and Atmospheric Administration
}

The uncertainties of $q_{i}$ and $t_{i}$ are obtained by propagating the noise variance and Poissonian photoelectron fluctuations into Eqs. (2) and (3).

\subsection{Shower detector plane}

The shower detector plane is the plane containing the shower axis and the triggered fluorescence telescope. It can be reconstructed from the data of a telescope by minimizing

$S=\frac{1}{\sum_{i} q_{i}} \sum_{i} q_{i}\left(\frac{\frac{\pi}{2}-\arccos \left(\vec{p}_{i} \cdot \vec{n}_{\perp}^{\mathrm{SDP}}\right)}{\sigma_{\mathrm{SDP}}}\right)^{2}$

over all pulses $i$, with the two free parameters $\theta_{\mathrm{SDP}}$ and $\phi_{\mathrm{SDP}}$ to define the vector $\vec{n}_{\perp}^{\mathrm{SDP}}$ normal to the plane in spherical coordinates and the pixel pointing direction $\vec{p}_{i}$. The pointing uncertainty for the SDP fit, $\sigma_{\mathrm{SDP}}$, was determined to be $0.35^{\circ}$ by studying SDP fits of CLF laser shots with a known geometry. The normalization of the fit function $S$ to $\sigma_{\mathrm{SDP}}$ allows one to interpret it as a $\chi^{2}$ function and to derive the uncertainties of the SDP parameters from the $S+1$ contours.

\subsection{Hybrid time fit}

From the perspective of a telescope, the projection of a shower on the camera evolves along the SDP. Each pulse pixel can be associated with an angle $\chi_{i}$ along the SDP with respect to the horizontal axis at the telescope (see Fig. 32). The angular movement of the shower within the SDP in this representation is [102]

$t\left(\chi_{i}\right)=T_{0}+\frac{R_{\mathrm{p}}}{c} \tan \left(\frac{\chi_{0}-\chi_{i}}{2}\right)$.

To determine the three free parameters $T_{0}, R_{\mathrm{p}}$ and $\chi_{0}$ the minimum of the function

$\chi^{2}=\sum_{i} \frac{\left(t_{i}-t\left(\chi_{i}\right)\right)^{2}}{\sigma\left(t_{i}\right)^{2}}+\frac{\left(t_{\mathrm{SD}}-t\left(\chi_{\mathrm{SD}}\right)\right)^{2}}{\sigma\left(t_{\mathrm{SD}}\right)^{2}}$

has to be found [34,35]. The sum runs over all pulse pixels $i$ with the centroid pulse time $t_{i}$ and the associated uncertainty $\sigma\left(t_{i}\right)$, adding the additional SD station time $t_{\mathrm{SD}}$ with the uncertainty $\sigma\left(t_{\mathrm{SD}}\right)$. The shower front containing the surface detector station meets the (trial) shower axis at a point that would be seen at angle $\chi_{\mathrm{SD}}$, and $t\left(\chi_{\mathrm{SD}}\right)$ is the expected time when the shower center would pass that point.

An example of an event that has been observed by four telescopes is shown in Fig. 33. The individual four reconstructions of the geometry using the hybrid approach are indicated by black lines.

\subsection{Light collection}

The total light at the aperture as a function of time is obtained by adding the signals $s$ of the camera pixels $j$ at each time bin $i$. For this purpose, only the pixels with pointing directions that are within a certain angular distance $\zeta$ to the vector from the telescope to the shower center at time $i$ are included. $\zeta$ is chosen such that the signal to noise ratio is maximized. The light flux arriving at the detector in time bin $i$ is

$F_{i}=\frac{1}{A_{\mathrm{dia}}} \sum_{j=1}^{N_{\mathrm{pix}}} s_{i j}$

where the sum runs over all pixels $N_{\text {pix }}$ within $\zeta$ at time bin $i$ and $A_{\text {dia }}$ is the area of the diaphragm opening. 


\subsection{Profile reconstruction}

Once the geometry of the shower is known, the light collected at the aperture as a function of time can be converted to the energy deposited by the shower as a function of slant depth. For this purpose, the light attenuation from the shower to the detector needs

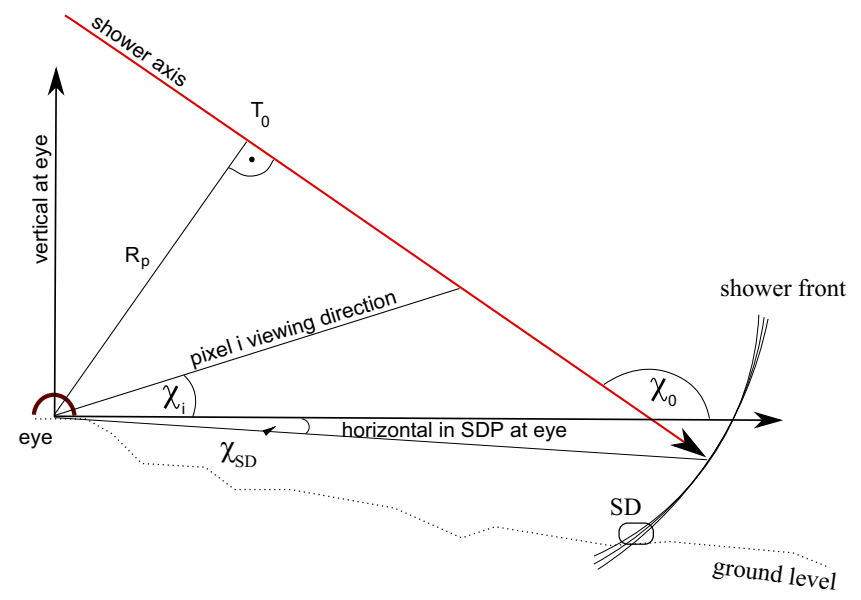

Fig. 32. Geometry of an air shower within the shower detector plane.

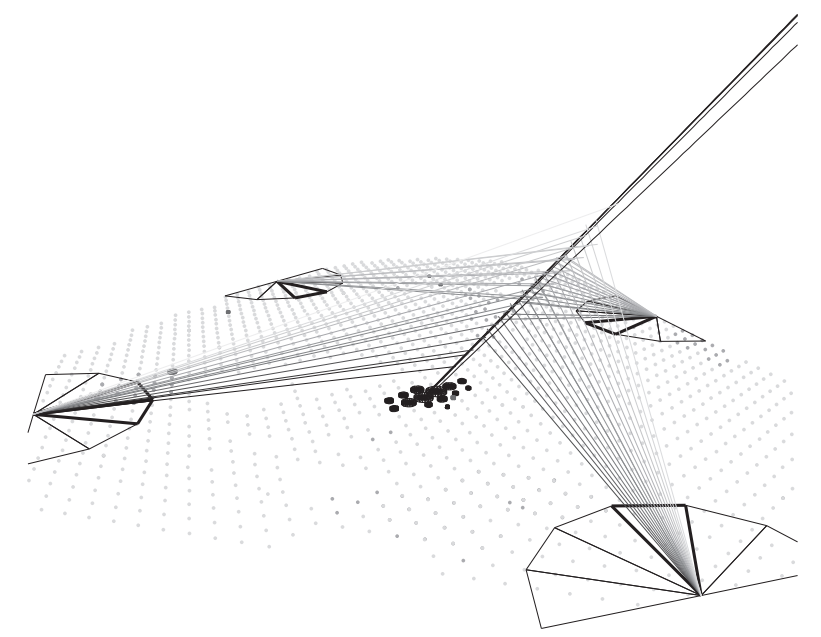

Fig. 33. Geometry reconstruction of an event observed by four telescopes and the surface detector.

(a)

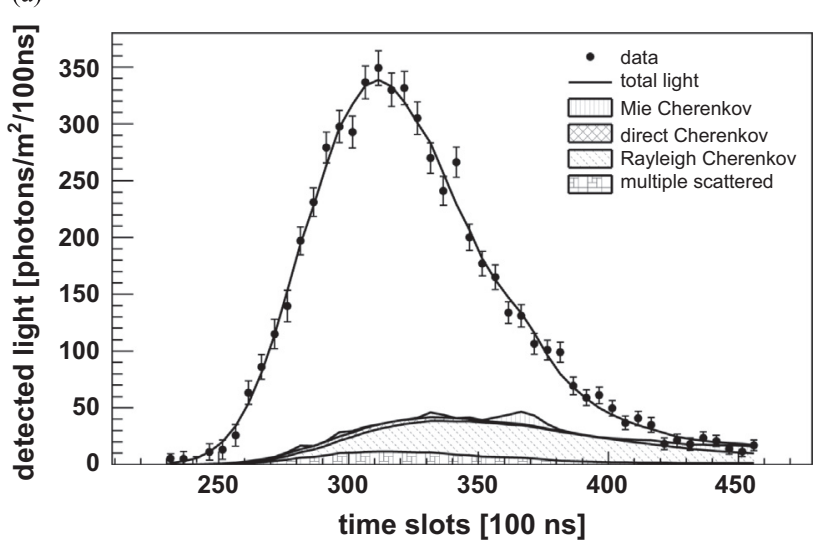

to be accounted for and all contributing light sources need to be disentangled: fluorescence light [81], direct and scattered Cherenkov light $[103,104]$ as well as multiply scattered light [105-107].

The proportionality between the fluorescence intensity and the energy deposit is given by the fluorescence yield. A good knowledge of its absolute value as well as its dependence on wavelength, temperature, pressure and humidity is essential to reconstruct the longitudinal profile. We use the most precise of the measurements available to date (cf. [108]) as provided by the Airfly Collaboration $[109,110]$.

The Cherenkov and fluorescence light produced by an air shower are connected to the energy deposit by a linear set of equations and therefore the shower profile is obtained by an analytic linear least squares minimization [111]. Due to the lateral extent of air showers, a small fraction of shower light is not contained within the optimal light collection area. To correct this, the universal lateral fluorescence [112] and Cherenkov light [113] distributions must be taken into account. The full longitudinal energy deposit profile and its maximum $(\mathrm{d} E / \mathrm{d} X)_{\max }$ at depth $X=X_{\max }$ are estimated by fitting a Gaisser-Hillas function [114]:

$f_{\mathrm{GH}}(X)=\left(\frac{\mathrm{d} E}{\mathrm{~d} X}\right)_{\max }\left(\frac{X-X_{0}}{X_{\max }-X_{0}}\right)^{\left(X_{\max }-X_{0}\right) / \lambda} e^{\left(X_{\max }-X\right) / \lambda}$

to the photoelectrons detected in the PMTs of the FD cameras. For this purpose, a log-likelihood fit is used in which the number of photoelectrons detected by the PMTs of the FD cameras is compared to the expectation from Eq. (8) after folding it with the light yields, atmospheric transmission, lateral distributions and detector response. The two shape parameters $X_{0}$ and $\lambda$ are constrained to their average values to allow for a gradual transition from a two- to a four-parameter fit depending on the observed track length and number of detected photons of the respective event (cf. [111]).

Finally, the calorimetric energy of the shower is obtained by integrating Eq. (8) and the total energy is estimated by correcting for the "invisible energy" carried away by neutrinos and high energy muons [115]. An example of the measured light at aperture and the reconstructed light contributions, and energy deposit profile is shown in Fig. 34(a) and (b).

\section{SD event reconstruction}

The reconstruction of the energy and the arrival direction of the cosmic rays producing air showers that have triggered the surface detector array is based on the sizes and times of signals registered from individual SD stations. At the highest energies, above $10 \mathrm{EeV}$, the footprint of the air shower on the ground extends over more than $25 \mathrm{~km}^{2}$. By sampling both the arrival times and the deposited

(b)

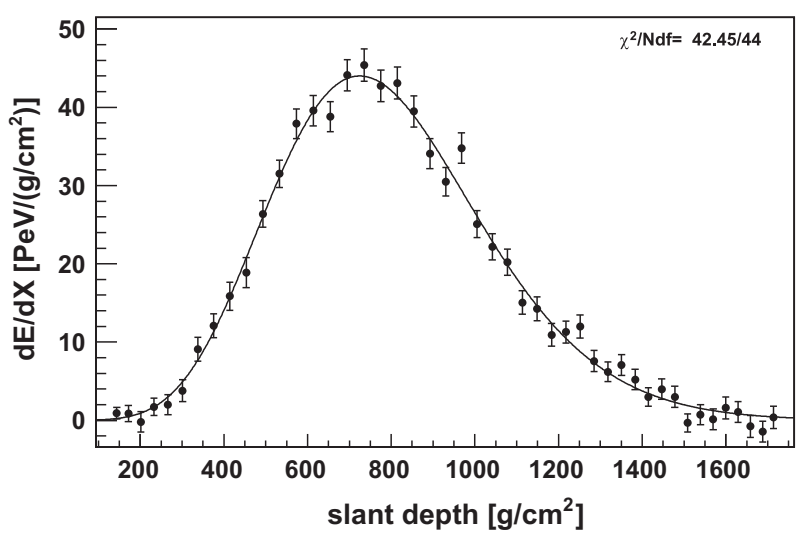

Fig. 34. Example of a reconstructed shower profile. (a) Light at aperture. (b) Energy deposit. 


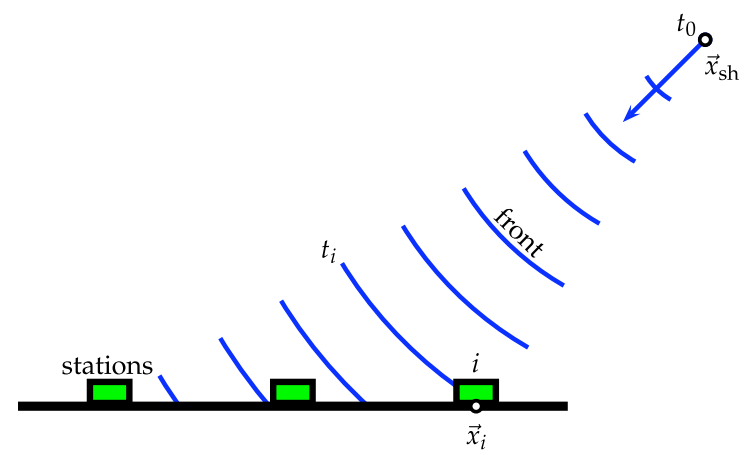

Fig. 35. Reconstruction of shower geometry: schematic representation of the evolution of the shower front.

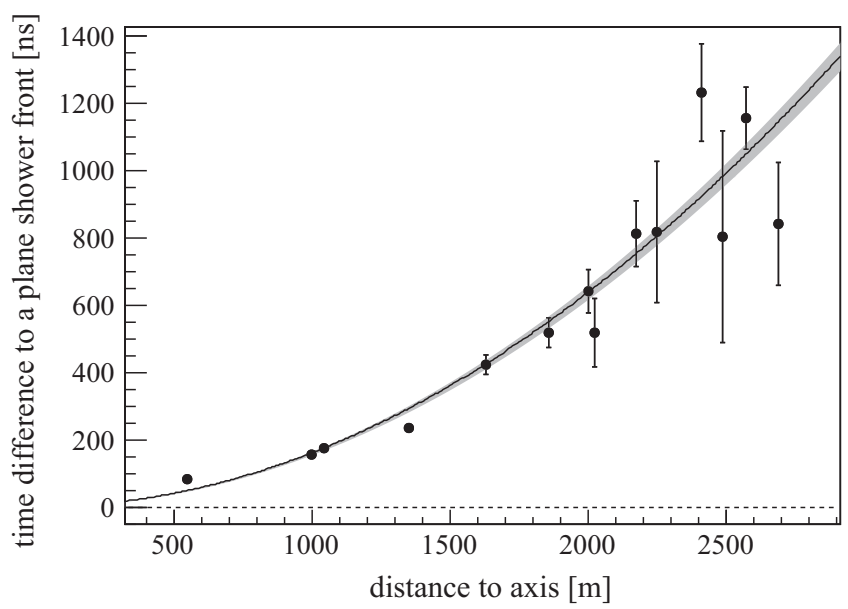

Fig. 36. Reconstruction of shower geometry: dependence of signal start times (relative to the timing of a plane shower front) on perpendicular distance to the shower axis. The shaded line is the resulting fit of the evolution model and its uncertainty.

signal in the detector array, the shower geometry, i.e., the shower core, the arrival direction of the incident cosmic ray, and the shower size can be determined.

\subsection{Event selection}

To ensure good data quality for physics analysis there are two additional off-line triggers. The physics trigger, T4, is needed to select real showers from the set of stored T3 data (see Section 6.3) that also contain background signals from low energy air showers. This trigger is mainly based on a coincidence between adjacent detector stations within the propagation time of the shower front. In selected events, random stations are identified by their time incompatibility with the estimated shower front. The time cuts were determined such that $99 \%$ of the stations containing a physical signal from the shower are kept. An algorithm for the signal search in the time traces is used to reject signals produced by random muons by searching for time-compatible peaks.

To guarantee the selection of well-contained events, a fiducial cut (called the 6T5 trigger) is applied so that only events in which the station with the highest signal is surrounded by all 6 operating neighbors (i.e., a working hexagon) are accepted. This condition assures an accurate reconstruction of the impact point on the ground, and at the same time allowing for a simple geometrical calculation of the aperture/exposure [36], important for, e.g., the spectrum analysis [38]. For arrival-direction studies a less strict cut can be used (5T5 or even 4T5).

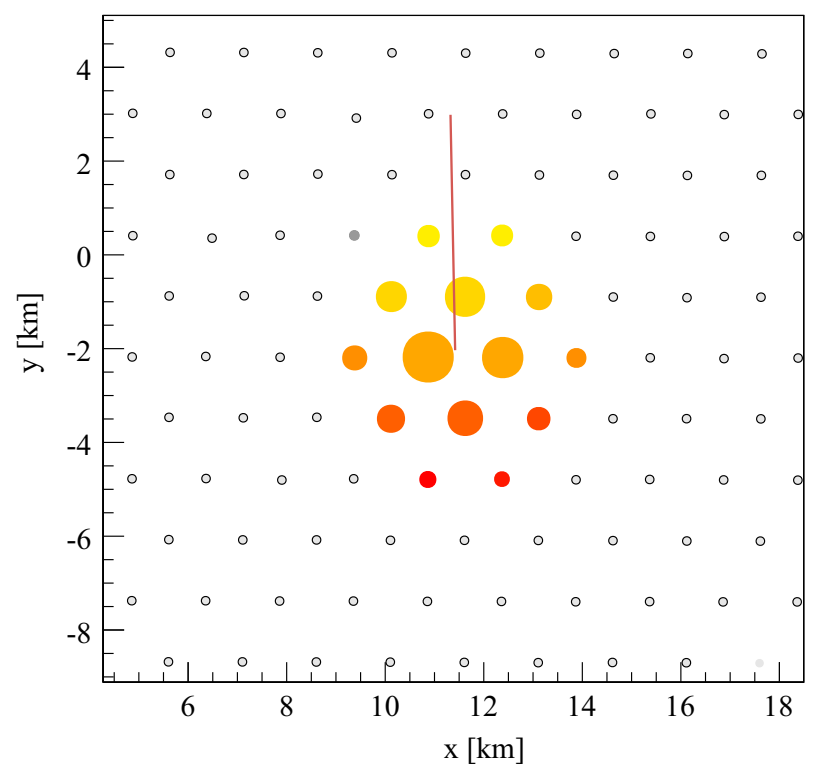

Fig. 37. An example of the sizes of signals which an air shower induces in the stations of the surface detector array. Note that the spacing of the regular grid is $1.5 \mathrm{~km}$. Colors represent the arrival time of the shower front from early (yellow [light shading]) to late (red [dark shading]) and the size of the markers is proportional to the logarithm of the signal. The line represents the shower arrival direction.

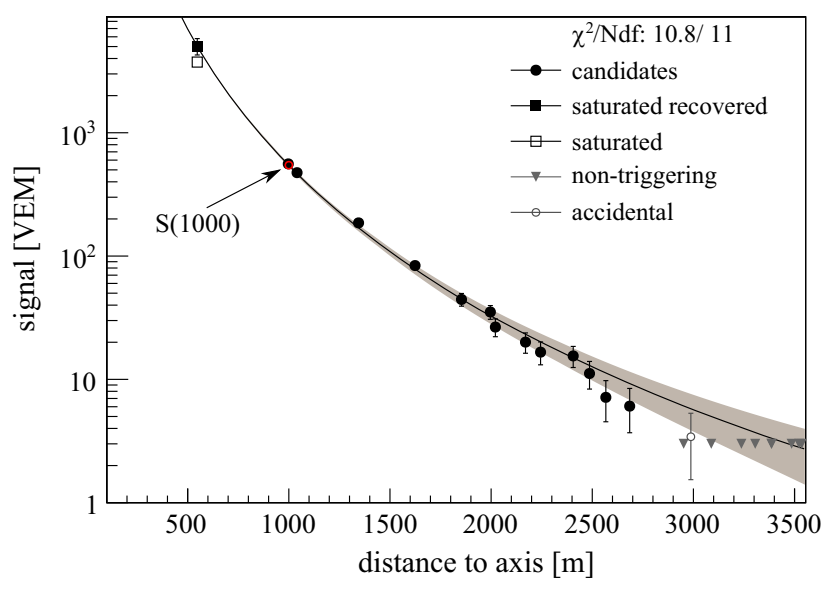

Fig. 38. Dependence of the signal size on distance from the shower core.

\subsection{Shower geometry}

A rough approximation for the arrival direction of the shower is obtained by fitting the start times of the signals, $t_{i}$, in individual SD stations to a plane front. For events with enough triggered stations, these times are described by a more detailed concentric-spherical model, see Fig. 35, which approximates the evolution of the shower front with a speed-of-light inflating sphere:

$c\left(t_{i}-t_{0}\right)=\left|\vec{x}_{\mathrm{sh}}-\vec{x}_{i}\right|$

where $\vec{x}_{i}$ are positions of the stations on the ground and where $\vec{x}_{\text {sh }}$ and $t_{0}$ are a virtual origin and a start-time of the shower development (see Fig. 36). From this 4-parameter fit the radius of curvature of the inflating sphere is determined from the time at which the core of the shower is inferred to hit the ground. 


\subsection{Lateral distribution function}

The impact points of the air showers on the ground, $\vec{x}_{\mathrm{gr}}$, are obtained from fits of the signals in SD stations. This fit of the lateral distribution function (LDF) is based on a maximum likelihood method which also takes into account the probabilities for the stations that did not trigger and the stations close to the shower axis with saturated signal traces. The saturation is caused by the overflow of the FADC read-out electronics with finite dynamic range and a modification of the signal due to the transition of the PMTs from a linear to a non-linear behavior. In the majority of cases the missing part of the signals are recovered using the procedure described in [116].

An example of the footprint on the array of an event produced by a cosmic ray with an energy of $(104 \pm 11) \mathrm{EeV}$ and a zenith angle of $(25.1 \pm 0.1)^{\circ}$ is shown in Fig. 37. The lateral distribution of the signals is depicted in Fig. 38. The function employed to describe the lateral distribution of the signals on the ground is a modified Nishimura-Kamata-Greisen function [117,118]:

$S(r)=S\left(r_{\mathrm{opt}}\right)\left(\frac{r}{r_{\mathrm{opt}}}\right)^{\beta}\left(\frac{r+r_{1}}{r_{\mathrm{opt}}+r_{1}}\right)^{\beta+\gamma}$

where $r_{\mathrm{opt}}$ is the optimum distance, $r_{1}=700 \mathrm{~m}$ and $S\left(r_{\mathrm{opt}}\right)$ is an estimator of the shower size used in an energy assignment. For the SD array with station spacing of $1.5 \mathrm{~km}$ the optimum distance [119] is $r_{\text {opt }}=1000 \mathrm{~m}$ and the shower size is thus $S(1000)$. The parameter $\beta$ depends on the zenith angle and shower size. Events up to zenith angle $60^{\circ}$ are observed at an earlier shower age than more inclined ones, thus having a steeper LDF due to the different contributions from the muonic and the electromagnetic components at the ground. For events with only 3 stations, the reconstruction of the air showers can be obtained only by fixing the two parameters, $\beta$ and $\gamma$ to a parametrization obtained using events with a number of stations larger than 4 .

The reconstruction accuracy of $S(1000), \sigma_{S(1000)}$, is composed of three contributions: a statistical uncertainty due to the finite number of particles producing a signal in a given SD station and the limited dynamic range of the signal detection; a systematic uncertainty due to assumptions on the shape of the lateral distribution function; and an uncertainty due to shower-toshower fluctuations [120]. The last term contributes a factor of about $10 \%$, while the contribution of the first two terms depends on energy and varies from 20\% (at low energies) to 6\% (at the highest energies).

\subsection{Shower arrival direction}

Shower axis $\hat{a}$ is obtained from the virtual shower origin (of the geometrical reconstruction) and the shower impact point on the ground (from the LDF reconstruction):

$\hat{a}=\frac{\vec{x}_{\mathrm{sh}}-\vec{x}_{\mathrm{gr}}}{\left|\vec{x}_{\mathrm{sh}}-\vec{x}_{\mathrm{gr}}\right|}$.

To estimate an angular resolution of the whole reconstruction procedure a single station time variance is modeled [121] to take into account the size of the total signal and the time evolution of the signal trace. As shown in Fig. 39, the angular resolution achieved for events with more than three stations is better than $1.6^{\circ}$, and better than $0.9^{\circ}$ for events with more than six stations [40].

\subsection{Energy calibration}

For a given energy, the value of $S(1000)$ decreases with the zenith angle $\theta$ due to the attenuation of the shower particles and geometrical effects. Assuming an isotropic flux of primary cosmic rays at the top of the atmosphere, we extract the shape of the attenuation curve (see Fig. 40) from the data using the Constant Intensity Cut (CIC) method [39]. The attenuation curve $f_{\mathrm{CIC}}(\theta)$ has been fitted with a third degree polynomial in $x=\cos ^{2} \theta-\cos ^{2} \bar{\theta}$, i.e., $f_{\mathrm{CIC}}(\theta)=1+a x+b x^{2}+c x^{3}$, where $a=0.980 \pm 0.004, \quad b=$ $-1.68 \pm 0.01$, and $c=-1.30 \pm 0.45$ [11]

The median angle, $\bar{\theta}=38^{\circ}$, is taken as a reference point to convert $S(1000)$ to $S_{38} \equiv S(1000) / f_{\text {CIC }}(\theta)$. $S_{38}$ may be regarded as the signal a particular shower with size $S(1000)$ would have produced had it arrived at $\theta=38^{\circ}$.

To estimate the energy of the primary particle producing the air-showers recorded with the SD, the advantage comes from the hybrid detection: the air-showers that have triggered independently the FD and SD are used for the cross-calibration. Highquality hybrid events, as defined below, with reconstructed zenith angles less than $60^{\circ}$ are used to relate the shower size from SD to the almost-calorimetric measurement of the shower energy from $\mathrm{FD}, E_{\mathrm{FD}}$.

These hybrid events must be such that the reconstruction of an energy estimator can be derived independently from both the SD and FD parts of the event $[122,115]$.

Only a subsample of events that passes strict quality and field of view cuts is used. For the FD part of the event, we require an accurate fit of the longitudinal profile to the Gaisser-Hillas

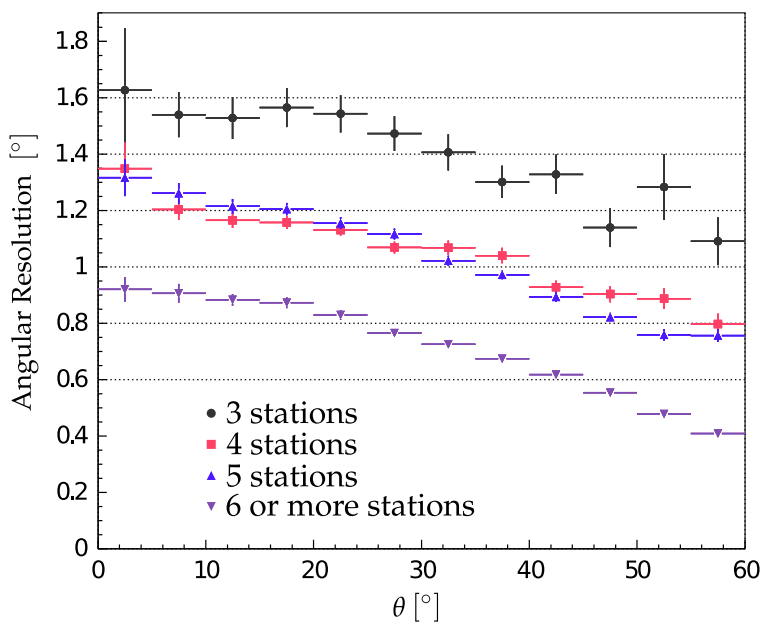

Fig. 39. Angular resolution as a function of the zenith angle $\theta$ for events with an energy above $3 \mathrm{EeV}$, and for various station multiplicities [40].

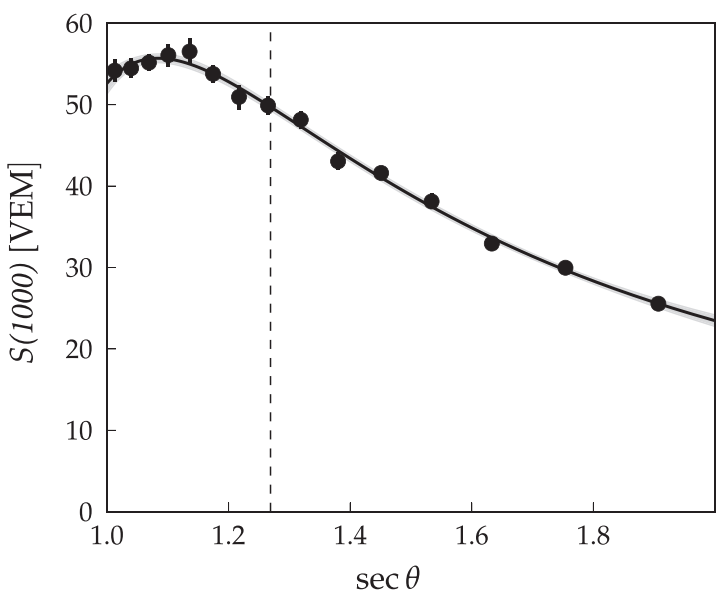

Fig. 40. Attenuation curve described by a third degree polynomial in $x=\cos ^{2} \theta-\cos ^{2} \bar{\theta}$ where $\bar{\theta}=38^{\circ}$ (denoted by the dashed vertical line). In this example the polynomial coefficients are deduced from $S(1000)$ dependence at $S_{38} \approx 50$ VEM which corresponds to an energy of about $10.5 \mathrm{EeV}$. 


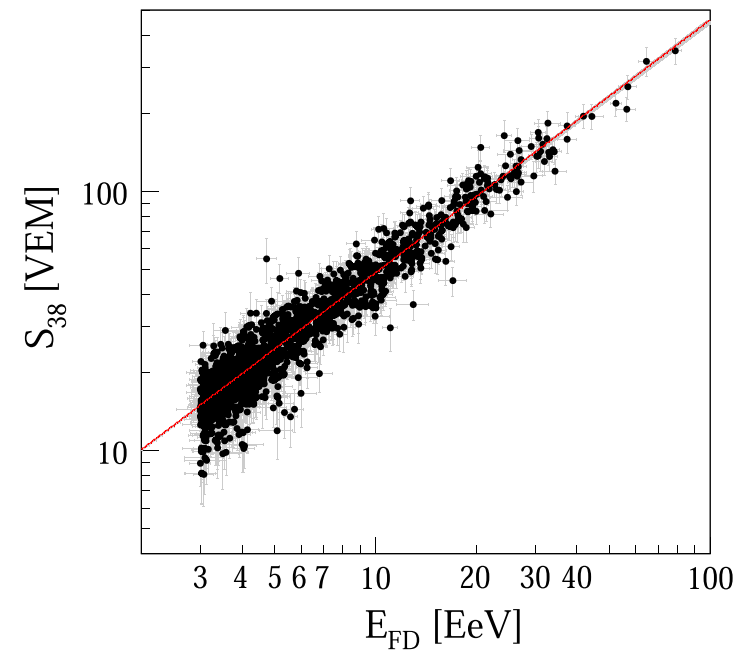

Fig. 41. Correlation between $S_{38}$ and $E_{F D}[11,122]$.

function. Furthermore, the depth of the shower maximum, $X_{\max }$, must be contained within the telescope field-of-view and measured with an accuracy better than $40 \mathrm{~g} / \mathrm{cm}^{2}$.

The uncertainty on the reconstructed $E_{\mathrm{FD}}$ is required to be less than $18 \%$. The final criteria for defining the calibration data sample include a selection of clear atmosphere conditions based on the measurements of the vertical aerosol optical depth, and on the cloud fraction measured by the lidar systems of the Observatory. To avoid any potential bias of the event selection on the mass of the primary particle, a fiducial cut on the slant depth range observed by the telescopes is also added, ensuring that the field of view is large enough to observe all plausible values of $X_{\max }$ for the geometry of each individual shower [122].

The final step in the calibration analysis leads to a relation between $S_{38}$ and $E_{\mathrm{FD}}$. The 1475 high quality hybrid events recorded between January 2004 and December 2012 which have an energy above the SD full efficiency trigger threshold [36] are used in the calibration. The correlation between the two variables is obtained from a maximum likelihood method [122,123] which takes into account the evolution of uncertainties with energy, as well as event migrations due to the finite energy resolution of the SD. The relation between $S_{38}$ and $E_{\mathrm{FD}}$ is well described by a single powerlaw function:

$E_{\mathrm{FD}}=A\left(S_{38} / \mathrm{VEM}\right)^{B}$

where the resulting parameters from the data fit are $A=(1.90 \pm 0.05) \times 10^{17} \mathrm{eV}$ and $B=1.025 \pm 0.007$ [11,124]. As can be seen in Fig. 41, the most energetic event used in this analysis has an energy of $79 \mathrm{EeV}$.

The resolution of the final SD energy estimator:

$E_{\mathrm{SD}}=A\left(S(1000) / f_{\mathrm{CIC}}(\theta) / \mathrm{VEM}\right)^{B}$

can be inferred from the distribution of the ratio $E_{\mathrm{SD}} / E_{\mathrm{FD}}$. Using the FD energy resolution of $7.6 \%$, the resulting SD energy resolution with its statistical uncertainty is $\sigma_{E_{S D}} / E_{\mathrm{SD}}=(16 \pm 1) \%$ at the lower energy edge in Fig. 41 and $(12 \pm 1) \%$ at the highest energies. Due to the large number of events accumulated until December 2012, the systematic uncertainty on the SD energy due to the calibration is better than $2 \%$ over the whole energy range. The systematic uncertainties in the energy scale, shown in Table 3, are dominated by the absolute FD calibration [124]. Further consistency checks are performed by joint calibration campaigns with the Telescope Array $[125,126]$.

The recorded dataset extends up to larger angles approaching $90^{\circ}$. For the inclined events, with zenith angles larger than $60^{\circ}$, we
Table 3

Systematic uncertainties in the energy scale.

\begin{tabular}{ll}
\hline Absolute fluorescence yield & $3.4 \%$ \\
Fluorescence spectrum and quenching parameters & $1.1 \%$ \\
Subtotal, fluorescence yield & $\mathbf{3 . 6} \%$ \\
Aerosol optical depth & $3-6 \%$ \\
Aerosol phase function & $1 \%$ \\
Wavelength dependence of aerosol scattering & $0.5 \%$ \\
Atmospheric density profile & $1 \%$ \\
Subtotal, atmosphere & $\mathbf{3 . 4 - 6 . 2} \%$ \\
Absolute FD calibration & $9 \%$ \\
Nightly relative calibration & $2 \%$ \\
Optical efficiency & $3.5 \%$ \\
Subtotal, FD calibration & $\mathbf{9 . 9} \%$ \\
Folding with point spread function & $5 \%$ \\
Multiple scattering model & $1 \%$ \\
Simulation bias & $2 \%$ \\
Constraints in the Gaisser-Hillas fit & $3.5-1 \%$ \\
Subtotal, FD profile reconstruction & $\mathbf{6 . 5 - 5 . 6} \%$ \\
Invisible energy & $\mathbf{3 - 1 . 5} \%$ \\
Statistical error of SD calibration fit & $\mathbf{0 . 7 - 1 . 8} \%$ \\
Stability of the energy scale & $\mathbf{5 \%}$ \\
Total & $\mathbf{1 4 \%}$ \\
\hline
\end{tabular}

Table 4

Key performance parameters for the Auger Observatory.

\begin{tabular}{ll}
\hline SD & \\
\hline SD annual exposure, $\theta<60^{\circ}$ & $\sim 5500 \mathrm{~km}^{2} \mathrm{sr}$ yr \\
T3 rate & $0.1 \mathrm{~Hz}$ \\
T5 events/yr, $E>3 \mathrm{EeV}$ & $\sim 14,500$ \\
T5 events/yr, $E>10 \mathrm{EeV}$ & $\sim 1500$ \\
Reconstruction accuracy $\left(S_{1000}\right)$ & $22 \%($ low $E)$ to $12 \%$ (high $E$ ) \\
Angular resolution & $1.6^{\circ}(3 \mathrm{stations})$ \\
& $0.9^{\circ}(>5 \mathrm{stations})$ \\
Energy resolution & $16 \%(\mathrm{low} E)$ to $12 \%$ (high $E$ ) \\
FD & \\
\hline On-time & \\
Rate per building & $\sim 15 \%$ \\
Rate per HEAT & $0.012 \mathrm{~Hz}$ \\
Hybrid & $0.026 \mathrm{~Hz}$ \\
\hline Core resolution & \\
Angular resolution & $50 \mathrm{~m}$ \\
Energy resolution (FD) & $0.6^{\circ}$ \\
$X_{\text {max }}$ resolution & $8 \%$ \\
\hline
\end{tabular}

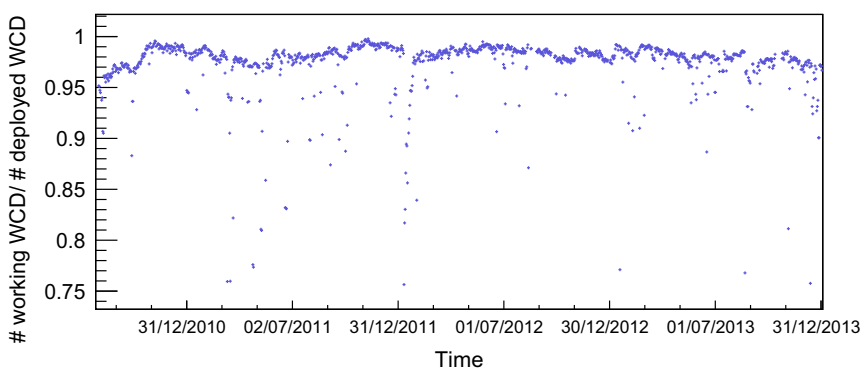

Fig. 42. Number of active SD stations normalized to the nominal number of SD stations in the array, as a function of time. (Note: $\mathrm{WCD}=$ water Cherenkov detector.)

employ a different reconstruction method. More details on the reconstruction of inclined events can be found in $[123,127,128]$. The energy range of full efficiency of the surface detector has been extended down to $3 \times 10^{17} \mathrm{eV}$ using the events recorded by the 


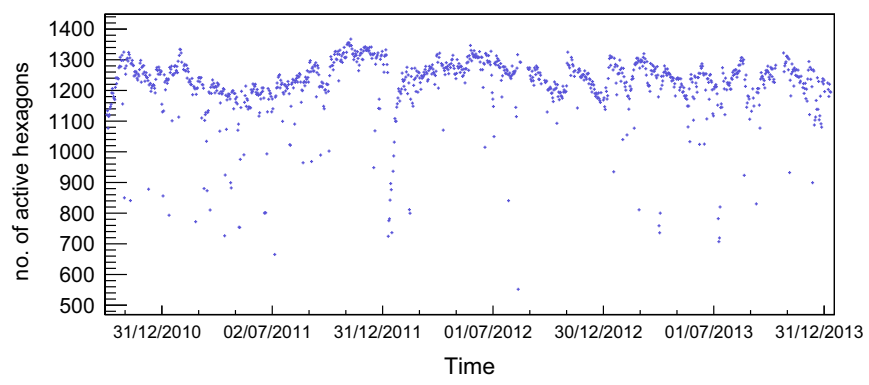

Fig. 43. Number of active hexagons as a function of time.

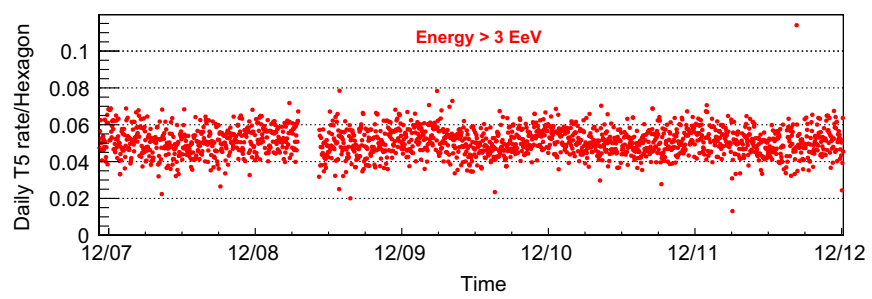

Fig. 44. Evolution of the daily T5 rate normalized to the number of hexagons for the period 2008-2012. The data gap in 2009 corresponds to a period during which the communications system experienced technical problems.

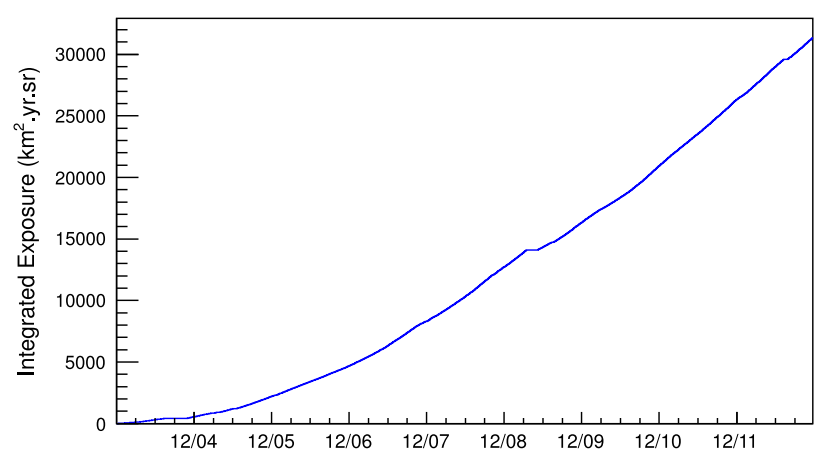

Fig. 45. Evolution of the exposure for vertical events $\left(\theta<60^{\circ}\right)$ between 1 January 2004 and 31 December 2012. The lack of exposure growth during an interval in 2009 corresponds to the data gap seen in Fig. 44.

$750 \mathrm{~m}$ array (see Section 13). The reconstruction of this subsample of events is described in [128-130].

\section{Performance characteristics of the Observatory}

\subsection{Key performance parameters}

In Table 4 are summarized some of the important parameters that characterize the performance of the Observatory. These parameters include the event rate of the detectors and the resolutions of the different reconstructed observables.

\subsection{Surface detector performance}

Stable data taking with the surface detector array started in January 2004 and the Observatory has been running in its full configuration since 2008. As described in Section 7, various parameters are continuously monitored to optimize the performance of the detectors and ensure reliable data.
The monitoring tool includes the so-called performance metrics to monitor the overall performance of the surface detector array. Relevant data useful for long term studies and for quality checks are stored in the Auger Monitoring database on a one-day basis. For example, mean values over one day of the number of active SD detectors and the number of active hexagons as well as the nominal value (expected value if all the detectors deployed were active) are available. As an example, Fig. 42 shows the number of active SD stations normalized to the nominal number of stations in the array for the last 4 years. This plot is a convolution of the status of the active stations and of the efficiency of the CDAS, which since the beginning has been better than $99.5 \%$.

Figure 43 shows the number of active hexagons for the same period. This variable is a key parameter since it is the basis of the exposure evaluation. Indeed, the offline T5 fiducial trigger, described in Section 11 selects only events for which the hottest station is surrounded by an active hexagon. Thus, above $3 \times 10^{18} \mathrm{eV}$, when the full efficiency of detection of the array is reached (at least three triggered tanks), the exposure is simply proportional to the integrated number of active hexagons during the period. The number of active hexagons fluctuates because of intermittent outages in electronics, communications, weather, and other factors [36]. Larger numbers of hexagons can be affected when the problems occur at the WLAN sector level. These outages can usually be resolved quickly.

The rate of events (T5 events) normalized to the average number of active hexagons is expected to be stable in time above the energy threshold of $3 \times 10^{18} \mathrm{eV}$, which can be seen in Fig. 44 .

Finally the integrated exposure between 1 January 2004 and 31 December 2012 is shown in Fig. 45. Since completion of the array in 2008 , the increase of the exposure has been about $5500 \mathrm{~km}^{2} \mathrm{sr}$ per year.

\subsection{Fluorescence detector performance}

The data taking of the FD can only take place under specific environmental conditions and is organized in night shifts. As described in Section 4.5, the telescopes are not operated when the weather conditions are unfavorable (high wind speed, rain, snow, etc.) and when the observed sky brightness (caused mainly by scattered moonlight) is too high. As a consequence, the shifters have to continuously monitor (see Section 7.4) the atmospheric and environmental conditions and judge the operation mode on the basis of the available information.

The performance of the fluorescence and hybrid data taking is then influenced by many effects. These can be external, e.g., lightning or storms, or internal to the data taking itself, e.g., DAQ failures. For the determination of the on-time of the Observatory in the hybrid detection mode it is, therefore, crucial to take into account all of these occurrences and derive a solid description of the data taking time sequence.

Data losses and inefficiencies can occur on different levels, from the smallest unit of the FD, i.e., one single photomultiplier (pixel) readout channel, up to the highest level, i.e., the combined SD/FD data taking of the Observatory.

The active time of the FD data acquisition is calculated using a minimum bias data stream with a less restrictive trigger condition. Since July 2007, the relevant information concerning the status of the FD detector has been read out from the Observatory monitoring system (see Section 7.4). An on-time dedicated database has been set up by storing the average variances and the on-time fraction of individual telescopes in time bins of $10 \mathrm{~min}$. The information on the veto due to the operation of the lidar or to an anomalous trigger rate on FD together with the status of the CDAS needed to form a hybrid event are also recorded. The 

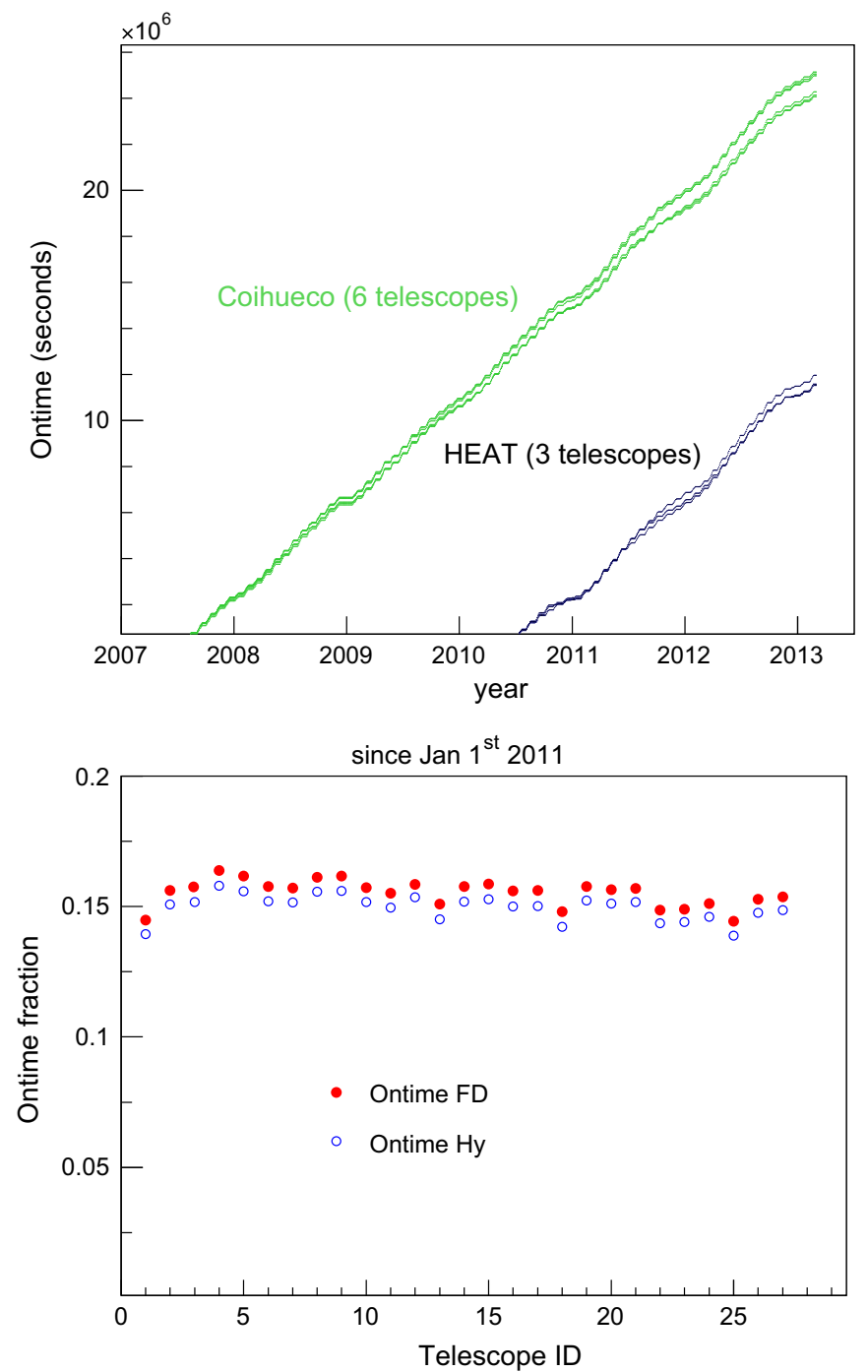

Fig. 46. Top: Accumulated on-time since 1 July 2007 for the 6 telescopes at Coihueco and for the 3 HEAT telescopes. Bottom: FD and hybrid on-time of individual telescopes since 1 January 2011. (1-6), (7-12), (13-18), (19-24), (2527) for the sites of Los Leones, Los Morados, Loma Amarilla, Coihueco and HEAT, respectively.

method to calculate the on-time of the hybrid detector is described in detail in Ref. [37].

The accumulated on-time is shown in Fig. 46, top, for the six telescopes at Coihueco and for the three HEAT telescopes. The average FD on-time (full circles) of individual telescopes since 1 January 2011 is shown in Fig. 46, bottom. Requiring that the CDAS is active defines the hybrid on-time (empty circles).

The time evolution of the full hybrid duty cycle over 9 years of operation is shown in Fig. 47, top, for all FD sites. Time bins are taken as the time intervals elapsed between two subsequent FD data taking shifts. The performance of the hybrid detector is compared to the nominal DAQ time (see Section 4.5) in the top panel of Fig. 47. In the bottom panel, the FD on-time is normalized to the time with high voltage $\mathrm{ON}$, leading to an average FD detector readiness of about $85 \%$ for all telescopes. The remaining inefficiency can be ascribed to different factors such as bad weather conditions (high wind load and/or rain) or high variances due to bright stars/planets crossing the field of view of the FD.

It should be noted that the FD site of Los Morados became operational in May 2005, Loma Amarilla starting from March 2007 and HEAT since September 2009. After the initial phase due to the

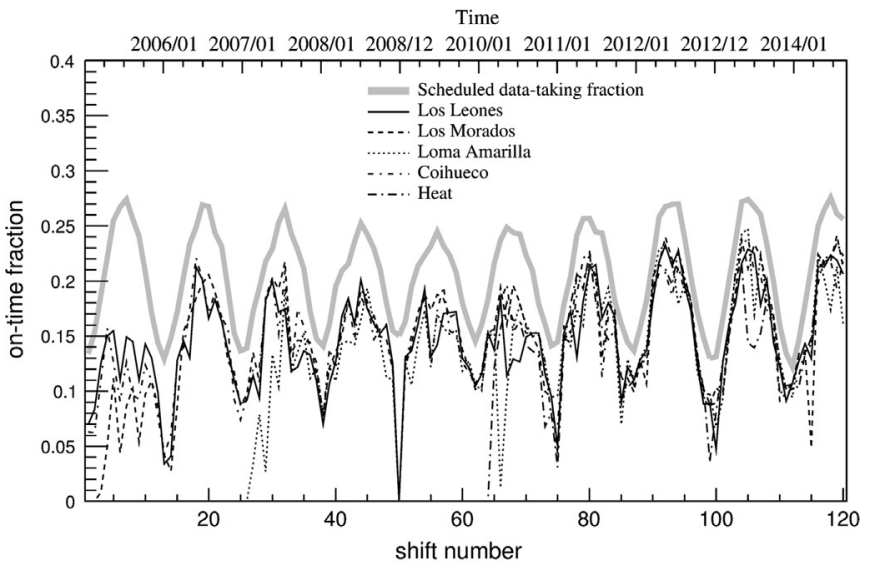

Time

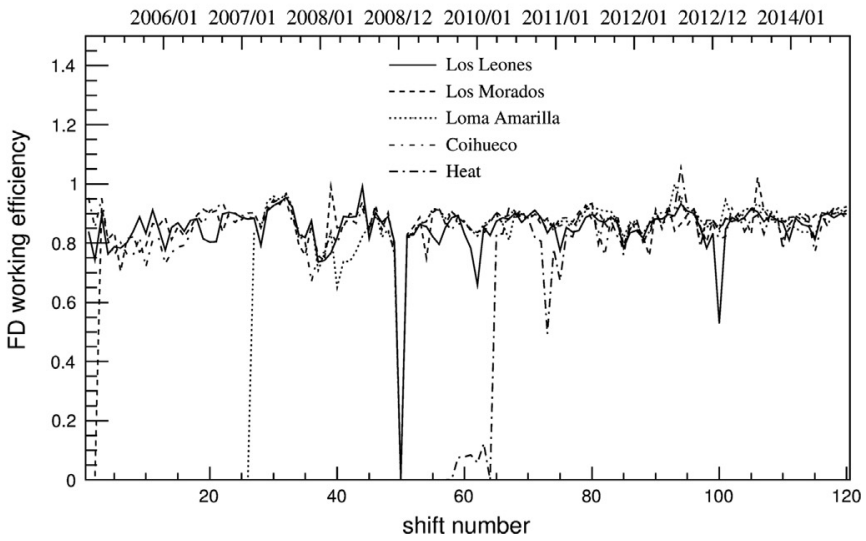

Fig. 47. Top: Time evolution of the average hybrid on-time fraction over nine years of operation of the Observatory. The thick gray line defines the scheduled data taking time fraction defined as the time periods with moon fraction lower than $70 \%$ and with the moon being below the horizon for more than $3 \mathrm{~h}$ (see also Section 4.5 for details). Bottom: readiness of the FD detector (see text for details).

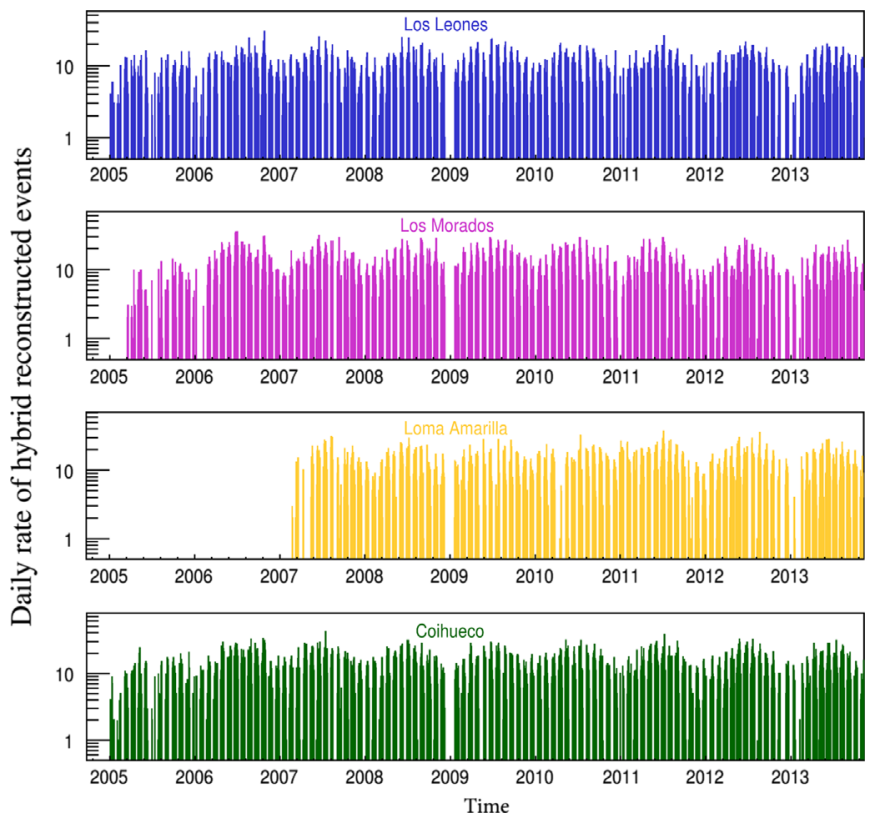

Fig. 48. Daily rate of hybrid reconstructed events as a function of year, starting in 2005, for (from top to bottom) Los Leones, Los Morados, Loma Amarilla and Coihueco, respectively.

start up of the running operations, the mean on-time is about $15 \%$ for all of the FD sites. Additionally, a seasonal modulation is visible, 

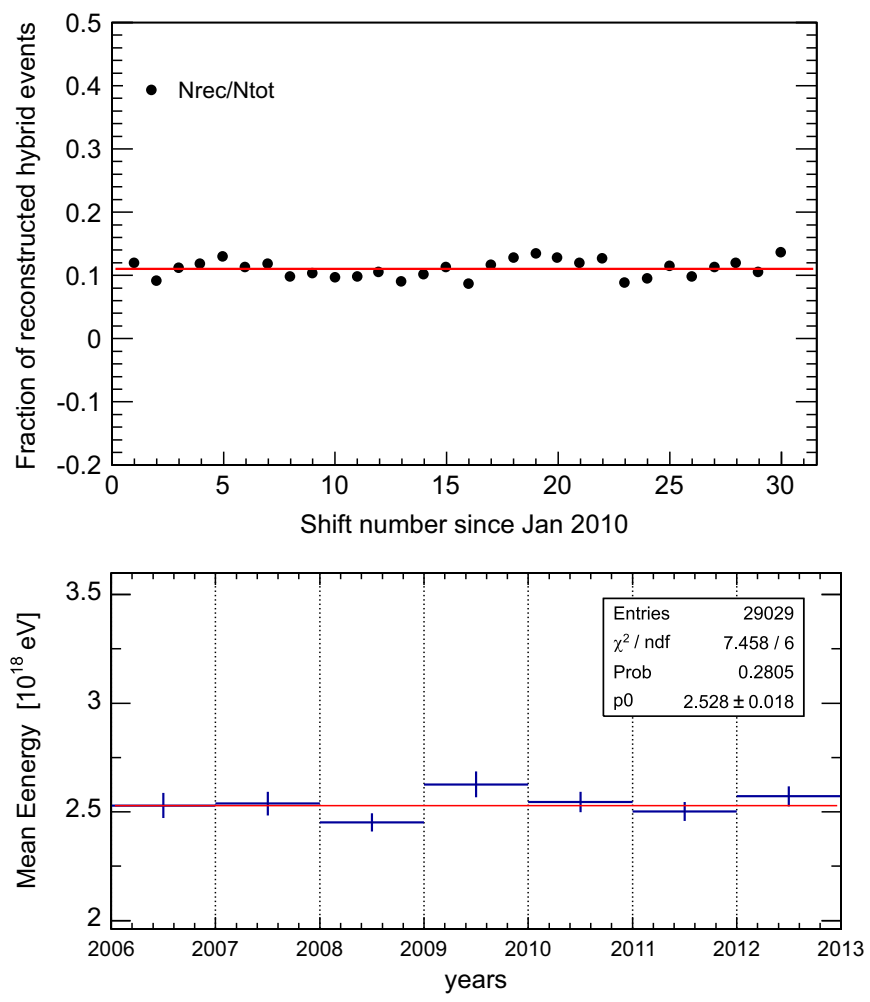

Fig. 49. Top: Fraction of all events that are well-reconstructed hybrids since 2010 . Bottom: Mean energy for reconstructed hybrid events.

since higher on-time fractions are observed in the austral winter during which the nights are longer.

\subsection{Time stability of the hybrid detector response}

The performance of the hybrid detector is demonstrated as a function of time using a sample of events fulfilling basic reconstruction requirements, such as a reliable geometrical reconstruction and accurate longitudinal profile and energy measurement. The daily rate of well-reconstructed hybrid events observed by individual FD sites is shown in Fig. 48 as a function of time, starting in 2005.

An important benchmark for the time stability of the hybrid detector response is the study of the effective on-time, defined as the fraction of all events that are well-reconstructed hybrids. Its time evolution, shown in Fig. 49 (top), exhibits quite a stable behavior over time. Moreover the mean energy of the hybrid events above $10^{18} \mathrm{eV}$, with distance to the shower maximum between 7 and $25 \mathrm{~km}$ (corresponding to $90 \%$ of the entire hybrid data sample), is shown as a function of time in Fig. 49 (bottom). All these features demonstrate the quality of the collected hybrid data and directly assess their long term stability.

\section{Enhancements to the Auger Observatory}

With the simultaneous and successful operation of the SD and FD, the Pierre Auger Collaboration has demonstrated the power of hybrid measurements. Since completion of the baseline construction, new enhancements were proposed to further extend the science reach of the Observatory.

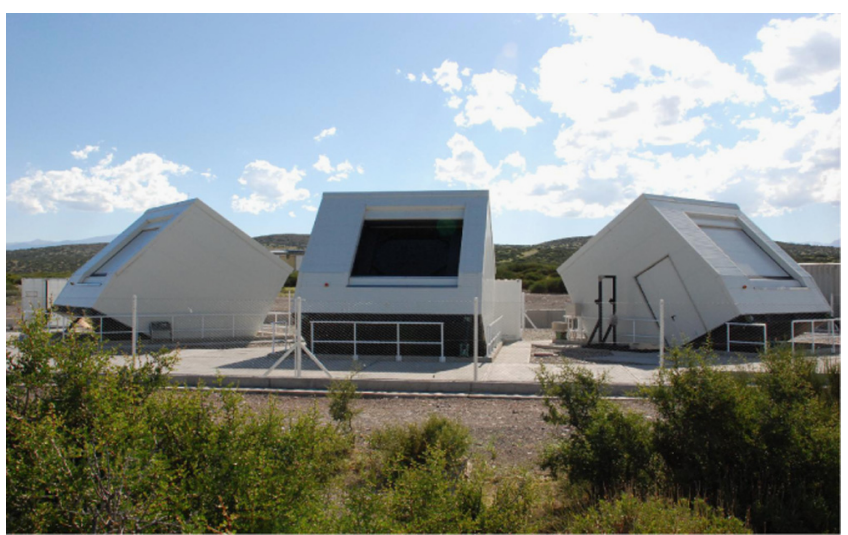

Fig. 50. Photo of the three HEAT telescopes in tilted mode. The container for DAQ slow control, and calibration hardware is behind the enclosure of the second telescope.

\subsection{High Elevation Auger Telescopes (HEAT)}

Three additional fluorescence telescopes with an elevated field of view were built about $180 \mathrm{~m}$ in front of the FD site at Coihueco [131]. These telescopes are very similar to the original fluorescence telescopes but can be tilted by $29^{\circ}$ upward with an electrically driven hydraulic system. These three telescopes work independently of other FD sites and form the "fifth site" of the Observatory. The HEAT telescopes were designed to cover the elevation range from $30^{\circ}$ to $58^{\circ}$, which lies above the field of view of the other FD telescopes. The HEAT telescopes allow a determination of the cosmic ray spectrum and $X_{\max }$ distributions in the energy range from below the second knee up to the ankle. The HEAT telescopes are depicted in Figs. 50 and 51.

The main objective of this extension was to lower the energy threshold of hybrid data to enable an unbiased detection of nearby low-energy showers. In combination with the SD, information from an infilled array of water Cherenkov detectors on a $750 \mathrm{~m}$ grid (see Section 13.2) close to the HEAT site, the energy range of high quality hybrid air shower measurements has been extended down to $10^{17} \mathrm{eV}$. In addition, close inclined shower events are detectable without any mass dependent bias or cuts up to the highest energies.

The layout of HEAT consists of three telescope enclosures and one container for DAQ slow control, and calibration hardware (see Fig. 50). The telescope enclosure consists of three main building blocks. First is the concrete foundation that supports the tilting mechanism and provides stability for the whole building. Second is the strong steel base plate, filled with concrete, on which all the sensitive optical elements are mounted. Finally a relatively lightweight steel container encloses the optical components and electronics. The base plate is connected to the foundation, while the steel enclosure is itself fixed to the plate. Similarly to the baseline telescopes, a shutter system is mounted on the steel enclosure, but of a different design.

The HEAT telescopes can be tilted using the hydraulic mechanism. The telescopes are parked in the horizontal position between the FD data taking periods to be accessible for maintenance, see Fig. 51. The same position is used for the absolute calibration of the HEAT telescopes and also for the cross-calibration with telescopes at Coihueco. All three HEAT telescopes are usually moved in the upward position before the first DAQ night and stay there during the whole data taking period.

To ensure sufficient mechanical stability during high winds and snow loads, all telescope components are connected to a heavy and stiff ground plate with adjustment mounting bolts. The mechanical stability is monitored by two types of sensors. The 
(a)

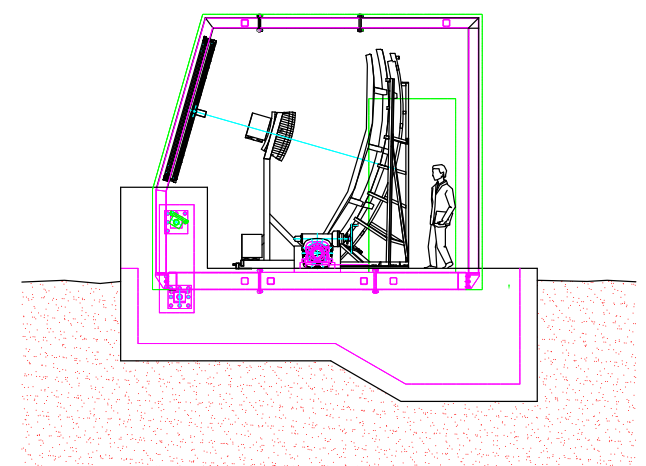

(b)

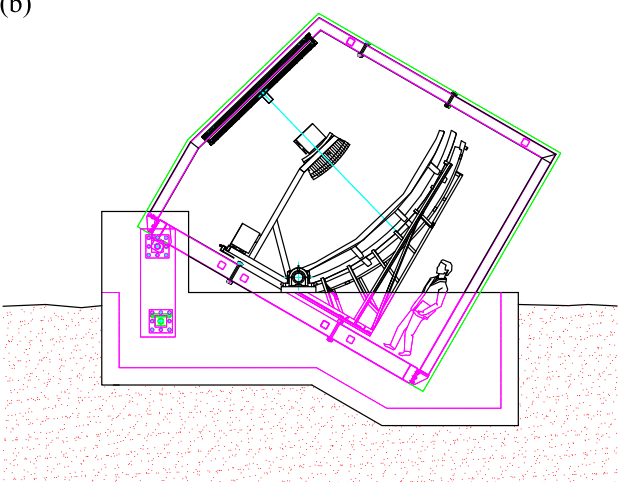

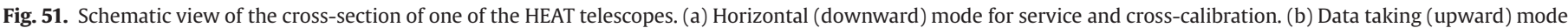
in tilted orientation.

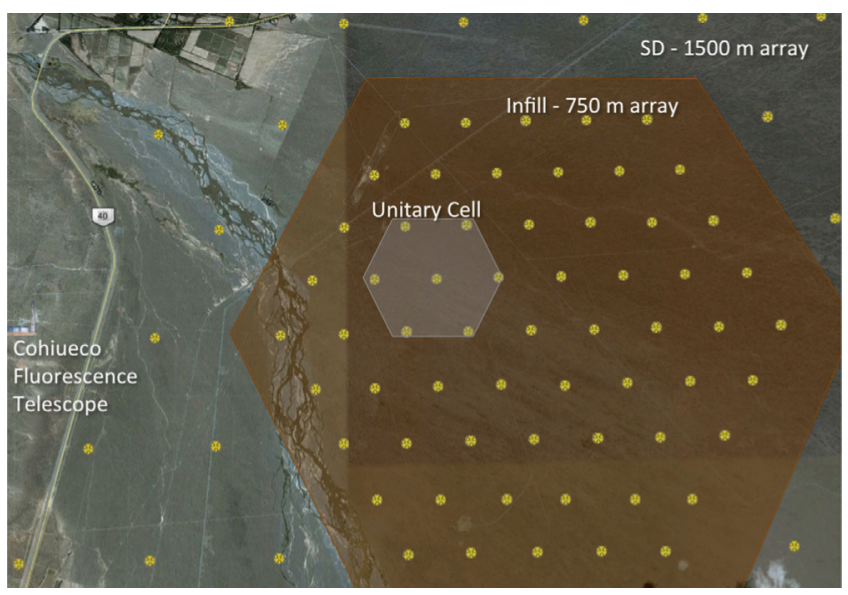

Fig. 52. AMIGA layout: an infill of surface stations with an inter-detector spacing of $750 \mathrm{~m}$ plus plastic scintillators of $30 \mathrm{~m}^{2}$ buried under $\approx 540 \mathrm{~g} / \mathrm{cm}^{2}$ of vertical mass to measure the muon component of the showers. The small shaded area indicates the prototype hexagon (Unitary Cell) of the muon detector which has been fully operational since March 2015. Two positions of UC are equipped with extra twin scintillators to allow the detector accuracy to be assessed while in the hexagon's center also $20 \mathrm{~m}^{2}$ were installed at $\approx 310 \mathrm{~g} / \mathrm{cm}^{2}$ to experimentally analyze the shielding power of the local soil.

first type is the inclination sensor that is used to measure the inclination variations at different points inside the HEAT shelters. The second type is needed to measure the distance variations between the optical components of the telescope. The distance sensors are not only used to measure the long term variations, but also the higher frequency variations that can take place in the telescope when subjected to strong winds or other similar conditions. The maximal allowed deformations and any movements after tilting lead to an angular offset less than $0.1^{\circ}$.

The response of the HEAT cameras was tested at multiple elevations using the relative calibration method (see Section 4.4.3). The effect on the signal of tilting HEAT is at the percent level or below, which matches the overall magnitude expected due to the direction of the Earth's magnetic field as seen by the PMTs. Also, the absolute calibration may be determined in the horizontal mode.

The Schmidt optics of the HEAT telescopes, camera body, PMTs, light collectors, etc., are the same as in the other sites. All three spherical mirrors are built up from hexagonal glass mirrors with vacuum-deposited reflective coatings.

A feature that sets HEAT apart from the classic Auger telescopes is its new electronics kit that can sample up to $40 \mathrm{MHz}$ instead of $10 \mathrm{MHz}$. In practice, a sampling rate of $20 \mathrm{MHz}$ (corresponding to a 50 ns FADC bin size) was chosen. The higher rate improves the measurement for close showers that have a correspondingly larger angular velocity - precisely the showers we are interested in observing with HEAT. From this it follows that the first level trigger interval was reduced to $50 \mathrm{~ns}$, whereas the second level trigger continues to operate every $100 \mathrm{~ns}$. The length (in time) of the FADC traces remains the same, so the number of bins doubles.

The trigger rate of the HEAT telescopes is high, particularly because of the Cherenkov light from low energy showers. Therefore the T4 trigger has been implemented to reduce the readout of the SD array for these low energy showers.

\subsection{Auger Muon and Infilled Ground Array (AMIGA)}

A dedicated detector to directly measure the muon content of air showers is being built. The AMIGA enhancement [132-134] is a joint system of water Cherenkov and buried scintillator detectors that spans an area of $23.5 \mathrm{~km}^{2}$ in a denser array with $750 \mathrm{~m}$ spacing nested within the $1500 \mathrm{~m}$ array (see Fig. 52). The area is centered $6 \mathrm{~km}$ away from the Coihueco fluorescence site. The $750 \mathrm{~m}$ array is fully efficient from $3 \times 10^{17} \mathrm{eV}$ onwards for air showers with zenith angle $\leq 55^{\circ}$ [129]. Although the infilled area is much smaller than the regular SD, the flux of cosmic rays increases steeply with decreasing energy such that this area is sufficient to observe a significant number of events and to study the region between the second knee [135] and the ankle of the cosmic ray spectrum.

The SD $750 \mathrm{~m}$ array was completed in September 2011 while the first prototype hexagon of buried scintillators, the Unitary Cell, has been fully operational since March 2015. This engineering array consists of seven water Cherenkov detectors paired with $30 \mathrm{~m}^{2}$ scintillators segmented in two modules of $10 \mathrm{~m}^{2}$ plus two of $5 \mathrm{~m}^{2}$ in each position. In addition, two positions of the hexagon were equipped with twin detectors (extra $30 \mathrm{~m}^{2}$ scintillators) to allow the accuracy of the muon counting technique to be experimentally assessed [136] and one position has $20 \mathrm{~m}^{2}$ of extra scintillators buried at a shallower depth to analyze the shielding features. In total, $290 \mathrm{~m}^{2}$ fully equipped plastic scintillators are operative in the Unitary Cell. The proven tools and methods used for the analysis of the $1500 \mathrm{~m}$ SD array data have been extended to reconstruct the lower energy events. The angular resolution for $E \geq 4 \times 10^{17} \mathrm{eV}$ is better than $1^{\circ}$ and the energy reconstruction is based on the lateral density of shower particles at the optimal distance of $450 \mathrm{~m}$ from the core [130].

The buried scintillators are the core of the detection system for the muonic component of air showers (the muon detector, MD). To effectively shield the electromagnetic component, the MD is placed under $\approx 540 \mathrm{~g} / \mathrm{cm}^{2}$ of vertical mass corresponding to a depth of $2.3 \mathrm{~m}$ in the local soil ( $\approx 20$ radiation lengths) while the shallower extra scintillators are at $\approx 310 \mathrm{~g} / \mathrm{cm}^{2}(1.3 \mathrm{~m})$. These 


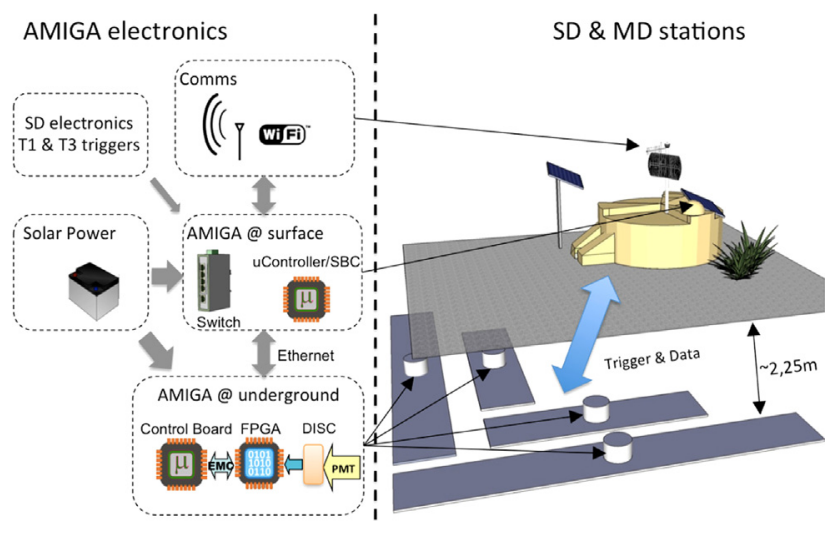

Fig. 53. AMIGA station: $\mathrm{SD}+\mathrm{MD}$ paired detectors. During the MD prototype phase the $30 \mathrm{~m}^{2}$ buried scintillators are segmented into 4 modules, $2 \times 10 \mathrm{~m}^{2}+2 \times 5 \mathrm{~m}^{2}$. To avoid shadowing effects by the water Cherenkov detector, there are $5 \mathrm{~m}$ of sideways clearance. The buried front end electronics is serviceable by means of an access pipe which is filled with local soil bags. Data are sent by a dedicated WiFi antenna.

shieldings impose a cutoff for vertical muons of around $1 \mathrm{GeV}$ and $0.6 \mathrm{GeV}$ respectively. The layout of $\mathrm{SD}+\mathrm{MD}$ paired stations is shown in Fig. 53. The scintillator surface of each MD station is highly segmented. It consists of modules made of 64 strips each. Strips are $4.1 \mathrm{~cm}$ wide $\times 1.0 \mathrm{~cm}$ thick and $400 \mathrm{~cm}$ or $200 \mathrm{~cm}$ long for the $10 \mathrm{~m}^{2}$ and $5 \mathrm{~m}^{2}$ modules, respectively. They consist of extruded Dow Styron 663W polystyrene doped by weight with $1 \%$ PPO (2,5-diphenyloxazole) and 0.03\% POPOP (1,4-bis(5-phenyloxazole-2-yl)benzene). They are completely wrapped with a thin white reflective layer of titanium dioxide $\left(\mathrm{TiO}_{2}\right)$ except for a central groove into which a wavelength shifting (WLS) optical fiber is installed. The light output uniformity is $\pm 5 \%$. Because the scintillators have an attenuation length of $\sim(55 \pm 5) \mathrm{mm}$, light is transported to a photomultiplier tube using the WLS fiber. The manifold of fibers of each module ends in an optical connector matched to a 64 multi-anode PMT from the Hamamatsu H8804 series. Scintillator strips are grouped in two sets of 32 strips on each side of the PMT and front end electronics board (see Fig. 54).

The bandwidth of the front end electronics is set to $180 \mathrm{MHz}$ to determine the pulse width. Signal sampling is performed by a Field Programmable Gate Array (FPGA) from the ALTERA Cyclone III series at $320 \mathrm{MHz}$. MD scintillator modules receive the trigger signal from their associated SD station. The lowest level trigger (T1) of the surface detectors is used. Once a T1 condition is fulfilled on the surface, its MD companion freezes a $6.4 \mu$ s data sample into a local buffer $-1.6 \mu$ s before and $4.8 \mu$ s after the T1. Data are then moved to an external RAM capable of storing 1024 triggers [137].

Incoming analog signals from each pixel of the PMT are digitized with a discriminator that provides the input to the FPGA. Samples can be either a logical " 1 " or " 0 " depending on whether the incoming signal was above or below a given (programmable) discrimination threshold. This method of one-bit resolution is very robust for counting muons in a highly segmented detector. This avoids missing muons due to simultaneous particle arrivals [138]. It relies neither on deconvolving the number of muons from an integrated signal, nor on the PMT gain or its fluctuations, nor on the point of impact of the muon and the corresponding light attenuation along the fiber. It also does not require a thick scintillator to control Poissonian fluctuations in the number of single photoelectron pulses per impinging muon [139]. The MD station power is supplied by an additional solar panel and battery box (see Fig. 53).

Whereas the data of the $750 \mathrm{~m}$ array are transmitted over the same SD radio as for the regular array, a dedicated telecommunication system based on WiFi 802.11g standard is used for MD data transmission during the prototype phase. The system is provided

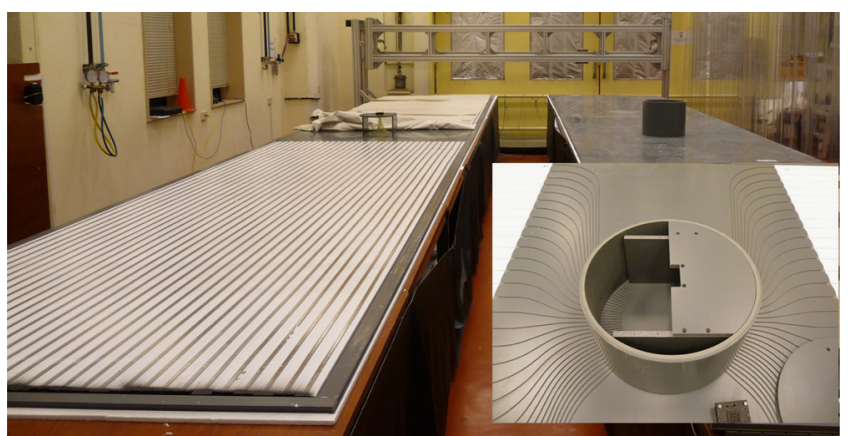

Fig. 54. AMIGA scintillator detector, illustrating the assembly of a $10 \mathrm{~m}^{2}$ module. Strips are grouped in two sets of 32 strips on each side of the electronics dome located at the center of the detector. The inset shows a detailed view of the manifold fiber routing and optical connector. The multi-anode PMT and front end electronics board are hosted in the central dome. Once deployed, access to the buried setup is provided by an inspection pipe.

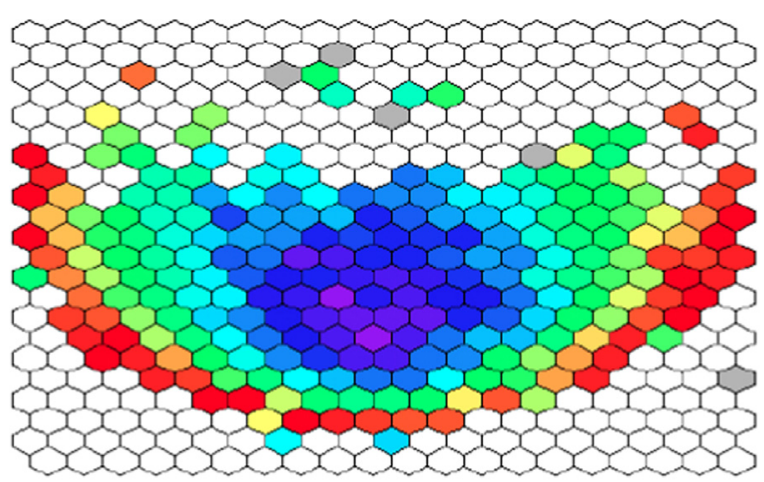

Fig. 55. A typical ELVES candidate event as seen in the FD. (For interpretation of the references to color in this figure caption, the reader is referred to the web version of this paper.)

by an extra antenna located on the SD mast as indicated in Fig. 53. MD data are sent to the CDAS only at T3 level. As WiFi based telecommunication has proven to satisfy the network throughput and data transfer requirements for SD T2s and SD + MD T3s data, it is foreseen that this system will be used for the whole AMIGA detector.

\section{Other capabilities of the Observatory}

\subsection{Space weather}

The rate of background low energy particles detected by the water Cherenkov detectors of the Observatory is recorded every second and transmitted to CDAS. This rate, of around 2000 particles/s per detector, is used to monitor the stability of operations. The particles themselves are residual components of air showers initiated by primary cosmic rays with a mean energy of about $90 \mathrm{GeV}$. We have observed that this rate correlates strongly with neutron monitors measuring Forbush decreases [140]. Measuring the flux of secondaries with great accuracy allows the Observatory to contribute to the "Space Weather" program [141]. These data are available on the Public Event Display of the Observatory web site [142].

\subsection{ELVES}

ELVES (an acronym for Emissions of Light and Very low frequency perturbations due to Electromagnetic pulse Sources) 
are transient luminous events produced by heating, ionization, and subsequent optical emission due to intense electromagnetic pulses radiated by both positive and negative lightning discharges. These intense flashes of light appear in the night sky as rapidly expanding quasi-circular fronts; generated at 80-95 km altitudes, they are visible at distances of several hundred kilometers. The original pulse lasts less than $20 \mu \mathrm{s}$, but the propagating light front is visible for a few ms [143]. The first clear observation of ELVES was made using a high-speed photometer [144]; more recently such phenomena were studied using both ground based photometers [145-147] and satellite missions [148]. After the first serendipitous observation of an ELVES event during a FD shift [99] further studies, done on a pre-scaled sample of data taken in the period 2008-2011, have shown that the FD is ideally suited for detailed studies of ELVES. A new, modified third level trigger algorithm was implemented in March 2013 [149] to increase the detection and recording efficiency of these events. Since then, a large fraction of these events is regularly recorded by two or three FD eyes allowing a stereo reconstruction of the light emission. Also, since January 2014, when an ELVES trigger is received, the FD camera readout is extended to $300 \mu$ s to allow observation of the light emitted vertically above the causative lightning.

Auger detected 305 events in nine months of running in 2013, and 581 events (including 127 stereo and 20 triplets) in all of 2014. The distance of the causative lightning ranged from 300 to $1000 \mathrm{~km}$, as determined by comparison with WWLLN data [150]. More than $40 \%$ of these events are correlated to lightning detected by WWLLN.

Figure 55 shows the light propagation pattern in an ELVES candidate event: the earliest triggered pixels are in blue (near the center of the image), while the latest are in red (near the periphery of the image).

\section{Outreach}

The scale and scope of the physics explored at the Pierre Auger Observatory offer significant opportunities for outreach both to the local community and beyond to the collaborating countries. Education, outreach and public relations have been an integral part of the Auger organization from the beginning when these activities were included as a level two management task group. The goals of the Outreach and Education Task are to encourage and support a wide range of efforts that link Auger scientists and the science of particle astrophysics, particle physics and related technologies to the public and especially to schools. Outreach focused on the communities surrounding the Observatory has fostered a remarkable amount of goodwill, which has contributed significantly to the success of the project.

The Auger Collaboration initiated outreach first locally as a way to become better integrated into the community during the construction phase of the Observatory. Later outreach activities spread to the participating institutions but on a worldwide scale, including the Internet.

The heart of local outreach activities is the Auger Visitor Center (VC), located in the central office and data acquisition building on the Observatory campus. A staff member dedicated to outreach gives presentations and tours to visitors that are mostly from the area but often from all over Argentina and even from 25 other countries worldwide. Many of the visitors are in the area because of the proximity of the Las Leñas ski area and other area tourist attractions. Over 90,000 people have attended the lectures in the Visitor Center since it opened in 2001. The impact of these visits can be seen from the still increasing interest and the comments in the guest book. The VC, which seats up to 50 people, is outfitted with multimedia equipment and contains a number of displays illustrating features of the Observatory. These displays include a full size SD station, a quarter sized model of an FD mirror, a spark chamber, a Geiger counter, a number of posters that explain the science and detectors of the Observatory and a library of books in several languages.

Many schools outside of Malargüe, some over $100 \mathrm{~km}$ away, have trouble bringing their students to the Visitor Center. A rural schools education program, funded by donations from the collaboration, sponsors a dedicated team of Observatory staff members and collaborators, who give presentations on Auger and science generally. The visiting team not only goes to these schools to give information on Auger but also helps to enhance the learning environment by providing learning materials or helping with infrastructure improvements such as connection to the Internet. The effect of these visits on the students is enormous and creates an atmosphere of good will towards the Observatory.

Every two years a science fair is organized by the Observatory. The fair targets both elementary schools and high schools, and is still growing. The latest fair hosted 36 entries with schools from all over Mendoza Province participating. The exhibits and presentations of the participants were judged by international members of the Auger Collaboration. The interaction of the participants with the Auger scientists reinforces the connection between Auger and surrounding communities.

Each of the surface detector stations placed in the field was given a name. During deployment, the stations were often named after the daughter or son of the nearby puestero (farmer). One of the results has been that there has been a negligible amount of vandalism even though the detectors are spread over $3000 \mathrm{~km}^{2}$, with a number only a few meters from a road.

Every November a collaboration meeting is scheduled to overlap with the annual Malargüe Days celebration and a large group of collaborators march in the celebration parade behind a colorful Auger banner. These close contacts together with the local outreach activities have instilled a sense of ownership of the Observatory by the community.

Our dedication to education is also clear from the construction of the James Cronin School, a secondary school in Malargüe inaugurated in 2006. The school, built from donated funds, was named for one of the founders of the Pierre Auger Observatory for his contribution to the local community. Members of the collaboration have also been instrumental in helping to bring a new planetarium, one of the most modern in Argentina, to Malargüe.

For outreach on a larger scale the Collaboration provides cosmic ray event data on the public Auger Web site (www.auger. org) in nearly real time along with information, photos, videos and teaching materials. This material not only explains the mission of the Observatory, but also contains educational material on several aspects of the measurement of cosmic rays and the history of these measurements. Furthermore, it provides manuals on how to work with and analyze the Auger public data set. The online event display, coupled to the public data set, is a useful tool to provide insight to students in what is measured and how it is interpreted. These materials are mostly aimed at students at the high school level or above. An online analysis interface called VISPA [151] has been set up to allow students to work with and analyze these data.

Outreach has been an important part of the activities of the Auger Project. Our close relationship with the people of Malargüe and the other local communities as a result of our outreach activities has not only made our work comfortable and rewarding but has, indeed, contributed to the success of the Observatory. Among the collaborating institutions many innovative outreach ideas have sprung from our research. Because we can easily show their continuous presence around us, cosmic rays are an effective way to excite young people about the wonders and science of the cosmos. 


\section{Further developments}

Even as the Pierre Auger Observatory was under construction, new ideas for methods of air shower detection were being developed. These ideas became more focused as the underlying physics of cosmic rays was revealed by analysis of the expanding data set.

Research and development is currently underway on two detection techniques which could complement the array of water Cherenkov and air fluorescence detectors. The first is radio detection, a technique that was first pursued many years ago but is now benefitting from recent advances in electronics. The second depends on the possibility that microwave radiation, arising from molecular bremsstrahlung of electromagnetic shower particles in air, is isotropic and strong enough to be effectively recorded. If successful, one or both of these techniques could substantially extend the power of cosmic ray air shower detectors.

\subsection{Radio research program}

High-energy cosmic ray air showers generate radio emission via two processes: one is a geomagnetic, current-induced emission mechanism [152]; the other is a charge-excess mechanism [153]. The observation of air showers with radio detection techniques can be done at all times (day and night). Moreover, radio signals are sensitive to the development of the electromagnetic component of particle showers in the atmosphere of the Earth and, in particular, to the depth of the shower maximum or mass of the incoming cosmic ray [154]. Radio detection of air showers started in the 1960s, and the achievements in those days have been presented in reviews by Allan [155] and Fegan [156]. More recent developments are based on initial studies performed by the LOPES [157] and the CODALEMA [158] collaborations and the LOFAR radio telescope [159]. In the last 10 years the radio detection technique in the $\mathrm{MHz}$ region has been revived and the present radio detector arrays for cosmic ray research are equipped with low noise and high rate analog-to-digital converters. Simultaneously, the number of stations within these arrays has grown from less than ten to more than one thousand. The questions to be addressed in the VHF band (30$300 \mathrm{MHz}$ ) are: can we use radio signals to determine the primary energy, the arrival direction, and the mass of cosmic rays with accuracies which are equal to or better than those obtained by other techniques? If yes, can we build a large surface detector array based on the radio detection technique for an affordable price?

The Pierre Auger Collaboration has started a research program to answer both questions through a stepwise approach. Since 2009 the activities are coordinated within the Auger Engineering Radio Array (AERA), which is based on work within the Collaboration using various prototypes at the site of the Pierre Auger Observatory [160-162]. As a first step, the emission mechanisms need to be understood. The contributions of the main emission mechanisms have been measured recently $[163,164]$. The fraction of the charge-excess emission relative to the geomagnetic emission varies from $\sim 5 \%$ for very inclined showers at $50 \mathrm{~m}$ from the shower axis to more than $20 \%$ for vertical showers at $250 \mathrm{~m}$ from the shower axis. As a second step the data obtained with radio detection stations deployed at the Observatory will be used to check their sensitivity with respect to the determination of the air shower parameters. We take advantage of the existing infrastructure of the Observatory: its surface detector, its fluorescence detector and its low energy enhancements HEAT and AMIGA $[131,133]$. To help answer the questions, hardware and software are being developed to study the required specifications and performance of solitary radio stations as a blueprint for a large array. Within the same research program, a rigorous effort has been and is being made to understand the emission processes using our current knowledge of the development of air showers. Simultaneously, experiment and theory are being connected through software tools where end-to-end simulations and data analysis can be performed within the same software package [69].

The scientific goals of the AERA project are as follows: (1) calibration of the radio emission from the air showers, including subdominant emission mechanisms; (2) demonstration at a significant scale of the physics capabilities of the radio technique, e.g., energy, angular, and mass resolutions; and (3) measurement of the cosmic ray composition from 0.3 to $5 \mathrm{EeV}$, with the goal of elucidating the transition from galactic to extragalactic cosmic rays.

Each radio detection station is comprised of a dual polarization antenna, sensing the electric field in the north/south and east/west directions, associated analog and digital readout electronics, an autonomous power system and a communication link to a central data acquisition system. The antennas are sensitive between 30 and $80 \mathrm{MHz}$, chosen as the relatively radio quiet region between the shortwave and FM bands. AERA deployment began in 2010 with 24 stations. These stations are equipped with logarithmic periodic dipole antennas and are connected via a fiber optic link to a central data acquisition site. Stable physics data taking started in March 2011, and the first hybrid detection of cosmic ray events by radio, fluorescence, and surface particle detectors was recorded in April 2011. In May 2013 an additional 100 stations were installed. They are equipped with butterfly antennas. Detailed simulations and measurements demonstrated that butterfly antennas perform better for narrow pulse detection as compared to the logarithmic periodic dipole antennas [165]. The additional stations are connected through a wireless communication system to a central data acquisition system. AERA successfully deployed 25 additional radio stations in March 2015. AERA now includes 153 radio detection stations, spread over an area of $17 \mathrm{~km}^{2}$. The detector spacings range from $150 \mathrm{~m}$ to $750 \mathrm{~m}$, which enables the full exploitation of radio detection of air showers as envisioned in the AERA technical design report.

\subsection{Microwave research program}

Recent results of a test beam experiment at SLAC [166] showed that it could be possible to use microwave radiation to detect extensive air showers. This radiation, expected to be isotropic and broad in frequency, is interpreted [166] as molecular bremsstrahlung (MBR) produced by the scattering of low energy electrons in the weakly ionized plasma produced by the shower with the neutral molecules of the atmosphere. The Auger collaboration is pursuing an active $R \& D$ program to determine if a detector sensitive to MBR would be a suitable alternative for the study of ultrahigh energy cosmic rays.

This R\&D program $[167,168]$ consists of three different setups installed at the Observatory. The AMBER and MIDAS experiments use radiotelescope style detectors intended for the observation of the shower longitudinal development in the same manner as an FD. In the EASIER setup on the other hand, SD tanks are instrumented with smaller radio receivers that take advantage of the enhancement of the signal when the shower is observed close to its axis.

Installation of the microwave detectors was finalized in September 2012. A previous result by the MIDAS detector [169], obtained in Chicago, places tight constraints on the amount of microwave signal emitted and its scaling with the energy of the shower [170]. The ongoing work to identify showers detected at the same time in the SD and in one of the microwave detectors already yielded the first unambiguous detection of a cosmic ray shower in the EASIER setup in June 2011 [168]. 


\section{Final remarks}

The Pierre Auger Observatory is the world's largest cosmic ray observatory. The Observatory is highly productive with very high efficiency data accumulation and sophisticated atmospheric monitoring for accurate interpretation of showers. There have been recent advances enabling the extraction of longitudinal development information from surface detector information alone. New analysis methods using both the fluorescence detectors and the surface array have improved the determination of primary composition. At the same time the recently completed HEAT and AMIGA infilled enhancements now extend the reach of the detector to cover the critical galactic/extragalactic transition region. The Observatory is also serving as a test bed for advanced detector technologies for the next generation of cosmic ray experiments.

Current source scenarios assume that particle acceleration takes place at sites distributed similarly to the matter distribution in the universe, with energy loss processes leading to the observed flux suppression (GZK effect) and arrival direction anisotropy. However, Auger data on shower development fluctuations, as well as other composition sensitive observables, require consideration of a rather different interpretation: that the observed flux suppression is indicating the upper limit of the power of the accelerator. It may be that the upper end of the cosmic ray energy spectrum is dominated by particles from a single source or source population, possibly within the GZK horizon, for which the upper limit of particle acceleration almost coincides with the energy of the GZK suppression. To answer this question, the Pierre Auger Collaboration is planning an upgrade to the Observatory to enable a determination of the primary composition on an event by event basis at the highest energies. The required electron-muon discrimination in the surface detector stations will be achieved by installing a $4 \mathrm{~m}^{2}$ scintillation detector on top of each water Cherenkov detector. Once approved, the upgrade is expected to be completed by 2018 with Observatory operation through 2025 .

\section{Acknowledgments}

The successful installation, commissioning, and operation of the Pierre Auger Observatory would not have been possible without the strong commitment and effort from the technical and administrative staff in Malargüe.

We are very grateful to the following agencies and organizations for financial support: Comisión Nacional de Energía Atómica, Fundación Antorchas, Gobierno De La Provincia de Mendoza, Municipalidad de Malargüe, NDM Holdings and Valle Las Leñas, in gratitude for their continuing cooperation over land access, Argentina; the Australian Research Council; Conselho Nacional de Desenvolvimento Científico e Tecnológico (CNPq), Financiadora de Estudos e Projetos (FINEP), Fundação de Amparo à Pesquisa do
Estado de Rio de Janeiro (FAPERJ), São Paulo Research Foundation (FAPESP) Grants \# 2010/07359-6 and \# 1999/05404-3, Ministério de Ciência e Tecnologia (MCT), Brazil; MSMT-CR LG13007, 7AMB14AR005, CZ.1.05/2.1.00/03.0058 and the Czech Science Foundation Grant 14-17501S, Czech Republic; Centre de Calcul IN2P3/CNRS, Centre National de la Recherche Scientifique (CNRS), Conseil Régional Ile-de-France, Département Physique Nucléaire et Corpusculaire (PNC-IN2P3/CNRS), Département Sciences de l'Univers (SDU-INSU/CNRS), Institut Lagrange de Paris, ILP LABEX ANR-10-LABX-63, within the Investissements d'Avenir ProgrammeANR-11-IDEX-0004-02, France; Bundesministerium für Bildung und Forschung (BMBF), Deutsche Forschungsgemeinschaft (DFG), Finanzministerium Baden-Württemberg, Helmholtz Alliance for Astroparticle Physics (HAP), Helmholtz-Gemeinschaft Deutscher Forschungszentren (HGF), Ministerium für Wissenschaft und Forschung, Nordrhein Westfalen, Ministerium für Wissenschaft, Forschung und Kunst, Baden-Württemberg, Germany; Istituto Nazionale di Astrofisica (INAF), Istituto Nazionale di Fisica Nucleare (INFN), Ministero dell'Istruzione, dell'Università e della Ricerca (MIUR), Gran Sasso Center for Astroparticle Physics (CFA), CETEMPS Center of Excellence, Italy; Consejo Nacional de Ciencia y Tecnología (CONACYT), Mexico; Ministerie van Onderwijs, Cultuur en Wetenschap, Nederlandse Organisatie voor Wetenschappelijk Onderzoek (NWO), Stichting voor Fundamenteel Onderzoek der Materie (FOM), Netherlands; National Centre for Research and Development, Grant nos. ERA-NETASPERA/01/11 and ERA-NET-ASPERA/02/11, National Science Centre, Grant nos. 2013/08/M/ST9/00322, and 2013/08/M/ST9/00728 and HARMONIA 5 - 2013/10/M/ST9/00062, Poland; Portuguese national funds and FEDER funds within COMPETE - Programa Operacional Factores de Competitividade through Fundação para a Ciencia e a Tecnologia, Portugal; Romanian Authority for Scientific Research ANCS, CNDI-UEFISCDI partnership projects nos. 20/2012 and nr.194/2012, project nos. 1/ASPERA2/2012 ERA-NET, PN-II-RU-PD-2011-3-0145-17, and PN-II-RU-PD-20113-0062, the Minister of National Education, Programme for research - Space Technology and Advanced Research - STAR, project number 83/2013, Romania; Slovenian Research Agency, Slovenia; Comunidad de Madrid, FEDER funds, Ministerio de Educación y Ciencia, Xunta de Galicia, European Community 7th Framework Program, Grant no. FP7-PEOPLE-2012-IEF-328826, Spain; Science and Technology Facilities Council, United Kingdom; Department of Energy, Contract no. DE-AC02-07CH11359, DE-FR02-04ER41300, DE-FG02-99ER41107 and DE-SC0011689, National Science Foundation, Grant no. 0450696, The Grainger Foundation, USA; NAFOSTED, Vietnam; Marie Curie-IRSES/EPLANET, European Particle Physics Latin American Network, European Union 7th Framework Program, Grant no. PIRSES-2009GA-246806; and UNESCO.

\section{Appendix A.}

A. Aab ${ }^{\mathrm{ar}}$, P. Abreu ${ }^{\text {bn }}$, M. Aglietta ${ }^{\mathrm{bc}}$, E.J. Ahn ${ }^{\mathrm{ce}}$, I. Al Samarai ${ }^{\mathrm{ad}}$, J.N. Albert ${ }^{\mathrm{ae}}$, I.F.M. Albuquerque $^{\mathrm{r}}$, I. Allekotte ${ }^{\mathrm{b}}$, J. Allen $^{\mathrm{ch}}$, P. Allison $^{\mathrm{cj}}$, A. Almela ${ }^{1, i}, J$. Alvarez Castillo ${ }^{\text {bg }}$, J. Alvarez-Muñiz ${ }^{\text {bx }}$, R. Alves Batista ${ }^{a q}$, M. Ambrosio ${ }^{\text {at }}$, A. Aminaei ${ }^{\text {bh }}$, L. Anchordoqui ${ }^{\text {cd }}$, S. Andringa ${ }^{\text {bn }}$,

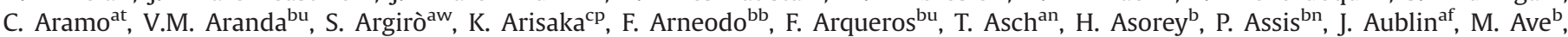
M. Avenier ${ }^{\mathrm{ag}}$, G. Avila ${ }^{\mathrm{k}}$, N. Awal ${ }^{\mathrm{ch}}$, A.M. Badescu ${ }^{\mathrm{br}}$, M. Balzer ${ }^{\mathrm{al}}$, K.B. Barber ${ }^{\mathrm{m}}$, A. Barbosa ${ }^{\mathrm{n}}$, N. Barenthien ${ }^{\mathrm{al}}$, M. Barkhausen ${ }^{\mathrm{ak}}$, J. Bäuml ${ }^{\mathrm{al}}$,

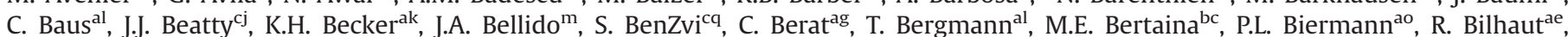
P. Billoir ${ }^{\text {af }}$, S.G. Blaess ${ }^{\mathrm{m}}$, M. Blanco ${ }^{\mathrm{af}}$, C. Bleve ${ }^{\mathrm{ax}}$, H. Blümer ${ }^{\mathrm{al}, \mathrm{am}}$, M. Boháčováab $^{\mathrm{ab}}, \mathrm{H}$. Bolz ${ }^{\mathrm{am}}$, D. Boncioli ${ }^{\mathrm{bb}}$, C. Bonifazi $^{\mathrm{x}}$, R. Bonino ${ }^{\mathrm{bc}}$, M. Boratav ${ }^{\text {af }}$, N. Borodai ${ }^{\text {bl }}$, F. Braccia ${ }^{\text {au }}$ J. Brack ${ }^{\text {cb }}$, I. Brancus $^{\text {bo }}$, A. Bridgeman ${ }^{\text {am }}$, P. Brogueira ${ }^{\text {bn }}$, W.C. Brown ${ }^{\text {cc }}$, P. Buchholz ${ }^{\text {ar }}$, A. Bueno ${ }^{\text {bw }}$, S. Buitink ${ }^{\text {bh }}$, M. Buscemi ${ }^{\text {at }}$, K.S. Caballero-Mora ${ }^{\text {be }, 8}$ B. Caccianiga ${ }^{\text {as }}$, L. Caccianiga ${ }^{\text {af }}$, D. Camin ${ }^{\text {as }, 1}$, M. Candusso ${ }^{\text {au }}$, L. Caramete ${ }^{\text {ao }}$, R. Caruso ${ }^{\text {av }}$,

\footnotetext{
7 Deceased.

${ }^{8}$ Now at Konan University.
} 


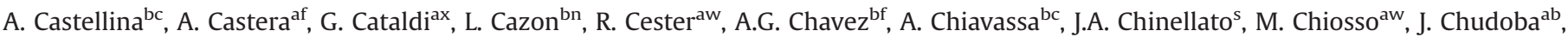
M. Cilmo ${ }^{\text {at }}$, P.D.J. Clark ${ }^{\text {by }}$, R.W. Clay ${ }^{\mathrm{m}}$, G. Cocciolo ${ }^{\mathrm{ax}}$, R. Colalillo ${ }^{\mathrm{at}}$, A. Coleman ${ }^{\mathrm{ck}}$, L. Collica ${ }^{\mathrm{as}}$, E. Colombo ${ }^{\mathrm{h}}$, S. Colonges ${ }^{\mathrm{aj}}$, M.R. Coluccia ${ }^{\mathrm{ax}}$, R. Conceição ${ }^{\text {bn }}$, F. Contreras ${ }^{\mathrm{j}}$, M.J. Cooper ${ }^{\mathrm{m}}$, J. Coppens ${ }^{\mathrm{bh}}$, A. Cordier ${ }^{\mathrm{ae}}$, B. Courty ${ }^{\mathrm{aj}}$, S. Coutu ${ }^{\text {ck }}$, C.E. Covault ${ }^{\mathrm{bz}}$, J. Cronin ${ }^{\mathrm{cl}}$, A. Curutiu ${ }^{\mathrm{ao}}$, R. Dallier ${ }^{\mathrm{a}, \mathrm{ah}}$, B. Daniel ${ }^{\mathrm{s}}$, S. Dasso ${ }^{\mathrm{f}, \mathrm{d}}$, K. Daumiller ${ }^{\mathrm{am}}$, B.R. Dawson ${ }^{\mathrm{m}}$, R.M. de Almeida ${ }^{\mathrm{y}}$, M. De Domenico ${ }^{\text {av }}$, C. De Donato ${ }^{\text {as }}$, S.J. de Jong ${ }^{\text {bh,bj }}$, J.R. T. de Mello Neto ${ }^{\mathrm{x}}$, I. De Mitri ${ }^{\mathrm{ax}}$, J. de Oliveira ${ }^{\mathrm{y}}$, V. de Souza ${ }^{\mathrm{q}}$, K.D. de Vries ${ }^{\mathrm{bi}}$, L. del Peral ${ }^{\mathrm{bv}}$, O. Deligny ${ }^{\text {ad }}$, H. Dembinski ${ }^{\text {am }}$, N. Dhital ${ }^{\text {cg }}$, C. Di Giulio $^{\text {au }}$, A. Di Matteo ${ }^{\text {ay }}$, J.C. Diaz ${ }^{\text {cg }}$, M.L. Díaz Castros, F. Diogo ${ }^{\text {bn }}$, C. Dobrigkeit ${ }^{\mathrm{s}}$, W. Docters ${ }^{\mathrm{bi}}$, J.C. D'Olivo ${ }^{\text {bg }}$, P. Dolron ${ }^{\text {bh }}$, A. Dorofeev $^{\text {cb }}$,

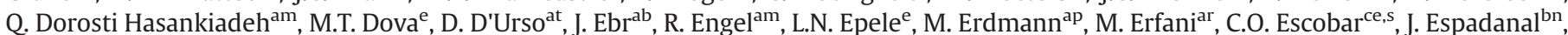
A. Etchegoyen ${ }^{\mathrm{i}, \mathrm{l}}$, P. Facal San Luis ${ }^{\mathrm{cl}}$, H. Falcke ${ }^{\mathrm{bh}, \mathrm{bk}, \mathrm{bj}}$, K. Fang ${ }^{\mathrm{cl}}$, G. Farrar ${ }^{\mathrm{ch}}$, A.C. Fauth ${ }^{\mathrm{s}}$, N. Fazzini ${ }^{\mathrm{ce}}$, A.P. Ferguson $^{\mathrm{bz}}$, M. Fernandes ${ }^{\mathrm{x}}$, A. Ferrero ${ }^{\mathrm{h}}$, B. Fick ${ }^{\mathrm{cg}}$, J.M. Figueira ${ }^{\mathrm{i}}$, A. Filevich ${ }^{\mathrm{i}}$, A. Filipčič ${ }^{\text {bs,bt }}$, B.D. Fox ${ }^{\mathrm{cm}}$, E.D. Fraenkel ${ }^{\text {bi }}$, O. Fratu ${ }^{\text {br }}$, M.M. Freire ${ }^{\mathrm{g}}$, U. Fröhlich ${ }^{\mathrm{ar}}$, B. Fuchs ${ }^{\mathrm{al}}$, W. Fulgione ${ }^{\mathrm{bc}}$,

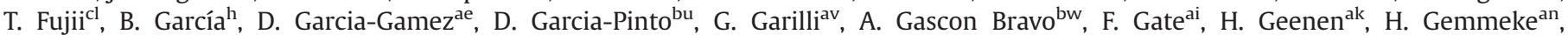

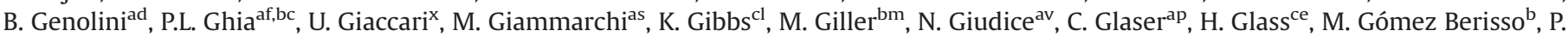
F. Gómez Vitale ${ }^{\mathrm{k}}$, P. Gonçalves ${ }^{\mathrm{bn}}$, J.G. Gonzalez ${ }^{\mathrm{al}}$, N. González ${ }^{\mathrm{i}}$, B. Gookinn ${ }^{\mathrm{cb}}$, D. Góra ${ }^{\mathrm{al}, \mathrm{bl}}$, J. Gordon ${ }^{\mathrm{cj}}$, A. Gorgi ${ }^{\mathrm{bc}}$, P. Gorham ${ }^{\mathrm{cm}}$, W. Gotink ${ }^{\mathrm{bj}}$, P. Gouffon ${ }^{\mathrm{r}}$, S. Grebe ${ }^{\mathrm{bh}, \mathrm{bj}}$, N. Griffith ${ }^{\mathrm{cj}}$, A.F. Grillo ${ }^{\mathrm{bb}}$, T.D. Grubb ${ }^{\mathrm{m}}$, J. Grygar ${ }^{\mathrm{ab}}$, N. Guardone ${ }^{\mathrm{av}}$, F. Guarino ${ }^{\mathrm{at}}$, G.P. Guedes ${ }^{\mathrm{t}}$, L. Guglielmi ${ }^{\mathrm{aj}}$,

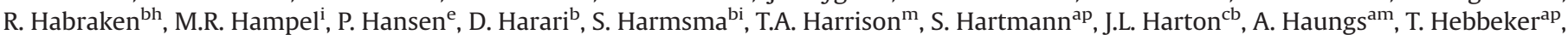
D. Heck ${ }^{\mathrm{am}}$, P. Heimann ${ }^{\mathrm{ar}}$, A.E. Herve ${ }^{\mathrm{am}}$, G.C. Hill ${ }^{\mathrm{m}}$, C. Hojvat ${ }^{\mathrm{ce}}$, N. Hollon ${ }^{\mathrm{cl}}$, E. Holt ${ }^{\mathrm{am}}$, P. Homola ${ }^{\text {ak }}$, J.R. Hörandel ${ }^{\text {bh,bj }}$, A. Horneffer ${ }^{\text {bh }}$,

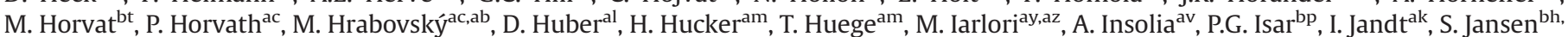
bj, C. Jarne ${ }^{\mathrm{e}}$, J.A. Johnsen ${ }^{\text {ca }}$, M. Josebachuili ${ }^{\mathrm{i}}$, A. Kääpäak ${ }^{\text {ak }}$ O. Kambeitz ${ }^{\text {al }}$, K.H. Kampert ${ }^{\text {ak, }}{ }^{*}$, P. Kasper ${ }^{\text {ce }}$, I. Katkov ${ }^{\text {al }}$, B. Kégl ${ }^{\text {ae }}$, B. Keilhauer ${ }^{\text {am }}$, A. Keivanick ${ }^{\text {, }}$ J. Kelley ${ }^{\text {bh }}$, E. Kemp ${ }^{\text {s }}$, R.M. Kieckhafer ${ }^{\text {cg }}$, H.O. Klages ${ }^{\text {am }}$, M. Kleifges ${ }^{\text {an }}$, J. Kleinfeller ${ }^{\mathrm{j}}$, J. Knapp $^{\text {by }}$, A. Kopmann ${ }^{\text {an }}$, R. Krause ${ }^{\text {ap }}$, N. Krohm ${ }^{\text {ak }}$, O. Krömer ${ }^{\text {an }}$, D. Kuempel ${ }^{\text {ap }}$, N. Kunka ${ }^{a}$, D. LaHurd ${ }^{\text {bz }}$, L. Latronico ${ }^{\text {bc }}$, R. Lauer ${ }^{\text {co }}$, M. Lauscher ${ }^{\text {ap }}$, P. Lautridou ${ }^{\text {ai }}$, S. Le Coz ${ }^{\text {ag }}$, M.S.A. B. Leão ${ }^{\circ}$, D. Lebrun ${ }^{\mathrm{ag}}$, P. Lebrun ${ }^{\mathrm{ce}}$, M.A. Leigui de Oliveira ${ }^{\mathrm{w}}$, A. Letessier-Selvon ${ }^{\mathrm{af}}$, I. Lhenry-Yvon ${ }^{\mathrm{ad}}$, K. Link ${ }^{\mathrm{al}}$, R. López ${ }^{\mathrm{bd}}$, A. López Casado ${ }^{\mathrm{bx}}$,

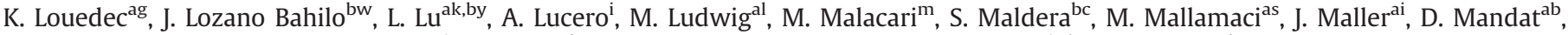

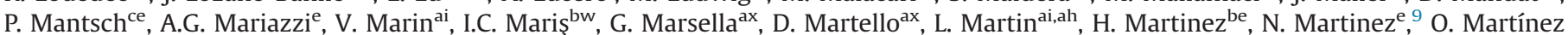
Bravo $^{\text {bd }}$, D. Martraire ${ }^{\text {ad }}$, J.J. Masías Meza ${ }^{\mathrm{d}}$, H.J. Mathes ${ }^{\mathrm{am}}$, S. Mathys ${ }^{\mathrm{ak}}$, J. Matthews ${ }^{\mathrm{cf}}$, J.A.J. Matthews ${ }^{\mathrm{co}}$, G. Matthiae ${ }^{\mathrm{au}}$, D. Maurel $^{\mathrm{al}}$, D. Maurizion ${ }^{n}$ E. Mayotte ${ }^{\text {ca }}$, P.O. Mazur ${ }^{\text {ce }}$, C. Medina ${ }^{\text {ca }}$, G. Medina-Tanco ${ }^{\text {bg }}$, R. Meissner ${ }^{\text {ap }}$, M. Melissas ${ }^{\text {al }}$, V.B.B. Mello ${ }^{\mathrm{x}}$, D. Melo, E. Menichettiaw, A. Menshikov ${ }^{\text {an }}$, S. Messina ${ }^{\text {bi }}$, R. Meyhandan ${ }^{\mathrm{cm}}$, S. Mićanovićc ${ }^{\mathrm{z}}$, M.I. Micheletti ${ }^{\mathrm{g}}$, L. Middendorf ${ }^{\mathrm{ap}}$, I.A. Minaya ${ }^{\text {bu }}$, L. Miramontias, B. Mitrica ${ }^{\text {bo }}$, L. Molina-Bueno ${ }^{\text {bw }}$, S. Mollerach ${ }^{\mathrm{b}}$, M. Monasor ${ }^{\mathrm{cl}}$, F. Montanet ${ }^{\mathrm{ag}}$, C. Morello ${ }^{\mathrm{bc}}$, M. Mostafá $^{\mathrm{ck}}$, C.A. Moura $^{\mathrm{w}}$,

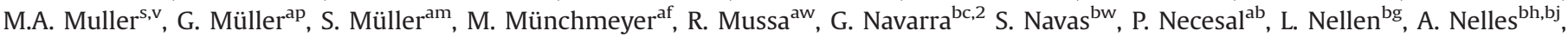

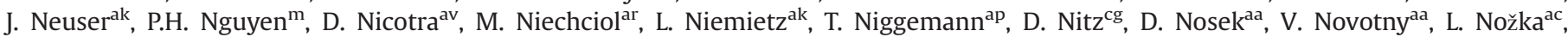

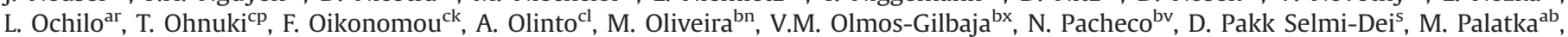

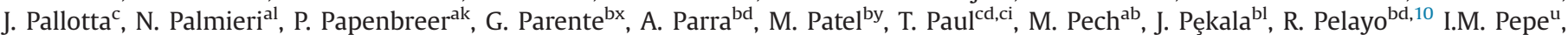

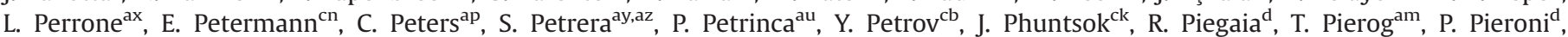
M. Pimenta ${ }^{\text {bn }}$, V. Pirronello ${ }^{\text {av }}$, M. Platino ${ }^{\text {i }}$, M. Plum ${ }^{\text {ap }}$, A. Porcelliam, C. Porowski ${ }^{\text {bl }}$, T. Porter ${ }^{\text {cf }}$, J. Pouryamout $^{\text {ak }}$, J. Pouthas ${ }^{\text {ad }}$, R.R. Prado ${ }^{\mathrm{q}}$, P. Privitera ${ }^{\mathrm{cl}}$, M. Prouza ${ }^{\mathrm{ab}}$, C.L. Pryke ${ }^{\mathrm{cl}}$, V. Purrello ${ }^{\mathrm{b}}$, E.J. Quel ${ }^{\mathrm{c}}$, S. Querchfeld ${ }^{\mathrm{ak}}$, S. Quinn ${ }^{\mathrm{bz}}$, R. Randriatoamanana ${ }^{\text {af }}$, J. Rautenberg ${ }^{\mathrm{ak}}$, O. Ravel $^{\mathrm{ai}}$,

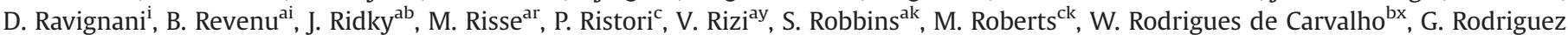
Fernandez ${ }^{\mathrm{au}}$, J. Rodriguez Rojo ${ }^{\mathrm{j}}$, M.D. Rodríguez-Frías ${ }^{\mathrm{bv}}$, D. Rogozin ${ }^{\mathrm{am}}$, G. Ros ${ }^{\mathrm{bv}}$, J. Rosado ${ }^{\mathrm{bu}}$, T. Rossler $^{\mathrm{ac}}$, M. Roth ${ }^{\mathrm{am}}$, E. Roulet ${ }^{\mathrm{b}}$, A.C. Rovero, $^{\mathrm{f}}$,

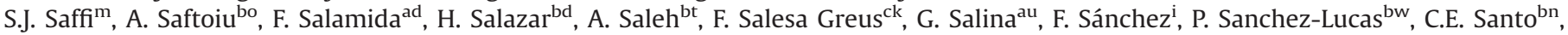
E. Santos ${ }^{\text {s }}$, E.M. Santos ${ }^{\text {r }}$, F. Sarazin ${ }^{\text {ca }}$, B. Sarkar ${ }^{\mathrm{ak}}$, R. Sarmento ${ }^{\text {bn }}$, R. Sato ${ }^{\mathrm{j}}$, N. Scharf ${ }^{\text {ap }}$, V. Scherini ${ }^{\text {ax }}$, H. Schieler $^{\text {am }}$, P. Schiffer ${ }^{\text {aq }}$, A. Schmidt ${ }^{\text {an }}$, D. Schmidt ${ }^{\text {am }}$, O. Scholten ${ }^{\text {bi,6 }}$ H. Schoorlemmer ${ }^{\text {cm,bh,bj }}$, P. Schovánek ${ }^{\text {ab }}$, F. Schreuder ${ }^{\text {bi }}$, F.G. Schröder ${ }^{\text {am }}$, A. Schulz ${ }^{\text {am }}$, J. Schulz ${ }^{\text {bh }}$, F. Schüssler ${ }^{\text {am }}$, J. Schumacher ${ }^{\text {ap }}$, S.J. Sciutto ${ }^{\mathrm{e}}$, A. Segreto ${ }^{\text {ba }}$, G. Sequeiros ${ }^{\text {aw }}$, M. Settimo ${ }^{\text {af }}$, A. Shadkam ${ }^{\text {cf }}$, R.C. Shellard ${ }^{\text {, }}$, I. Sidelnik ${ }^{\text {b }}$, G. Sigl ${ }^{\mathrm{aq}}$, O. Sima ${ }^{\mathrm{bq}}$, A. Śmiałkowski ${ }^{\mathrm{bm}}$, R. Šmída ${ }^{\mathrm{am}}$, A.G.K. Smith ${ }^{\mathrm{m}}$, G.R. Snow ${ }^{\mathrm{cn}}$, P. Sommers ${ }^{\text {ck }}$, J. Sorokin ${ }^{\mathrm{m}}$, R. Speelman ${ }^{\mathrm{bi}}$, H. Spinka ${ }^{\mathrm{ce}}$,

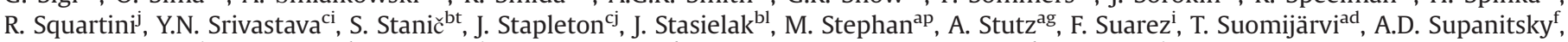
M.S. Sutherland ${ }^{\mathrm{cj}}$, M. Sutter ${ }^{\mathrm{al}}$, J. Swain ${ }^{\mathrm{ci}}$, Z. Szadkowski ${ }^{\mathrm{bm}}$, M. Szuba $^{\mathrm{am}}$, O.A. Taborda ${ }^{\mathrm{b}}$, A. Tapia ${ }^{\mathrm{i}}$, D. Tcherniakhovski ${ }^{\text {an }}$, A. Tepe ${ }^{\text {ar }}$, V.M. Theodoros, C. Timmermans ${ }^{\text {bj,bh }}$, W. Tkaczyk ${ }^{\text {bm, } 2}$, C.J. Todero Peixoto ${ }^{p}$, G. Toma ${ }^{\text {bo }}$, L. Tomankova ${ }^{\text {am }}$, B. Tomé ${ }^{\text {bn }}$, A. Tonachini ${ }^{\text {aw }}$,

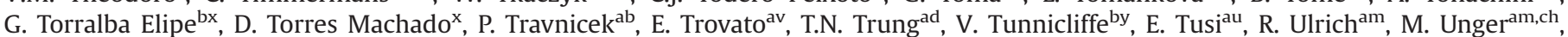
M. Urban ap , J.F. Valdés Galicia ${ }^{\mathrm{bg}}$, I. Valiño ${ }^{\mathrm{bx}}$, L. Valore ${ }^{\mathrm{at}}$, G. van Aar $^{\mathrm{bh}}$, P. van Bodegom ${ }^{\mathrm{m}}$, A.M. van den Berg ${ }^{\mathrm{bi}}$, S. van Velzen ${ }^{\mathrm{bh}}$, A. van Vliet ${ }^{\mathrm{aq}}$, E. Varela ${ }^{\text {bd }}$, B. Vargas Cárdenas ${ }^{\text {bg }}$, D.M. Varnav ${ }^{\text {bi }}$, G. Varner $^{\mathrm{cm}}$, R. Vasquez $^{\mathrm{x}}$, J.R. Vázquez ${ }^{\text {bu }}$, R.A. Vázquez ${ }^{\text {bx }}$, D. Veberič ${ }^{\mathrm{am}}$, H. Verkooijen ${ }^{\mathrm{bj}}$,

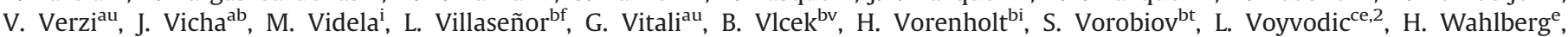

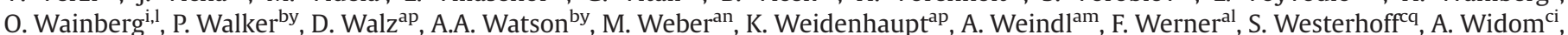

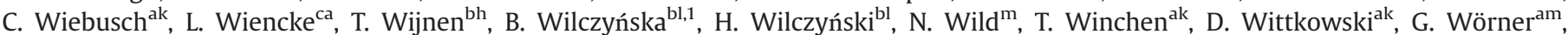
B. Wundheiler ${ }^{\mathrm{i}}$, S. Wykes ${ }^{\mathrm{bh}}$, T. Yamamoto ${ }^{\mathrm{cl}, 11}$, T. Yapici $^{\mathrm{cg}}$, G. Yuan $^{\mathrm{cf}}$, A. Yushkov $^{\mathrm{ar}}$, B. Zamorano $^{\text {bw }}$, E. Zas $^{\mathrm{bx}}$, D. Zavrtanik $^{\mathrm{bt}, \mathrm{bs}}$, M. Zavrtanik $^{\mathrm{bs}, \mathrm{bt}}$, A. Zepeda ${ }^{\text {be, } 12}$, J. Zhou ${ }^{\text {cl }}$, Y. Zhu ${ }^{\text {an }}$, M. Zimbres Silva , B. Zimmermann ${ }^{\text {an }}$, M. Ziolkowski ${ }^{\text {ar }}$, F. Zuccarello ${ }^{\text {av }}$

${ }^{\mathrm{b}}$ Centro Atómico Bariloche and Instituto Balseiro San Carlos de Bariloche, Argentina ${ }^{\mathrm{c} C e n t r o ~ d e ~ I n v e s t i g a c i o n e s ~ e n ~ L a ́ s e r e s ~ y ~ A p l i c a c i o n e s, ~ C I T E D E F ~ a n d ~ C O N I C E T, ~ A r g e n t i n a ~}$

${ }^{\mathrm{d}}$ Departamento de Física, FCEyN, Universidad de Buenos Aires and CONICET, Argentina

' IFLP, Universidad Nacional de La Plata and CONICET, La Plata, Argentina

fInstituto de Astronomía y Física del Espacio (IAFE, CONICET-UBA), Buenos Aires, Argentina

${ }^{\mathrm{g}}$ Instituto de Física de Rosario (IFIR) CONICET/U.N.R. and Facultad de Ciencias Bioquímicas y Farmacéuticas U.N.R., Rosario, Argentina

${ }^{\mathrm{h}}$ Instituto de Tecnologías en Detección y Astropartículas (CNEA, CONICET, UNSAM), and Universidad Tecnológica Nacional Facultad Regional Mendoza (CONICET/CNEA), Argentina

instituto de Tecnologías en Detección y Astropartículas (CNEA, CONICET, UNSAM), Buenos Aires, Argentina

\footnotetext{
9 Also at the Universidad Autonoma de Chiapas; on leave of absence from Cinvestav.

${ }^{10}$ Now at Unidad Profesional Interdisciplinaria de Ingeniería y Tecnologías Avanzadas del IPN, México, D.F., Mexico.

11 Now at Universidad Autónoma de Chiapas, Tuxtla Gutiérrez, Chiapas, Mexico.

12 Also at Vrije Universiteit Brussels, Belgium.
} 
${ }^{\mathrm{j} O b s e r v a t o r i o ~ P i e r r e ~ A u g e r, ~ M a l a r g u ̈ e, ~ A r g e n t i n a ~}$

kObservatorio Pierre Auger and Comisión Nacional de Energía Atómica, Malargüe, Argentina

${ }^{1}$ Universidad Tecnológica Nacional Facultad Regional Buenos Aires, Buenos Aires, Argentina

${ }^{\mathrm{m}}$ University of Adelaide, Adelaide, S.A., Australia

${ }^{\mathrm{n}}$ Centro Brasileiro de Pesquisas Físicas, Rio de Janeiro, RJ, Brazil

${ }^{\circ}$ Faculdade Independente do Nordeste, Vitória da Conquista, Brazil

${ }^{\mathrm{p}}$ Universidade de São Paulo, Escola de Engenharia de Lorena, Lorena, SP, Brazil

${ }^{\mathrm{q}}$ Universidade de São Paulo, Instituto de Física de São Carlos, São Carlos, SP, Brazil

${ }^{\mathrm{r}}$ Universidade de São Paulo, Instituto de Física, São Paulo, SP, Brazil

${ }^{\mathrm{s}}$ Universidade Estadual de Campinas, IFGW, Campinas, SP, Brazil

${ }^{\mathrm{t}}$ Universidade Estadual de Feira de Santana, Brazil

"Universidade Federal da Bahia, Salvador, BA, Brazil

${ }^{\vee}$ Universidade Federal de Pelotas, Pelotas, RS, Brazil

${ }^{w}$ Universidade Federal do ABC, Santo André, SP, Brazil

${ }^{\mathrm{x}}$ Universidade Federal do Rio de Janeiro, Instituto de Física, Rio de Janeiro, RJ, Brazil

${ }^{\mathrm{y}}$ Universidade Federal Fluminense, EEIMVR, Volta Redonda, RJ, Brazil

${ }^{\mathrm{z}}$ Rudjer Bošković Institute, 10000 Zagreb, Croatia

${ }^{\text {aa }}$ Charles University, Faculty of Mathematics and Physics, Institute of Particle and Nuclear Physics, Prague, Czech Republic

${ }^{\mathrm{ab}}$ Institute of Physics of the Academy of Sciences of the Czech Republic, Prague, Czech Republic

${ }^{a c}$ Palacky University, RCPTM, Olomouc, Czech Republic

ad Institut de Physique Nucléaire d'Orsay (IPNO), Université Paris 11, CNRS-IN2P3, France

ae Laboratoire de l'Accélérateur Linéaire (LAL), Université Paris 11, CNRS-IN2P3, France

${ }^{a f}$ Laboratoire de Physique Nucléaire et de Hautes Energies (LPNHE), Universités Paris 6'et Paris 7, CNRS-IN2P3, Paris, France

${ }^{\mathrm{ag}}$ Laboratoire de Physique Subatomique et de Cosmologie (LPSC), Université Grenoble-Alpes, CNRS/IN2P3,France

${ }^{\text {ah }}$ Station de Radioastronomie de Nançay, Observatoire de Paris, CNRS/INSU, France

ai SUBATECH, École des Mines de Nantes, CNRS-IN2P3, Université de Nantes, France

${ }^{\mathrm{aj}}$ Laboratoire AstroParticule et Cosmologie, Université Paris 7, IN2P3/CNRS, Paris, France

${ }^{\mathrm{ak}}$ Bergische Universität Wuppertal, Wuppertal, Germany

${ }^{a l}$ Karlsruhe Institute of Technology Campus South Institut für Experimentelle Kernphysik (IEKP), Karlsruhe, Germany

${ }^{a m}$ Karlsruhe Institute of Technology Campus North Institut für Kernphysik, Karlsruhe, Germany

${ }^{a n}$ Karlsruhe Institute of Technology Campus North Institut für Prozessdatenverarbeitung und Elektronik, Karlsruhe, Germany

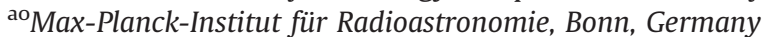

${ }^{\mathrm{ap}}$ RWTH Aachen University, III. Physikalisches Institut A, Aachen, Germany

${ }^{\mathrm{aq}}$ Universität Hamburg, Hamburg, Germany

${ }^{\mathrm{ar}}$ Universität Siegen, Siegen, Germany

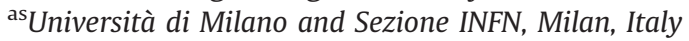

at Università di Napoli "Federico II" and Sezione INFN, Napoli, Italy

au Università di Roma II "Tor Vergata" and Sezione INFN, Roma, Italy

av Università di Catania and Sezione INFN,

${ }^{\mathrm{aw}}$ Università di Torino and Sezione INFN, Torino, Italy

${ }^{a x}$ Dipartimento di Matematica e Fisica "E. De Giorgi" dell'Università del Salento and Sezione INFN, Lecce, Italy

${ }^{a y}$ Dipartimento di Scienze Fisiche e Chimiche dell'Università dell'Aquila and INFN, Italy

${ }^{\mathrm{az}}$ Gran Sasso Science Institute (INFN), L'Aquila, Italy

ba Istituto di Astrofisica Spaziale e Fisica Cosmica di Palermo (INAF), Palermo, Italy

${ }^{\mathrm{bb}}$ INFN, Laboratori Nazionali del Gran Sasso, Assergi (L'Aquila), Italy

${ }^{\mathrm{bc} O s s e r v a t o r i o ~ A s t r o f i s i c o ~ d i ~ T o r i n o ~(I N A F), ~ U n i v e r s i t a ̀ ~ d i ~ T o r i n o ~ a n d ~ S e z i o n e ~ I N F N, ~ T o r i n o, ~ I t a l y ~}$

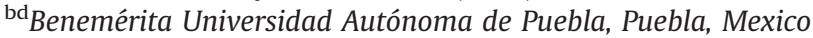

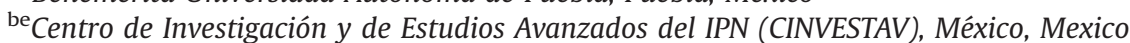

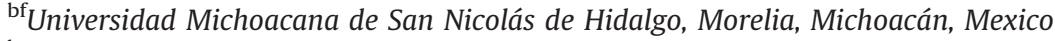

bg Universidad Nacional Autónoma de México, México, D.F., Mexico

${ }^{\mathrm{bh}}$ IMAPP, Radboud University Nijmegen, Netherlands

${ }^{b i}$ KVI Center for Advanced Radiation Technology, University of Groningen, Netherlands

${ }^{b j}$ NIKHEF, Science Park, Amsterdam, Netherlands

${ }^{\mathrm{bk}}$ ASTRON, Dwingeloo, Netherlands

${ }^{\mathrm{bl}}$ Institute of Nuclear Physics PAN, Krakow, Poland

bmUniversity of Łódź, Łódź, Poland

${ }^{b n}$ Laboratório de Instrumentação e Física Experimental de Partículas LIP and Instituto Superior Técnico IST, Universidade de Lisboa UL, Portugal

bo'Horia Hulubei' National Institute for Physics and Nuclear Engineering, Bucharest-Magurele, Romania

${ }^{\mathrm{bp}}$ Institute of Space Sciences, Bucharest, Romania

${ }^{\mathrm{bq}}$ University of Bucharest, Physics Department, Romania

${ }^{\mathrm{br}}$ University Politehnica of Bucharest, Romania

${ }^{\mathrm{bs}}$ Experimental Particle Physics Department, J. Stefan Institute, Ljubljana, Slovenia

${ }^{b t}$ Laboratory for Astroparticle Physics, University of Nova Gorica, Slovenia

bu Universidad Complutense de Madrid, Madrid, Spain

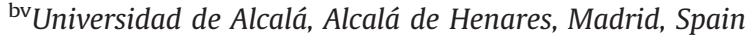


${ }^{\mathrm{bw}}$ Universidad de Granada and C.A.F.P.E., Granada, Spain

${ }^{\mathrm{bx}}$ Universidad de Santiago de Compostela, Spain

by School of Physics and Astronomy, University of Leeds, United Kingdom

${ }^{\mathrm{bz}}$ Case Western Reserve University, Cleveland, $\mathrm{OH}$, USA

${ }^{\mathrm{ca} C o l o r a d o}$ School of Mines, Golden, CO, USA

${ }^{\mathrm{cb} C o l o r a d o}$ State University, Fort Collins, CO, USA

${ }^{\mathrm{cc} C o l o r a d o}$ State University, Pueblo, CO, USA

${ }^{\mathrm{cd} D e p a r t m e n t}$ of Physics and Astronomy, Lehman College, City University of New York, New York, USA

${ }^{\mathrm{ce}}$ Fermilab, Batavia, IL, USA

${ }^{\mathrm{cf}}$ Louisiana State University, Baton Rouge, LA, USA

${ }^{\mathrm{cg}}$ Michigan Technological University, Houghton, MI, USA

${ }^{\mathrm{ch}}$ New York University, New York, NY, USA

${ }^{\mathrm{ci}}$ Northeastern University, Boston, MA, USA

${ }^{\mathrm{cj} O h i o ~ S t a t e}$ University, Columbus, $\mathrm{OH}, \mathrm{USA}$

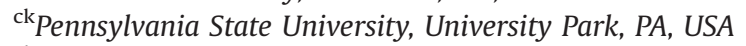

${ }^{\mathrm{cl}}$ University of Chicago, Enrico Fermi Institute, Chicago, IL, USA

${ }^{\mathrm{cm}}$ University of Hawaii, Honolulu, HI, USA

${ }^{\mathrm{c}}$ University of Nebraska, Lincoln, NE, USA

${ }^{\mathrm{co}}$ University of New Mexico, Albuquerque, NM, USA

${ }^{\mathrm{cp}}$ University of California, Los Angeles (UCLA), Los Angeles, CA, USA

${ }^{\mathrm{cq}}$ University of Wisconsin, Madison, WI, USA

\section{References}

[1] B. Rossi, Supplemento a la Ricerca Scientifica 1 (1934) 579.

[2] K. Schmeiser, W. Bothe, Annals of Physics 424 (1938) 161. http://dx.doi.org/ 10.1002/andp.19384240119.

[3] W. Kolhörster, I. Matthes, E. Weber, Gekoppelte Höhenstrahlen, Naturwissenschaften 26 (1938) 576.

[4] P. Auger, R. Maze, A.F. Robley, Comptes Rendus 208 (1939) 1641.

[5] P. Auger, P. Ehrenfest, R. Maze, J. Daudin, A.F. Robley, Reviews of Modern Physics 11 (1939) 288. http://dx.doi.org/10.1103/RevModPhys.11.288.

[6] J. Linsley, Physical Review Letters 10 (1963) 146. http://dx.doi.org/10.1103/ PhysRevLett.10.146.

[7] B. Afanasiev, et al., Recent results from Yakutsk experiment, in: M. Nagano (Ed.), Proceedings of the Tokyo Workshop on Techniques for the Study of the Extremely High Energy Cosmic Rays, Institute for Cosmic Ray Research, University of Tokyo, Tokyo, Japan, 1993, p. 35.

[8] M. Lawrence, R. Reid, A. Watson, Journal of Physics G 17 (1991) 733. http: //dx.doi.org/10.1088/0954-3899/17/5/019.

[9] M. Takeda, N. Sakaki, K. Honda, M. Chikawa, M. Fukushima, et al., Astroparticle Physics 19 (2003) 447. http://dx.doi.org/10.1016/S0927-6505(02) 00243-8 (arxiv:astro-ph/0209422).

[10] D. Bird, S. Corbato, H. Dai, J. Elbert, K. Green, et al., Astrophysical Journal 441 (1995) 144. http://dx.doi.org/10.1086/175344.

[11] A. Schulz, Measurement of the energy spectrum of cosmic rays above $3 \times$ $10^{17} \mathrm{eV}$ with the Pierre Auger Observatory [171], 2013, p. 27 (arXiv:1307. 5059).

[12] J. Abraham, et al., Physical Review Letters 101 (2008) 061101. http://dx.doi. org/10.1103/PhysRevLett.101.061101 (arXiv:0806.4302).

[13] P. Abreu, et al., Astroparticle Physics 34 (2011) 627. http://dx.doi.org/10.1016/ j.astropartphys.2010.12.007 (arXiv:1103.2721).

[14] I. Sidelnik, Measurement of the first harmonic modulation in the right ascension distribution of cosmic rays detected at the Pierre Auger Observatory: towards the detection of dipolar anisotropies over a wide energy range [171], 2013, p. 56 (arXiv:1307.5059).

[15] R. de Almeida, Constraints on the origin of cosmic rays from large scale anisotropy searches in data of the Pierre Auger Observatory [171], 2013, p. 60 (arXiv:1307.5059).

[16] P. Abreu, et al., Astrophysical Journal 762 (2012) L13. http://dx.doi.org/ 10.1088/2041-8205/762/1/L13 (arXiv:1212.3083).

[17] J. Abraham, et al., Astroparticle Physics 29 (2008) 188. http://dx.doi.org/ 10.1016/j.astropartphys.2008.06.004, 10.1016/j.astropartphys.2008.01.002 (arXiv:0712.2843).

[18] J. Abraham, et al., Science 318 (2007) 938. http://dx.doi.org/10.1126/ science.1151124 (arxiv:0711.2256).

[19] P. Abreu, et al., Physical Review Letters 109 (2012) 062002. http://dx.doi.org/ 10.1103/PhysRevLett.109.062002 (arXiv:1208.1520).

[20] T. Csörgö, et al., Progress of Theoretical Physics Supplement 193 (2012) 180. http://dx.doi.org/10.1143/PTPS.193.180 (arXiv:1204.5689).

[21] J. Abraham, et al., Physical Review Letters 104 (2010) 091101. http://dx.doi. org/10.1103/PhysRevLett.104.091101 (arXiv:1002.0699).

[22] P. Abreu, et al., Journal of Cosmology and Astroparticle Physics 1302 (2013) 026. http://dx.doi.org/10.1088/1475-7516/2013/02/026 (arXiv:1301.6637).

[23] A. Aab, et al., Phys. Rev. D 91, 032003 (2015), http://dx.doi.org/10.1103/ PhysRevD.91.032003, (arXiv:1408.1421).

[24] A. Aab, et al., Physical Review D 90 (2014) 012012. http://dx.doi.org/10.1103/ PhysRevD.90.012012 (arXiv:1407.5919).

[25] M. Settimo, Proceedings of Science (Photon 2013) (2013) 062. URL 〈http:// pos.sissa.it/archive/conferences/192/062/Photon202013_062.pdf).

[26] A. Aab, et al., Astrophysical Journal 789 (2014) 160. http://dx.doi.org/10.1088/ 0004-637X/789/2/160 (arXiv:1406.2912).

[27] P. Pieroni, Ultra-high energy neutrinos at the Pierre Auger Observatory [171], 2013, p. 76 (arXiv:1307.5059).

[28] P. Abreu, et al., Astrophysical Journal 755 (2012) L4. http://dx.doi.org/ 10.1088/2041-8205/755/1/L4 (arXiv:1210.3143).

[29] P. Abreu, et al., Advances in High Energy Physics, 2013 (2013) 708680. http:// dx.doi.org/10.1155/2013/708680.

[30] P. Abreu, et al., Astrophysical Journal 760 (2012) 148. http://dx.doi.org/ 10.1088/0004-637X/760/2/148 (arXiv:1211.4901).

[31] A. Aab, et al., Astrophysical Journal 789 (2014) L34. http://dx.doi.org/10.1088/ 2041-8205/789/2/L34 (arXiv:1406.4038).

[32] The Pierre Auger Collaboration, Design Report 〈http://www.auger.org/techni cal_info/design_report.html $>$ (accessed 16.01.15).

[33] J. Abraham, et al., Nuclear Instruments and Methods in Physics Research Section A 523 (2004) 50. http://dx.doi.org/10.1016/j.nima.2003.12.012.

[34] P. Sommers, Astroparticle Physics 3 (1995) 349. http://dx.doi.org/10.1016/ 0927-6505(95)00013-7.

[35] B. Dawson, H. Dai, P. Sommers, S. Yoshida, Astroparticle Physics 5 (1996) 239. http://dx.doi.org/10.1016/0927-6505(96)00024-2.

[36] J. Abraham, et al., Nuclear Instruments and Methods in Physics Research Section A 613 (2010) 29. http://dx.doi.org/10.1016/j.nima.2009.11.018 (arXiv:1111.6764).

[37] P. Abreu, et al., Astroparticle Physics 34 (2011) 368. http://dx.doi.org/10.1016/ j.astropartphys.2010.10.001 (arXiv:1010.6162).

[38] J. Abraham, et al., Physics Letters B 685 (2010) 239. http://dx.doi.org/10.1016/ j.physletb.2010.02.013 (arXiv:1002.1975).

[39] J. Hersil, I. Escobar, D. Scott, G. Clark, S. Olbert, Physical Review Letters 6 (1961) 22. http://dx.doi.org/10.1103/PhysRevLett.6.22.

[40] C. Bonifazi, Nuclear Physics Proceedings Supplements 190 (2009) 20. http: //dx.doi.org/10.1016/j.nuclphysbps.2009.03.063 (arXiv:0901.3138).

[41] B. Dawson, Hybrid performance of the Pierre Auger Observatory, in: Proceedings of the 30th International Cosmic Ray Conference (ICRC), Merida, Mexico, 2007 (arXiv:0706.1105).

[42] I. Allekotte, et al., Nuclear Instruments and Methods in Physics Research Section A 586 (2008) 409. http://dx.doi.org/10.1016/j.nima.2007.12.016 (arXiv:0712.2832).

[43] X. Bertou, et al., Nuclear Instruments and Methods in Physics Research Section A 568 (2006) 839. http://dx.doi.org/10.1016/j.nima.2006.07.066.

[44] P. Allison, et al., Observing muon decays in water Cherenkov detectors at the Pierre Auger Observatory, in: Proceedings of the 29th International Cosmic Ray Conference (ICRC), Pune, India, vol. 8, 2005, pp. 299-302 (arXiv: astro-ph/0509238).

[45] R. Sato, Long term performance of the surface detectors of the Pierre Auger Observatory [89], 2011, p. 1 (arXiv:1107.4806).

[46] J. Rautenberg, Remote operation of the Pierre Auger Observatory [89], 2011, p. 5 (arXiv:1107.4806).

[47] J. Abraham, et al., Nuclear Instruments and Methods in Physics Research Section A 620 (2010) 227. http://dx.doi.org/10.1016/j.nima.2010.04.023 (arXiv:0907.4282).

[48] M. de Oliveira, V. de Souza, H. Reis, R. Sato, Nuclear Instruments and Methods in Physics Research Section A 522 (2004) 360. http://dx.doi.org/ 10.1016/j.nima.2003.11.409.

[49] J. Baeuml, Measurement of the optical properties of the Auger fluorescence telescopes [171], p. 2013, 15 (arXiv:1307.5059). 
[50] K. Becker, A. Behrmann, H. Geenen, S. Hartmann, K. Kampert, et al., Nuclear Instruments and Methods in Physics Research Section A 576 (2007) 301. http://dx.doi.org/10.1016/j.nima.2007.03.007.

[51] B. Fick, et al., Journal of Instrumentation 1 (2006) P11003. http://dx.doi.org/ 10.1088/1748-0221/1/11/P11003 (arXiv:astro-ph/0507334)

[52] J. Brack, R. Cope, A. Dorofeev, B. Gookin, J. Harton, et al., Journal of Instrumentation 8 (2013) P05014. http://dx.doi.org/10.1088/1748-0221/8/ 05/P05014 (arXiv:1305.1329).

[53] S. BenZvi, R. Cester, M. Chiosso, B. Connolly, A. Filipcic, et al., Nuclear Instruments and Methods in Physics Research Section A 574 (2007) 171 http://dx.doi.org/10.1016/j.nima.2007.01.094 (arXiv:astro-ph/0609063).

[54] S. Argiro, S. Barroso, J. Gonzalez, L. Nellen, T.C. Paul, et al., Nuclear Instruments and Methods in Physics Research Section A 580 (2007) 1485. http://dx.doi.org/10.1016/j.nima.2007.07.010 (arXiv:0707.1652).

[55] E. Harold, W. Means, XML in a Nutshell, O'Reilly Media, Sebastopol, CA, 2004 (ISBN 0-596-00764-7).

[56] Python 〈https://www.python.org/ (accessed 15.01.15).

[57] ROOT-Data Analysis Framework 〈https://root.cern.ch/drupal/〉 (accessed 15.01.15).

[58] S. Sciutto, AIRES: A System for Air Shower Simulations. User's Guide and Reference Manual, Version 2.2.0, 1999, Downloads and manuals to the software can be found at 〈http://www2.fisica.unlp.edu.ar/auger/aires/eg Aires.html (arXiv:astro-ph/9911331).

[59] D. Heck, G. Schatz, T. Thouw, J. Knapp, J. Capdevielle, CORSIKA: A Monte Carlo Code to Simulate Extensive Air Showers, Forschungszentrum Karlsruhe - Wissenschaftliche Berichte, Downloads and manuals to the software can be found at 〈https://web.ikp.kit.edu/corsika/〉.

[60] T. Bergmann, R. Engel, D. Heck, N. Kalmykov, S. Ostapchenko, et al., Astroparticle Physics 26 (2007) 420. http://dx.doi.org/10.1016/j.astropartphys.2006.08.005 (for further information contact the authors of CORSIKA, see url at [59]. astro-ph/0606564).

[61] H.-J. Drescher, G.R. Farrar, Physical Review D 67 (2003) 116001. http://dx.doi. org/10.1103/PhysRevD.67.116001 (Downloads and manuals to the software can be found at http://th.physik.uni-frankfurt.de/ rdrescher/SENECA/).

[62] Apache-Xerces 〈http://xerces.apache.org/〉 (accessed 15.01.15).

[63] XML-SCHEMA 〈http://www.w3.org/standards/xml/schema〉 (accessed 15.01.15).

[64] CppUnit - C ++ Port of JUnit 〈http://sourceforge.net/projects/cppunit/〉 (accessed 16.01.15).

[65] Buildbot-The Continuous Integration Framework 〈http://buildbot.net/〉 (accessed 16.01.15).

[66] CMake 〈http://www.cmake.org (accessed 16.01.15).

[67] Auger Package Environment 〈https://svn.auger.unam.mx/trac/projects/ape/〉 (accessed 16.01.15).

[68] B. Boehm, Software engineering economics, in: Prentice-Hall Advances in Computing Science \& Technology Series, 1981 (ISBN 0-13-822122-7).

[69] P. Abreu, et al., Nuclear Instruments and Methods in Physics Research Section A 635 (2011) 92. http://dx.doi.org/10.1016/j.nima.2011.01.049 (arXiv:1101. 4473).

[70] D. Kostunin, N. Budnev, O. Gress, A. Haungs, R. Hiller, et al., Nuclear Instruments and Methods in Physics Research Section A 742 (2014) 89. http://dx.doi.org/10.1016/j.nima.2013.10.070 (arXiv:1310.8477)

[71] A. Abeysekara, et al., The HAWC Gamma-Ray Observatory: Design, Calibration, and Operation, 2013, (arXiv:1310.0074).

[72] T. Ebisuzaki, G. Medina-Tanco, A. Santangelo, Advances in Space Research 53 (2014) 1499. http://dx.doi.org/10.1016/j.asr.2013.11.056.

[73] D. Ardouin, A. Belletoile, D. Charrier, R. Dallier, L. Denis, et al., International Journal of Modern PhysicsA21S1 (2006) 192. http://dx.doi.org/10.1142/ S0217751X0603360X.

[74] O. Wyszynski, A. Laszlo, A. Marcinek, T. Paul, R. Sipos, et al., Journal of Physics: Conference Series 396 (2012) 052076. http://dx.doi.org/10.1088/ 1742-6596/396/5/052076.

[75] R. Sipos, A. Laszlo, A. Marcinek, T. Paul, M. Szuba, et al., Journal of Physics: Conference Series 396 (2012) 022045. http://dx.doi.org/10.1088/1742-6596/ 396/2/022045.

[76] J. Abraham, et al., Astroparticle Physics 33 (2010) 108. http://dx.doi.org/ 10.1016/j.astropartphys.2009.12.005 (arXiv:1002.0366)

[77] P. Abreu, et al., Astroparticle Physics 35 (2012) 591. http://dx.doi.org/10.1016/ j.astropartphys.2011.12.002 (arXiv:1201.2276).

[78] B. Keilhauer, M. Will, European Physical Journal Plus 127 (2012) 96. http: //dx.doi.org/10.1140/epjp/i2012-12096-8 (arXiv:1208.5417).

[79] P. Abreu, et al., Journal of Instrumentation 7 (2012) P09001. http://dx.doi. org/10.1088/1748-0221/7/09/P09001 (10.1088/1748-0221/7/09/P09001).

[80] B. Keilhauer, J. Blümer, R. Engel, H. Klages, M. Risse, Astroparticle Physics 22 (2004) 249. http://dx.doi.org/10.1016/j.astropartphys.2004.08.004 (arXiv: astro-ph/0405048).

[81] F. Arqueros, J.R. Hoerandel, B. Keilhauer, Air Fluorescence Relevant for Cosmic-Ray Detection-Summary of the 5th Fluorescence Workshop, El Escorial, 2007 (Nuclear Instruments and Methods in Physics Research Section A 597 (2008) 1. http://dx.doi.org/10.1016/j.nima.2008.08.056 (arXiv:0807.3760).

[82] B. Keilhauer, J. Bluemer, R. Engel, H. Klages, Nuclear Instruments and Methods in Physics Research Section A 597 (2008) 99. http://dx.doi.org/ 10.1016/j.nima.2008.08.060 (arXiv:0801.4200).
[83] M. Monasor, J. Vazquez, D. Garcia-Pinto, F. Arqueros, Astroparticle Physics 34 (2011) 467. http://dx.doi.org/10.1016/j.astropartphys.2010.10.009 (arXiv:1010.3793)

[84] B. Keilhauer, M. Bohacova, M. Fraga, J. Matthews, N. Sakaki, et al., EPJ Web of Conferences 53 (2013) 01010. http://dx.doi.org/10.1051/epjconf/20135301010 (arXiv:1210.1319).

[85] J. Abraham, et al., Astroparticle Physics 32 (2009) 89. http://dx.doi.org/ 10.1016/j.astropartphys.2009.06.004 (arXiv:0906.5497).

[86] B. Keilhauer, M. Unger, Fluorescence emission induced by extensive air showers in dependence on atmospheric conditions, in: Proceedings of the 31st International Cosmic Ray Conference (ICRC), Lodz, Poland, 2009 (arXiv:0906.5487)

[87] Extensive Air Shower Simulation With Corsika: A User's Guide (Version 74xxx from September 3, 2013) 〈http://web.ikp.kit.edu/corsika/usersguide/ usersguide.pdf (accessed 16.01.15).

[88] P. Abreu, et al., Journal of Instrumentation 8 (2013) P04009. http://dx.doi. org/10.1088/1748-0221/8/04/P04009 (arXiv:1303.5576).

[89] P. Abreu, et al., The Pierre Auger Observatory IV: operation and monitoring in: Proceedings of the 32nd International Cosmic Ray Conference (ICRC), Beijing, China, 2011 (arXiv:1107.4806).

[90] L. Henyey, J. Greenstein, Astrophysical Journal 93 (1941) 70. http://dx.doi. org/10.1086/144246.

[91] S. BenZvi, B. Connolly, J. Matthews, M. Prouza, E. Visbal, et al., Astroparticle Physics 28 (2007) 312. http://dx.doi.org/10.1016/j.astropartphys.2007.06.005 (arXiv:0704.0303)

[92] J. Chirinos, Cloud monitoring at the Pierre Auger Observatory [171], 2013, p. 108 (arXiv:1307.5059)

[93] M. Winnick, Cloud cameras at the Pierre Auger Observatory (Ph.D. thesis) University of Adelaide, 2010, Available online at 〈http://digital.library.ade laide.edu.au/dspace/handle/2440/67198).

[94] P. Abreu, et al., Astroparticle Physics 50-52 (2013) 92. http://dx.doi.org/10. 1016/j.astropartphys.2013.09.004 (arXiv:1310.1641).

[95] V. Rizi, A. Tonachini, M. Iarlori, G. Visconti, European Physical Journal Plus 127 (2012) 92. http://dx.doi.org/10.1140/epjp/i2012-12092-0.

[96] M. Jelinek, M. Prouza, P. Kubanek, R. Hudec, M. Nekola, et al., Astronomy and Astrophysics $454 \quad$ (2006) L119. http://dx.doi.org/10.1051/ 0004-6361:20065092 (arXiv:astro-ph/0606004).

[97] S. BenZvi, et al., New method for atmospheric calibration at the Pierre Auger Observatory using FRAM, a robotic astronomical telescope, in Proceedings of the 30th International Cosmic Ray Conference (ICRC), Merida, Mexico, 2007 (arXiv:0706.1710).

[98] A. Bueno, L. Wiencke, European Physical Journal Plus 127(8) (2012) 98 http://dx.doi.org/10.1140/epjp/i2012-12098-6.

[99] R. Mussa, G. Ciaccio, European Physical Journal Plus) 127 (2012) 94. http://dx doi.org/10.1140/epjp/i2012-12094-X.

[100] M. Micheletti, L. Murruni, M. Debray, M. Rosenbusch, M. Graf, et al., Nuclear Instruments and Methods in Physics Research Section B 288 (2012) 10. http: //dx.doi.org/10.1016/j.nimb.2012.07.022.

[101] M. Micheletti, Aerosol characterization at the Pierre Auger Observatory [171], 2013, p. 104 (arXiv:1307.5059).

[102] L. Porter, J. Earnshaw, E. Tielsch-Cassel, J. Ahlstrom, K. Greisen, Nuclear Instruments and Methods in Physics Research NS-87 (1970) 87. http://dx.doi. org/10.1016/0029-554X(70)90886-4.

[103] M. Giller, G. Wieczorek, A. Kacperczyk, H. Stojek, W. Tkaczyk, Journal of Physics G 30 (2) (2004) 97 (〈http://stacks.iop.org/0954-3899/30/i=2/a=009〉).

[104] F. Nerling, J. Bluemer, R. Engel, M. Risse, Astroparticle Physics 24 (2006) 421. http://dx.doi.org/10.1016/j.astropartphys.2005.09.002 (arXiv:astro-ph/ 0506729).

[105] M. Roberts, Journal of Physics G 31 (11) (2005) 1291 (〈http://stacks.iop.org 0954-3899/31/i=11/a=012 $\rangle$.

[106] J. Pekala, P. Homola, B. Wilczynska, H. Wilczynski, Nuclear Instruments and Methods in Physics Research Section A 605 (2009) 388. http://dx.doi.org/ 10.1016/j.nima.2009.03.244 (arXiv:0904.3230).

[107] M. Giller, A. Smialkowski, Astroparticle Physics 36 (2012) 166. http://dx.doi org/10.1016/j.astropartphys.2012.05.021 (arXiv:1201.4052).

[108] J. Rosado, F. Blanco, F. Arqueros, Astroparticle Physics 55 (2014) 51. http://dx doi.org/10.1016/j.astropartphys.2014.02.003 (arXiv:1401.4310).

[109] M. Ave, et al., Nuclear Instruments and Methods in Physics Research Section A 597 (2008) 41. http://dx.doi.org/10.1016/j.nima.2008.08.052.

[110] M. Ave, et al., Astroparticle Physics 42 (2013) 90. http://dx.doi.org/10.1016/j. astropartphys.2012.12.006 (arXiv:1210.6734).

[111] M. Unger, B. Dawson, R. Engel, F. Schussler, R. Ulrich, Nuclear Instruments and Methods in Physics Research Section A 588 (2008) 433. http://dx.doi.org/ 10.1016/j.nima.2008.01.100 (arXiv:0801.4309).

[112] D. Gora, R. Engel, D. Heck, P. Homola, H. Klages, et al., Astroparticle Physics 24 (2006) 484. http://dx.doi.org/10.1016/j.astropartphys.2005.09.007 (arXiv: astro-ph/0505371).

[113] B. Dawson, M. Giller, G. Wieczorek, Influence of the scattered Cherenkov light on the width of shower images as measured in the EAS fluorescence experiments, in: Proceedings of the 30th International Cosmic Ray Conference, Mexico-City, Mexico, vol. 4, 2007, p. 401. 〈http://indico.nucleares. unam.mx/contributionDisplay.py? contribId $=651 \&$ confld $=4\rangle$.

[114] T. Gaisser, A. Hillas, Reliability of the method of constant intensity cuts for reconstructing the average development of vertical showers, in: Proceedings of the 15th International Cosmic Ray Conference, Plovdiv, Bulgaria, vol. 8, 1977, p. 353. 
[115] M. Tueros, Estimate of the non-calorimetric energy of showers observed with the fluorescence and surface detectors of the Pierre Auger Observatory [171] 2013, p. 11 (arXiv:1307.5059)

[116] D. Veberič, Estimation of the total signals in saturated stations of the Pierre Auger Observatory [171], 2013, p. 23 (arXiv:1307.5059).

[117] K. Kamata, J. Nishimura, Progress of Theoretical Physics Supplement 6 (1958) 93. http://dx.doi.org/10.1143/PTPS.6.93.

[118] K. Greisen, Progress in Cosmic Ray Physics 3 (1956) 1 〈https://archive.org details/progessincosmic031401 mbp >.

[119] D. Newton, J. Knapp, A.A. Watson, Astroparticle Physics 26 (2007) 414. http: //dx.doi.org/10.1016/j.astropartphys.2006.08.003 (arXiv:astro-ph/0608118).

120] M. Ave, Reconstruction accuracy of the surface detector array of the Pierre Auger Observatory, in: Proceedings of the 30th International Cosmic Ray Conference (ICRC), Merida, Mexico, 2007 (arXiv:0709.2125).

[121] C. Bonifazi, A. Letessier-Selvon, E. Santos, Astroparticle Physics 28 (2008) 523. http://dx.doi.org/10.1016/j.astropartphys.2007.09.007 (arXiv:0705.1856).

[122] R. Pesce, Energy calibration of data recorded with the surface detectors of the Pierre Auger Observatory: an update [172], 2011, p. 13 (arXiv:1107.4809)

[123] H. Dembinski, The cosmic ray spectrum above $4 \times 10^{18} \mathrm{eV}$ as measured with inclined showers recorded at the Pierre Auger Observatory [172], 2011, p. 5 (arXiv:1107.4809).

[124] V. Verzi, The energy scale of the Pierre Auger Observatory [171], 2013, p. 7 (arXiv:1307.5059).

[125] H. Tokuno, Y. Tameda, M. Takeda, K. Kadota, D. Ikeda, et al., Nuclear Instruments and Methods in Physics Research Section A 676 (2012) 54 http://dx.doi.org/10.1016/j.nima.2012.02.044 (arXiv:1201.0002).

[126] T. Abu-Zayyad, et al., Pierre Auger Observatory and Telescope Array: Joint Contributions to the 33rd International Cosmic Ray Conference (ICRC), 2013 (arXiv:1310.0647)

[127] I. Valino, A measurement of the muon number in showers using inclined events recorded at the Pierre Auger Observatory [171], 2013, p. 44 (arXiv:1307.5059)

[128] A. Aab, et al., Journal of Cosmology and Astroparticle Physics 1408 (08) (2014) 019. http://dx.doi.org/10.1088/1475-7516/2014/08/019 (arXiv:1407.3214).

[129] I. Mariș, The AMIGA infill detector of the Pierre Auger Observatory: performance and first data [172], 2011, p. 9 (arXiv:1107.4809)

[130] D. Ravignani, Measurement of the energy spectrum of cosmic rays above $3 \times$ $10^{17} \mathrm{eV}$ using the AMIGA $750 \mathrm{~m}$ surface detector array of the Pierre Auger Observatory [171], 2013, p. 4 (arXiv:1307.5059).

[131] H.-J. Mathes, The HEAT telescopes of the Pierre Auger Observatory. Status and first data [173], 2011, p. 1 (arXiv:1107.4807).

[132] F. Suarez, The AMIGA muon detectors of the Pierre Auger Observatory: overview and status [171], 2013, p. 4 (arXiv:1307.5059).

[133] F. Sanchez, The AMIGA detector of the Pierre Auger Observatory: an overview [173], 2011, p. 5 (arXiv:1107.4807).

[134] M. Platino, M. Hampel, A. Almela, A. Krieger, D. Gorbena, et al., Journal of Instrumentation 6 (2011) P06006. http://dx.doi.org/10.1088/1748-0221/6/06/ P06006.

[135] M. Nagano, M. Teshima, Y. Matsubara, H. Dai, T. Hara, et al., Journal of Physics G 18 (1992) 423. http://dx.doi.org/10.1088/0954-3899/18/2/022.

[136] S. Maldera, Measuring the accuracy of the AMIGA muon counters at the Pierre Auger Observatory [171], 2013, p. 4 (arXiv:1307.5059).

[137] O. Wainberg, et al., Journal of Instrumentation 9 (2014) T04003. http://dx.doi. org/10.1088/1748-0221/9/04/T04003.

[138] A. Supanitsky, A. Etchegoyen, G. Medina-Tanco, I. Allekotte, M.G. Berisso, et al., Astroparticle Physics 29 (2008) 461. http://dx.doi.org/10.1016/j.astropartphys.2008.05.003 (arXiv:0804.1068)

[139] B. Wundheiler, The AMIGA muon counters of the Pierre Auger Observatory: performance and first data [173], 2011, p. 9 (arXiv:1107.4807)

[140] S. Dasso, H. Asorey, Advances in Space Research 49 (2012) 1563. http://dx doi.org/10.1016/j.asr.2011.12.028 (arXiv:1204.6196).

[141] P. Abreu, et al., Journal of Instrumentation 6 (2011) P01003. http://dx.doi.org/ 10.1088/1748-0221/6/01/P01003.

142] Public Event Explorer 〈http://auger.colostate.edu/ED/〉 (accessed 16.01.15).

[143] U. Inan, T. Bell, J. Rodriguez, Geophysical Research Letters 18 (1991) 705. http: //dx.doi.org/10.1029/91GL00364.

[144] W. Boeck, O. Vaughan, R. Blakeslee, B. Vonnegut, M. Brook, Geophysical Research Letters 19 (1992) 99.
[145] H. Fukunishi, Y. Takahashi, M. Kubota, K. Sakanoi, U. Inan, W. Lyons, Geophysical Research Letters 23 (1996) 2157.

[146] U.S. Inan, C. Barrington-Leigh, S. Hansen, V. Glukhov, T. Bell, R. Rairden, Geophysical Research Letters 24 (1997) 583.

[147] R. Newsome, U.S. Inan, Journal of Geophysical Research 115 (2010) A00E41. http://dx.doi.org/10.1029/2009JA014834.

[148] A. Chen, et al., Journal of Geophysical Research 113 (2008) A08306. http://dx. doi.org/10.1029/2008JA013101.

[149] A. Tonachini, Observation of Elves at the Pierre Auger Observatory [171], 2013, p. 112 (arXiv:1307.5059).

[150] S.F. Abarca, K.L. Corbosiero, T.J. Galarneau Jr., Journal of Geophysical Research 115 (2010) D18206. http://dx.doi.org/10.1029/2009JD013411.

[151] Visual Physics Analysis-VISPA 〈http://vispa.physik.rwth-aachen.de/ $\rangle$ (accessed 16.01.15).

[152] F.D. Kahn, I. Lerche, Proceedings of the Royal Society of London A: Mathematical, Physical and Engineering Sciences 289 (1417) (1966) 206. http://dx. doi.org/10.1098/rspa.1966.0007.

[153] G. Askaryan, Soviet Physics JETP 14 (1962) 441.

[154] S. Buitink, et al., Shower X_max determination based on LOFAR radio measurements, in: Proceedings of the 33rd International Cosmic Ray Conference (ICRC), 2013, Rio de Janeiro, Brazil.

[155] H. Allan, Progress in Elementary Particles and Cosmic Ray Physics 10 (1971)

[156] D.J. Fegan, Nuclear Instruments and Methods in Physics Research Section A 662 (2012) S2. http://dx.doi.org/10.1016/j.nima.2010.10.129 (arXiv:1104. 2403).

[157] H. Falcke, et al., Nature 435 (2005) 313. http://dx.doi.org/10.1038/nature03614 (arXiv:astro-ph/0505383).

[158] D. Ardouin, A. Belletoile, D. Charrier, R. Dallier, L. Denis, et al., Astroparticle Physics 26 (2006) 341. http://dx.doi.org/10.1016/j.astropartphys.2006.07.002 (arXiv:astro-ph/0608550).

[159] P. Schellart, A. Nelles, S. Buitink, A. Corstanje, J. Enriquez, et al., Astronomy and Astrophysics 560 (2013) A98. http://dx.doi.org/10.1051/0004-6361/ 201322683 (arXiv:1311.1399).

[160] B. Revenu, Autonomous detection and analysis of radio emission from air showers at the Pierre Auger Observatory [173], 2011, p. 17 (arXiv:1107.4807).

[161] J. Kelley, AERA: the Auger Engineering Radio Array [173], 2011, p. 13 (arXiv:1107.4807)

[162] P. Abreu, et al., Journal of Instrumentation 7 (2012) P11023. http://dx.doi.org/ 10.1088/1748-0221/7/11/P11023 (arXiv:1211.0572).

[163] A. Aab, et al., Physical Review D 89 (2014) 052002. http://dx.doi.org/10.1103/ PhysRevD.89.052002 (arXiv:1402.3677).

[164] P. Schellart, S. Buitink, A. Corstanje, J. Enriquez, H. Falcke, et al., Journal of Cosmology and Astroparticle Physics 1410 (10) (2014) 014. http://dx.doi.org/ 10.1088/1475-7516/2014/10/014 (arXiv:1406.1355).

[165] P. Abreu, et al., Journal of Instrumentation 7 (2012) P10011. http://dx.doi.org/ 10.1088/1748-0221/7/10/P10011 (arXiv:1209.3840).

[166] P. Gorham, N. Lehtinen, G. Varner, J. Beatty, A. Connolly, et al., Physical Review D 78 (2008) 032007. http://dx.doi.org/10.1103/PhysRevD.78.032007 (arxiv:hepth/0511259).

[167] P. Allison, Microwave detection of cosmic ray showers at the Pierre Auger Observatory [173], p. 2011, 25 (arXiv:1107.4807)

[168] P. Facal San Luis, EPJ Web of Conferences 53 (2013) 08009. http://dx.doi.org/ 10.1051/epjconf/20135308009.

[169] J. Alvarez-Muñiz, E. Amaral Soares, M. Bogdan, M. Boháčová, et al., Nuclear Instruments and Methods in Physics Research Section A 719 (2013) 70. http: //dx.doi.org/10.1016/j.nima.2013.03.030 (arXiv:1208.2734).

[170] J. Alvarez-Muñiz, J. Berlin, J. Bogdan, J. Boháčová, J. Bonifazi, et al., Physical Review D 86 (2012) 051104. http://dx.doi.org/10.1103/PhysRevD.86.051104 (arXiv:1205.5785).

[171] A. Aab, et al., The Pierre Auger Observatory: Contributions to the 33rd International Cosmic Ray Conference (ICRC), Rio de Janeiro, Brazil, 2013 (arXiv:1307.5059)

[172] P. Abreu, et al., The Pierre Auger Observatory I: The cosmic ray energy spectrum and related measurements, in: Proceedings of the 32nd International Cosmic Ray Conference (ICRC), Beijing, China, 2011 (arXiv:1107.4809).

[173] P. Abreu, et al., The Pierre Auger Observatory V: Enhancements, in: Proceedings of the 32nd International Cosmic Ray Conference (ICRC), Beijing, China, 2011 (arXiv:1107.4807). 